\title{
FUNCTIONAL AND ROBUST HUMAN- MACHINE INTERFACE FOR ROBOTIC-ASSISTED THERAPY OF THE SHOULDER AFTER STROKE
}

\author{
Dissertation \\ zur Erlangung des humanwissenschaftlichen Doktorgrades \\ in der Medizin \\ der Georg-August-Universität Göttingen
}

Vorgelegt von

Dipl.-Ing.

Liliana Patricia Paredes Calderón

aus Cúcuta, Kolumbien

Göttingen, 2016 
Betreuungsausschuss

Doktorvater:

Prof. Dr. Dr. Dario Farina. Professor and chair of Neurorehabilitation Engineering, Imperial College London, England (current position since October 2016). Former director of the Institute of Neurorehabilitation Systems, Universitätsmedizin Göttingen, Germany

Gutachter:

Prof. Dr. med. Walter Paulus. Director of Clinical Neurophysiology, Universitätsmedizin Göttingen, Germany

Gutachter:

Prof. Dr. Monica Reggiani. Assistant professor Department of Management and Engineering of the University of Padua, Italy

\section{Weitere Mitglieder der Prüfungskommission}

Prof. Dr. Silke Schicktanz. Professor at the Department of Medical Ethics and History of Medicine, Universitätsmedizin Göttingen, Germany

Prof. Dr. Tim Beißbarth. Director of Statistical Bioinformatics at the Institute of Medical Statistics, Universitätsmedizin Göttingen, Germany

Prof. Dr. Eberhard Fuchs. Emeritus/Senior professor, German Primate Center, Göttingen, Germany

Prof. Dr. Wolfgang Himmel. Professor at the Department of General Practice, Universitätsmedizin Göttingen, Germany 


\section{Preface}

This thesis presents the results of my work at Otto Bock HealthCare GmbH Duderstadt and the Fondazione Ospedale San Camillo Venedig-Lido, within the MYOSENS project funded by the European Commission's Marie-Curie actions, Industrial Academia Partnerships and Pathways Program (IAPP). Grant No. 286208. Project duration: April $1^{\text {st }} 2012$ - April 1st.2016.

All studies involving human subjects were approved by the Institutional Review Board of Fondazione Ospedale San Camillo and informed written consent was obtained by all participants at the time of enrolment.

All sections of this thesis are original and written by me. Only sections 3.1 and 3.2 have been adapted from one manuscript I submitted to BioMed Central for consideration [1] and one published conference paper in IEEE [2], respectively. All graphics in this manuscript are original and created solely by myself.

Hereby, I declare that I have written this thesis independently and with no other aids and sources than quoted.

Romanshorn $\mathrm{CH}$, August $21^{\text {st }} 2016$

Liliana Patricia Paredes Calderón 


\section{$\underline{\text { Abstract }}$}

The majority of individuals with stroke experience problems with the upper extremity, of which paresis is most common. The use of robotics in upper limb therapy is increasingly popular, as it can deliver intensive and functional arm rehabilitation. This thesis describes the development of a functional and robust myoelectric control interface for the pneumatic shoulder RehaARM robot $^{1}$, using electromyographic biofeedback, and the design of a therapeutic approach for shoulder treatment after stroke with this technology. The therapeutic approach has been evaluated in a phase II clinical trial ${ }^{2}$.

The developed interface goes beyond the state-of-the-art technologies for upper limb therapy in clinical research because it is capable of online myoelectric control of the robot with three degrees of freedom (DoF), supporting shoulder movements. Myoelectric control has been applied only in systems providing 1-DoF movements for the elbow or wrist. The developed RehaARM system surpasses previously developed systems in the number of actuated DoF. More importantly, the developed myoelectric control interface is transferable to other active-assistive robots for upper limb therapy. The thesis also describes novel neurophysiological measurements for evaluating the effectiveness of the treatment with the RehaARM system and presents their correlation with the most commonly used clinical impairment and activity upper limb scales.

\footnotetext{
${ }^{1}$ FerRobotics Compliant Robot Technology GmbH, AT

2 Trial registration: ClinicalTrials.gov, Identifier: NCT02321254 and see section 3.3.2.1
} 
The resulting system has been tested on both healthy volunteers and stroke patients and has been compared with the commercially used torque (force) control. Our experimental studies confirm the benefit of the developed myoelectric control interface for the RehaARM robot. RehaARM with myoelectric control achieved equally desirable effects on muscle activation, namely synergistic activation (muscle recruitment) and modulation of activation levels as torque (force) control.

Results showed that severely, moderately and mildly impaired patients were able to successfully control the RehaARM system with myoelectric control with greater ease than torque control for task-specific shoulder training. Myoelectric control was used by severely impaired patients who have very low residual voluntary forces which are rarely detectable by commercially available robots. For all patients, there was a monotonic increase in the movement completion rate over multiple sessions that showed improvement in voluntary control. The therapeutic approach with the RehaARM myoelectric interface significantly improved both motor control (FMA-UE) and activity (FIM) scores. The difference between effects of robotic and conventional therapy alone - comparable dose and dosage - was not significant. This is in agreement with the evidence found in the scientific literature. Based on the FMA-UE scores, the sample sizes needed to determine the effectiveness of the treatment were similar for conventional therapy $(n=27, p<0.05$; power $=0.8)$ and RehaARM therapy $(n=28, p<0.05$; power $=0.8)$. The results of clinical and neurophysiological assessments in this clinical trial can be used to compare this therapy's effectiveness with future randomized clinical trials of upper limb therapies in terms of effect size of the treatment. Based on the results, the use of the RehaARM robot is continued and its commercial implementation is currently being explored. 


\section{Acknowledgements}

I thank my supervisor Prof. Dr. Dario Farina for the continuous support during my PhD project, grazie mille.

I thank Prof. Dr. med. Walter Paulus for supervising my PhD and giving me his critical and experienced point of view in this neurorehabilitation field, Danke sehr.

I also thank Andrea Turolla and Giorgia Pregnolato for making possible the conclusion of the clinical trial in the Fondazione Ospedale San Camillo and for supporting me during my transformation from engineer to therapist, grazie davvero.

I thank PD Dr. Bernhard Graimann and the Otto Bock Healthcare GmbH for providing me the opportunity to join the MYOSENS project as an early stage researcher.

I thank my ex-colleague Dr. Hubertus Rehbaum for his support. I know that my enrollment in this $\mathrm{PhD}$ program would not have run so smoothly without him. I really learnt a lot from our nerdy technical discussions, muchas gracias!

The interesting discussions with and guidance from all of you have been an invaluable input for my thesis and my future.

I thank my family, and specially my amazing mother Martha Calderón, for providing me with energy and love to get this far. Thanks to her genes and passion for Physiotherapy, I love Neurorehabilitation even more. Gracias!

I thank my amiga Lina María Melo Jiménez for being there for me...en los apuros! Last but not least, I thank my boyfriend Arnoudt Schoppers for accompanying me through the last phase of my PhD and the interesting discussions about Statistics (especially type errors I, II, III and IV), Psychology, logic models, life, fun. Dank je! 


\section{Contents}

Preface

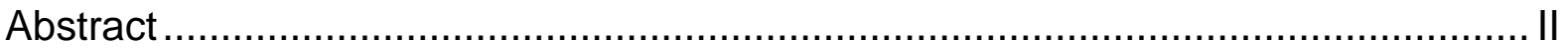

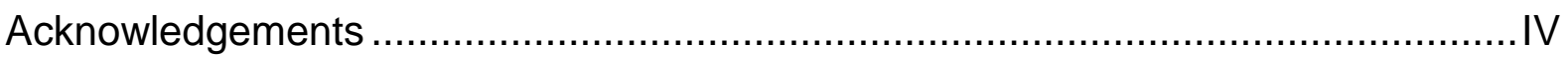

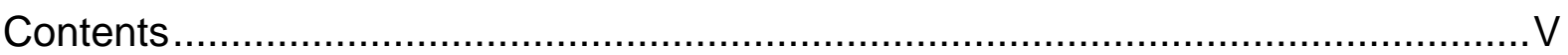

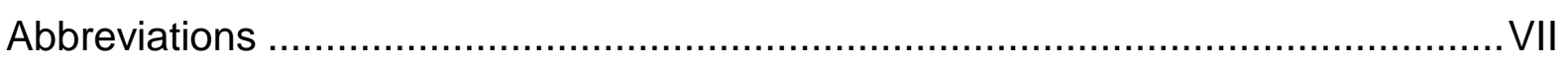

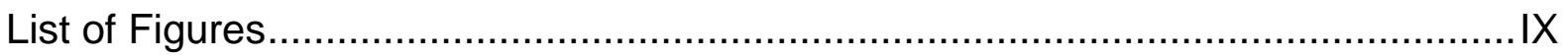

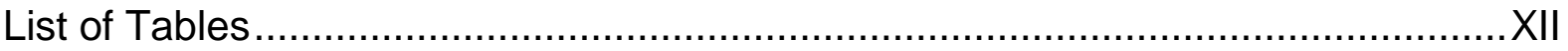

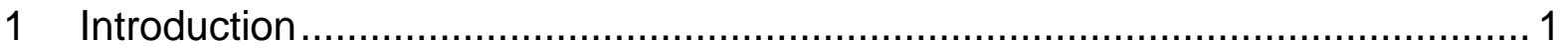

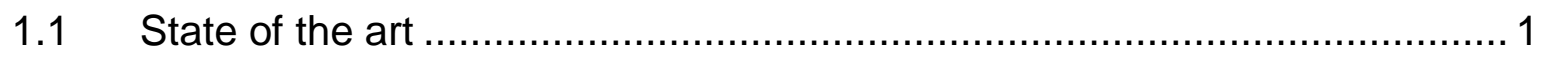

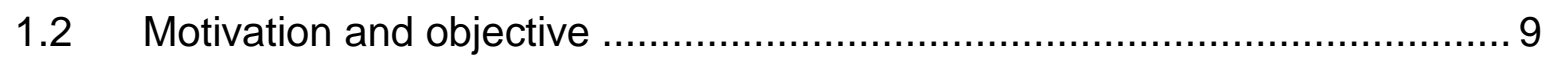

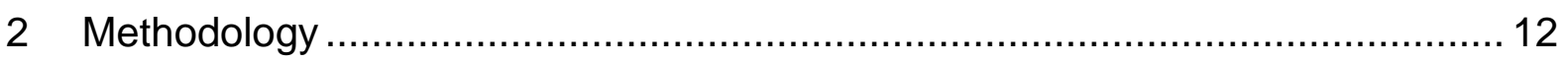

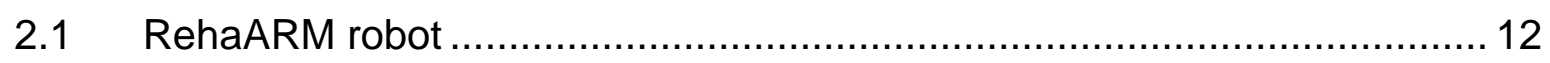

2.2 Online Closed-Loop Command Interface with EMG-Thresholding............. 15

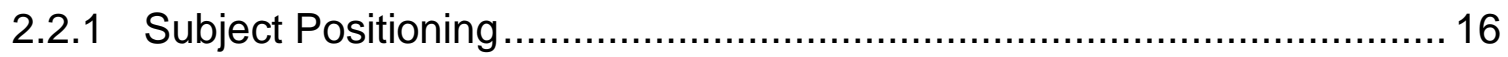

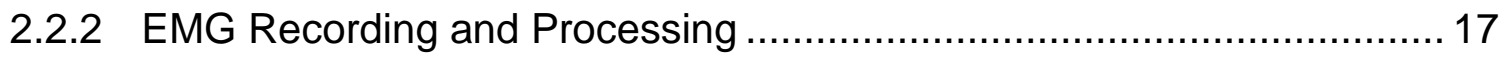

2.2.3 Myoelectric Control with Thresholding ........................................... 18

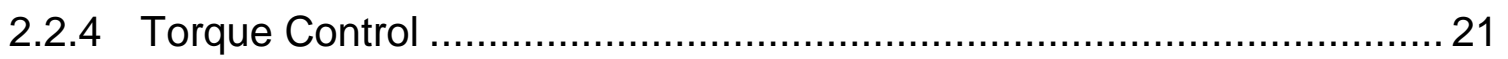

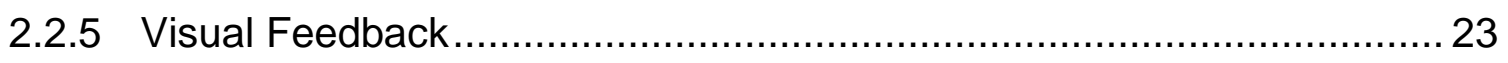

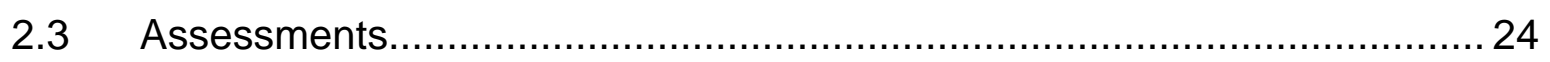

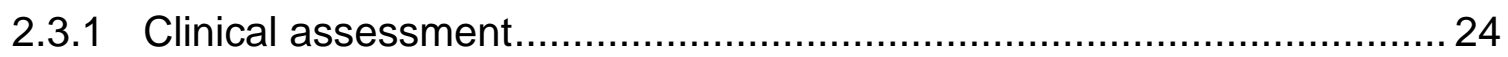

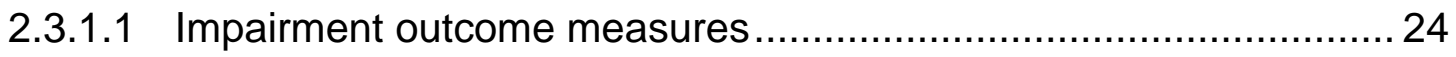

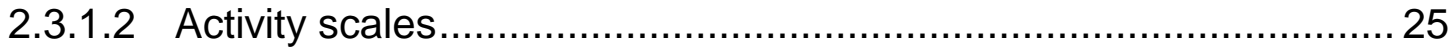

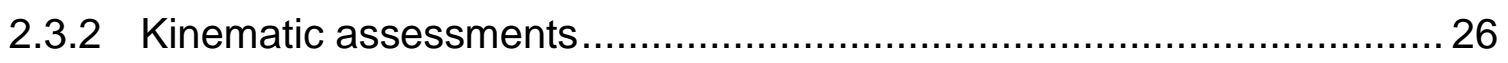

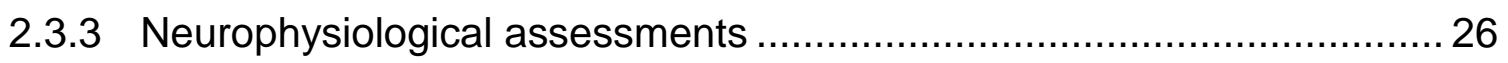

3 Experimental Studies of the Newly Designed Robotic-Assisted Therapy .......... 31

3.1 Study 1: A Novel Pneumatic EMG-driven Robotic System for Shoulder

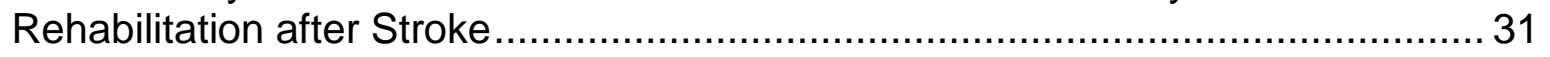

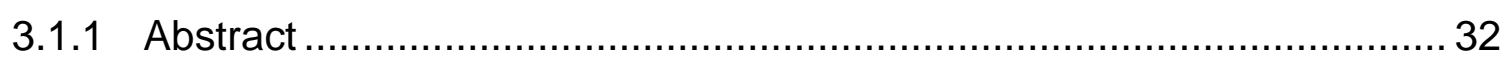

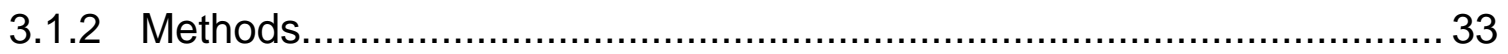

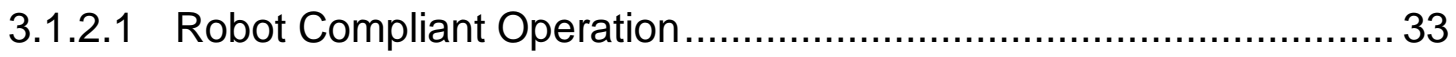

3.1.2.2 Clinical Evaluation Using EMG-Control ....................................... 35

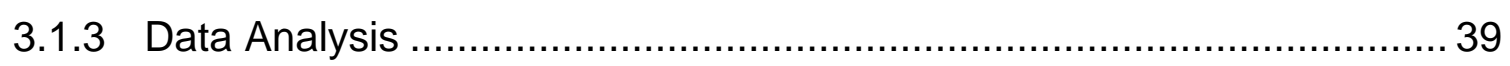

3.1.3.1 Evaluation of the Robot Compliant Operation ............................... 39 
3.1.3.2 Clinical Evaluation with EMG-driven Control ............................. 40

3.1.4 Results .................................................................................... 41

3.1.4.1 Evaluation of the Robot Compliant Operation ............................ 41

3.1.4.2 Clinical Evaluation with EMG-driven Control .............................. 44

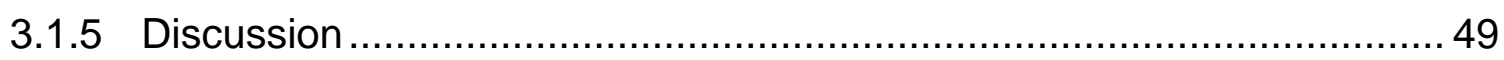

3.2 Study 2: Efficacy of Torque Versus Myocontrol For Active, Robotic-Assisted Rehabilitation Of The Shoulder After Stroke: An Experimental Study Methods .... 54

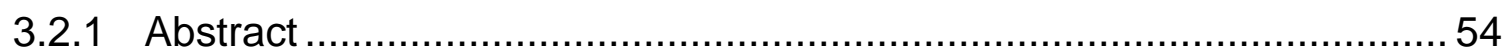

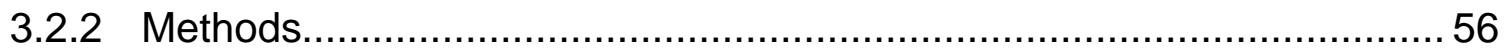

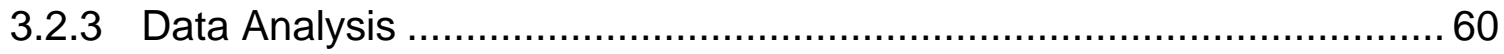

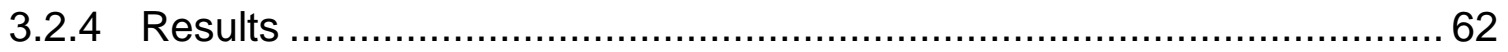

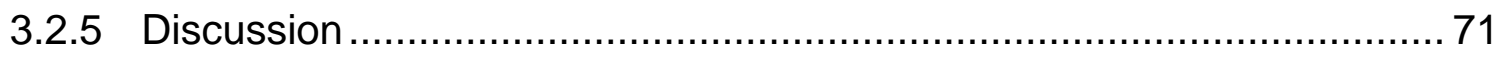

3.3 Study 3: Myoelectric Robot-Assisted Rehabilitation for the Upper Limb after Stroke 73

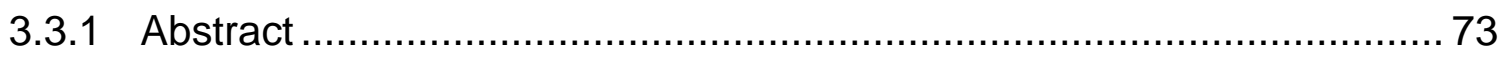

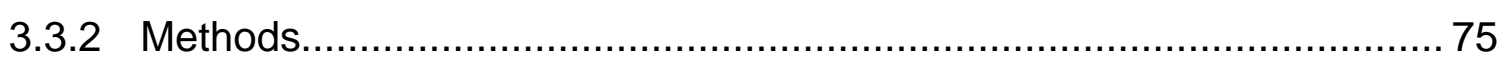

3.3.2.1 Phase II Clinical Study with the RehaARM .............................. 75

3.3.2.2 Comparison of the RehaARM therapy with a Historical Conventional

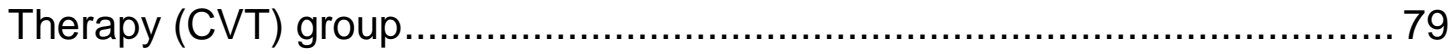

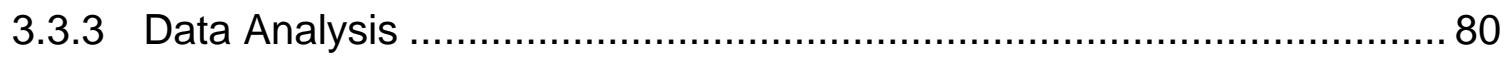

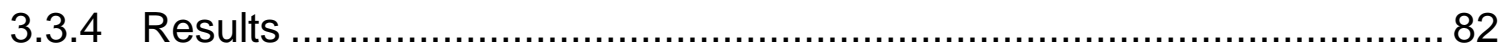

3.3.4.1 Phase II Clinical Study with the RehaARM ............................. 82

3.3.4.2 Comparison of the RehaARM therapy with a Historical Conventional Therapy group (CVT) ................................................................ 88

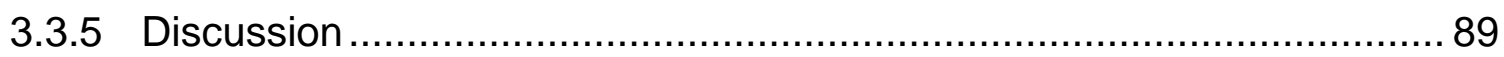

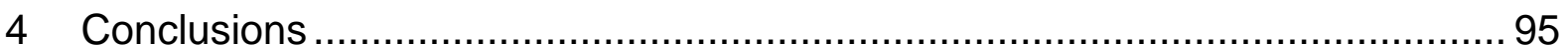

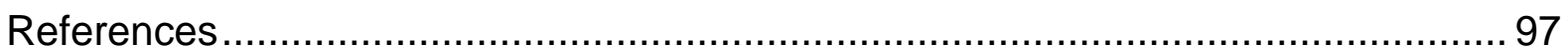

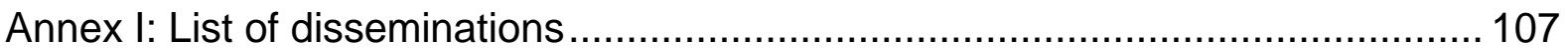

Annex II: Curriculum Vitae................................................................... 108 


\section{$\underline{\text { Abbreviations }}$}

ADLs Activities of daily living

ABD $\quad$ Abduction

ADD Adduction

CIMT Constraint induced movement therapy

CVT Conventional therapy group

CT Computer tomography

DoF $\quad$ Degrees of freedom

EMG Electromyography

EMG-S Similarity between the muscle activation of healthy subjects (median) and the muscle activation of stroke patients when using EMG-driven control

EXT

Extension

FLEX

Flexion

FMA

Fugl-Meyer Assessment of motor recovery after stroke

FMA-UE

Upper extremity section of the Fugl-Meyer Assessment of motor recovery after stroke

FIM

Functional Independence Measurement

ICA Internal carotid artery

$\mathrm{ICH} \quad$ Intracerebral hemmorhage

IE Internal/external rotation

MAS Modified Ashworth Scale

MCA Middle cerebral artery 
MDC

MCID

MRI

Motor-FIM

MVC

NHPT

NMES

NMF

PCA

$\mathrm{PACl}$

$\mathrm{POCl}$

RPS

ROM

$\mathrm{SAH}$

SMI

SRI

$\mathrm{TACl}$

TORQUE-S

UE
Minimum detectable change

Minimally clinically important difference

Magnetic resonance imaging

Motor portion of the Functional Independence Measurement

Maximum voluntary contraction

Nine-Hole Pegboard Test

Neuromuscular electrical stimulation

Non-matrix factorization

Posterior cerebral artery

Partial anterior cerebral Infarct

Posterior cerebral infarct

Reaching Performance Scale

Ranges of motion

Subarachnoid hemmorhage

Somato-sensory integration

Stroke to rehabilitation interval

Total anterior circulation infarct

Similarity between the muscle activation of healthy subjects (median) and the muscle activation of stroke patients when using torque control

Upper extremity 


\section{List of Figures}

Figure 1: Model of the RehaArm robot. a) Right arm exoskeleton and its three rotational axes for DoF1, DoF2 and DoF3, corresponding to horizontal adduction/abduction (HORZ ADD/ABD), adduction/abduction $(A D D / A B D)$ and flexion/extension (FE), and internal/external rotation (IE), respectively (c). The robot arm is connected to a chair (b) on which the subject sits comfortably, entirely supporting the arm on the exoskeleton while executing the movements (d). The surface EMG electrodes are mounted on the shoulder muscles to implement EMG-driven control.

Figure 2: Closed-loop control framework developed for RehaArm. (a) A control PC was connected to an EMG amplifier, the robot and a computer monitor. (b) Three unidirectional TCP/IP connections were used to configure the robot, send the desired position, and receive the sensor data. (c) Visual feedback to the subject displayed on the monitor placed in front of the subject for the single-DoF task (HORZ ADD/ABD) (c1) and for the EIGHT task, a simultaneous combination of two single DoF tasks: HORZ ADD/ABD and FE (c2). The grey shaded area in c1 was displayed in the tasks with EMG-driven control (section 2.2.5). See text for explanation. ... 16

Figure 3: Diagram of the EMG-driven (both switches in position (1)) and torque control (both switches in position (2)) algorithms for the robot's active assistance in the engineering studies 1 and 2 (see sections 3.1.2.2 and 3.2) and the clinical trial (study 3, section 3.3). In myoelectric control, the robot assisted the patient to progressively complete the task if the momentary level of muscle activation (AEMG the respective muscle at the moment $\boldsymbol{t m}$ for the chosen task exceeded the required muscle threshold (ThEMGkm) and the patient followed the desired trajectory. In torque control, the robot assisted the patient to progressively complete the task if the momentary torque level $(\mathbf{T} \boldsymbol{j m})$ at the moment $\boldsymbol{t m}$ for the chosen task (j) exceeded the required task threshold (ThTORQUEj) and the patient followed the desired trajectory.

Figure 4: Forward synergy concept for the control of the shoulder flexion/extension. In (a) from the motor cortex, the low dimensional motor commands (primitives or activation coefficients, P) are sent to the spinal cord (S). In the spinal cord, $\mathrm{P}$ is transformed into the high dimensional muscle activation signals ASEMGi $\boldsymbol{i}=1,2,3$, necessary to execute the intended movement. Finally, the muscle activation is recorded by the multi-channel bipolar surface EMG system. In (b), the model is schematically illustrated. It can be visualized how the recorded EMG signals can be reconstructed by linearly combining several time-invariant muscle synergies $(\boldsymbol{S 1}, \boldsymbol{S} 2)$, each activated by a distinct timedependent coefficient waveform (primitives, $\boldsymbol{P 1}, \boldsymbol{P 2})$. Each of the two synergies $(\boldsymbol{S}$, dimension $3 \times 2)$ weights the coefficients ( $\boldsymbol{P}$, dimension $2 \mathrm{X}$ nSamples) in order to obtain three muscle activations ( $\boldsymbol{A} \boldsymbol{S}$, dimension $3 \mathrm{X}$ nSamples). ' $\mathrm{nSamples'} \mathrm{is} \mathrm{the} \mathrm{number} \mathrm{of} \mathrm{samples} \mathrm{per} \mathrm{task} \mathrm{(nSamples}=$ time*2048 $\mathrm{Hz}$ ), the number of synergies is two, the number of muscles is three and there are two primitives $(\boldsymbol{P 1}$ for shoulder flexion and $\boldsymbol{P 2}$ for shoulder extension). .28

Figure 5: Visual feedback to the subject displayed on the monitor placed in front of the subject for the singleDoF target tracking task (HORZ ADD/ABD) (a) and for the EIGHT target-tracking task, a simultaneous combination of two single DoF tasks: HORZ ADD/ABD and FE (b).

Figure 6: Modulation of EMG activity of triggering muscles during the target-tracking single-DoF by changing task and configuration parameters. Normalized mean value of the RMS of the EMG-signals recorded from (a) Posterior Deltoid of subject 1 during horizontal abduction (HORZ ABD), (b) Pectoralis Major of subject 2 during horizontal adduction (HORZ ADD) and (c) Middle Deltoid of subject 3 during shoulder abduction ( $A B D$ ) across the condition $\mathrm{Ci}, \mathrm{i}=1, \ldots, 6$ of Table II. The terms (soft, stiff) and (slow, fast) are descriptive names for the (minimum, maximum) values in the columns "Maximum torque around the DoF" and "Speed of Movement" in Table II, respectively.

Figure 7: Modulation of EMG activity of pectoralis major and teres major during an EIGHT task when modifying the parameter maximum torque along HORZ ADD/ABD in one representative subject. Linear envelopes of pectoralis major $(a, b)$ and teres major $(c, d)$ in both soft $(a, c)$ and stiff $(b, d)$ mode, respectively. The 
annotation [FE, HORZ ABD/ADD] indicates the maximum torque setting around the FE and HORZ ADD/ABD DoF, respectively.

Figure 8: Activation profile of six shoulder muscles, $\boldsymbol{A E M G 1 , . . . ~ A E M G 6 ~ ( a ) ~ a n d ~ r o b o t ~ p o s i t i o n ~ ( b ) ~ w h e n ~ a ~}$ representative patient with a mild impairment was performing the exercise of internal/external rotation, DoF3 (c) using myoelectric control. The $\boldsymbol{A} \boldsymbol{E} \boldsymbol{M G} \boldsymbol{k}$ were the activation profiles determined and saved online during the session. The external rotation corresponded to a displacement of 46 degrees (from $37.6^{\circ}$ to $-8.4^{\circ}$ ) and the internal rotation corresponded to the reverse movement. The patient moved mostly along DoF3 (task direction), but the movement was not entirely planar, since there were some excursions along DoF1 and DoF2 (approx. $10^{\circ}$ and $2.9^{\circ}$, respectively). The teres major and infraspinatus muscles were the triggering muscles for the internal and the external rotation, respectively. The activity of these muscles was strong enough to drive the robot progressively through the task without the robot's passive assistance. In (b) $R(t)$ is the reference position which the patient should follow and $\mathrm{O}(\mathrm{t})$ is the patient's generated trajectory. . .44

Figure 9: Completion rate (CR) for group A and group B using the EMG-driven control system over consecutive days (black boxplots) and median completion rate (CR) for group A and group B over all days (dashed grey boxplots). The target CR per session for each control modality was 40 repetitions (5 repetitions $x 4$ single-DoF tasks $\times 2$ directions). There was a tendency towards a higher number of repetitions over consecutive days for both groups, but no significant difference was found. Group B executed a significantly higher number of repetitions over all days ( $p<0.0001$, grey asterisks and bar) in comparison to group $\mathrm{A}$.

Figure 10: Activation profiles of six muscles during the execution of a HORZ ADD/ABD for a representative healthy subject (a), a mild patient (b) and a severe-to-moderate patient (c). The threshold values for the triggering muscles corresponded to the $35 \% \mathrm{MVC}$ and the baseline corresponded to the maximum EMG activity at rest. Note the similar threshold values for the representative healthy subject and mild patient. While these values for the representative severe patient were markedly lower, especially for the posterior deltoid. The EMG activity of the pectoralis was similarly strong for all subjects (See also table VI). Note that the axes range for each plot is different, e.g. for the infraspinatus, teres and deltoid muscles of the severe subjects, the range goes from [0-4] $\mathrm{mV}$. . .47

Figure 11: Activation profile of six shoulder muscles, $\boldsymbol{A E M G 1}, \ldots \boldsymbol{A E M G 6}$ (a), torque (b) and robot position (c) when the representative patient with a mild impairment was performing the exercise of internal/external rotation, DoF3 (d) using torque control. The $\boldsymbol{A E \boldsymbol { M } G \boldsymbol { k }}$ were the activation profiles determined and saved online during the session. The external rotation corresponded to a displacement of 46 degrees (from $37.6^{\circ}$ to $-8.4^{\circ}$ ) and the internal rotation corresponded to the reverse movement. The patient moved mostly along DoF3 (task direction), but the movement was not entirely planar, since there were some excursions along DoF1 and DoF2 (approx. $13^{\circ}$ and $2.3^{\circ}$, respectively). The subject activated the robot when the torque value around DoF3 exceeded $1 \mathrm{Nm}$ in the corresponding direction. The patient's residual force was also strong enough to drive the robot progressively through the task without the robot's passive assistance. . .61

Figure 12: Torque (b) when the representative patient with severe-to-moderate impairment (c) and with mild impairment were performing the exercise of internal/external rotation, DoF3 (b, d) using torque control. For the severe-to-moderate patient, the external rotation corresponded to a displacement of 46 degrees (from $6^{\circ}$ to $-40.8^{\circ}$ ) and the internal rotation corresponded to the reverse movement. The subject activated the robot when the torque value around DoF3 exceeded $0.5 \mathrm{Nm}$ for (a) and $1 \mathrm{Nm}$ (b) in the corresponding direction. In (a), the patient's residual force was not strong enough to drive the robot. Therefore, the passive mode was activated by the physiotherapist in order to avoid excessive fatigue. For $(c)$, the patient's residual force was strong enough to drive the robot progressively through the task without the robot's passive assistance. .62

Figure 13: Task completion rate (CR) for group A and group B using the EMG-driven control system over all days (left grey shaded area) and task completion rate (CR) for group $A$ and group $B$ using torque control over all days (right white area). The target CR per session for each control modality was 40 repetitions (5 repetitions $\times 4$ single-DoF tasks $\times 2$ directions). Group B executed a significantly higher number of repetitions over all days using myoelectric and torque control ( $p<0.0001$, asterisks and bar) in comparison to the group $\mathrm{A}$. 
Figure 14: Completion rate (CR) for group A (a) and group B (b) using the EMG-driven (grey-shaded areas) and torque control system (white areas) over consecutive days. The target CR per session for each control modality was 40 repetitions ( 5 repetitions $\times 4$ single-DoF tasks $\times 2$ directions). There was a tendency towards a higher number of repetitions over time for both groups. Group A performed better with torque and myoelectric control at day 1 in comparison to day $4(p<0.05)$. For group $B$, no significant difference was found.

Figure 15: Subjective opinion for group A (light gray boxplots), group B (white boxplots) and healthy subjects (dark grey boxplots) about the ease of use of each control modality after day 1 (left) and session 4(b). 66

Figure 16: Results of the muscle activation similarity over all tasks for group A (light gray boxplots), group B (white boxplots) and all patients (dark grey boxplots). The muscle activation of all tasks was obtained using the NNMF algorithm (see section 2.3.3) from 16 surface EMG channels recorded session 4 for the patient groups and at the only daily session for the healthy volunteers (see section 2.2.2). .. 67

Figure 17: Median synergy set for the healthy subject group $(n=10)$. This is the reference of muscle recruitment for using the robot with myoelectric (upper two rows) and torque control (lower two rows). Healthy subjects recruited muscle patterns very similarly when using myoelectric control and torque control (overall median similarity $=0.98$ of all tasks and control modalities). 68

Figure 18: Synergies for a representative patient from group B (green bars) and the median synergies of healthy subjects (black bars) for all single-DoF tasks and both control modalities EMG-driven control (upper two rows) and torque control (lower two rows). The EMG-S for this subject was 0.8 and the TORQUE-S for this subject was 0.85 . The triceps brachii, lateral and medial head $(\leftarrow)$, was markedly more activated in the HORZ ABD task in comparison to the median activation of the same muscle for the healthy subjects.

Figure 19: Synergies for a representative patient from group A (light blue bars) and the median synergies of the healthy subjects group (black bars) for all single-DoF tasks and both control modalities EMG-driven control (upper two rows) and torque control (lower two rows). The EMG-S for this subject was 0.7 and the TORQUE-S for this subject was 0.69 . The biceps brachii, long head $(\boldsymbol{\nabla})$, was markedly more activated in five out of eight tasks of myoelectric control and in sex out of eight tasks of torque control in comparison to the median activation of the same muscle for the healthy subjects. For the tasks ABD/ADD and FLEX/EXT in torque and myoelectric control, the biceps brachii short head $(\rightarrow)$ and the superior trapezius $(\leftarrow)$ were markedly more activated in comparison to the median activation of the same muscle for the healthy subjects.

.. 71

Figure 20: Raw EMG signals (a) and synergies (b) for the task ABD/ADD in the initial assessment (upper greyshaded area) of a representative patient from the severe-to-moderate group A and raw EMG signals (c) and synergies (d) for the same task in the final assessment (bottom white area) of the same patient. The signals from 15 muscles and the corresponding synergy modules are displayed. The muscle 8 (trapezius) was excluded because of strong artifacts. The raw EMG signals and the synergies show an improvement after treatment. In the final assessment, there was activity in the teres $(\downarrow)$ and medium deltoids $(\nabla)$ in contrast to the initial assessment. The black synergy modules in (b) and (d) represent the median muscle activity of the healthy subjects group and the light blue synergy modules represent the muscle activity of the patient. Note that the scale of the EMG signals in (a) and (c) is the same. .. 86 


\section{List of Tables}

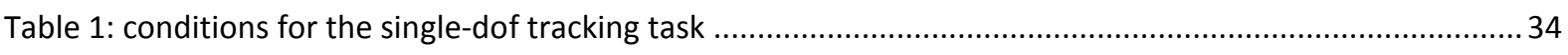

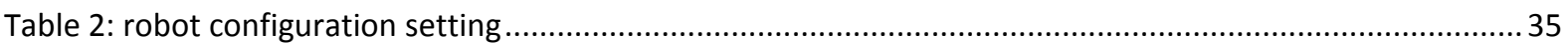

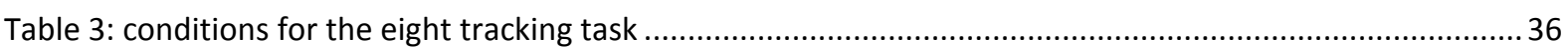

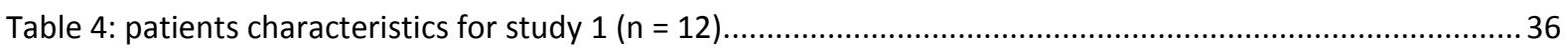

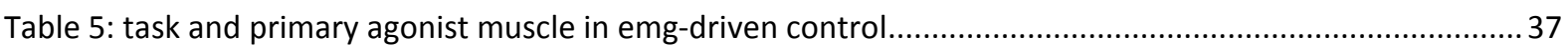

Table 6: maximum values of emg activity of the rotator cuff muscles for each task (median; mean \pm std) ........48

Table 7: maximum values of emg activity of the triggering muscles for each task (median; mean \pm std) ...........48

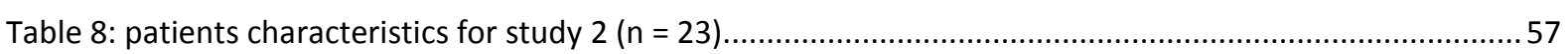

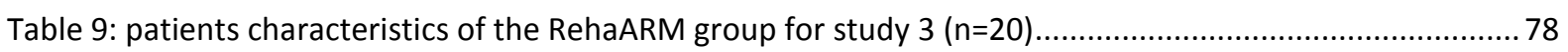

Table 10: effect of therapy on the Fugl-Meyer Upper Extremity Scale (FMA-UE) .........................................82

Table 11: effect of therapy on the Functional Independence Measure (FIM) ............................................. 83

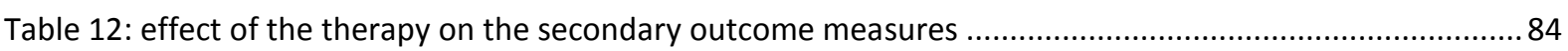

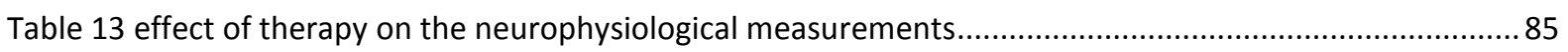

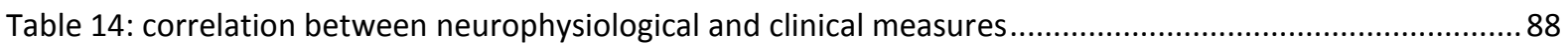

Table 15 effect of therapy on the FMA-UE and FIM scale in the RehaARM and CVT group ............................89 


\section{Introduction}

\subsection{State of the art}

Stroke is worldwide a leading cause of disability [3]. The corresponding incidence rates $^{3}$ standardized to the world population are $71(95 \% \mathrm{Cl} 55$ to 88$)$ for ischemic stroke (cerebral infarction), 16 (95\% $\mathrm{Cl} 8$ to 23$)$ for intracerebral hemorrhage, 6 (95\% $\mathrm{Cl} 1$ to 11$)$ for subarachnoid hemorrhage, and $7(95 \% \mathrm{Cl} 2$ to 12$)$ for undetermined stroke [4].

Most stroke individuals survive, albeit often with severe impairments, such as sensory and motor hemiparesis, spasticity, and lack of coordination in the muscle activation of the contralateral side of the body. The most common post-stroke problem is paresis of the upper extremities. Upper limb paresis is found in more than two-thirds of all patients at hospital admission [5], [6]. After a six month period, while performing activities of daily living (ADLs) the upper limb remains non-functional in up to $66 \%$ of all stroke patients and only $5 \%$ to $20 \%$ demonstrate complete functional recovery [7]. Therefore, the most disabling of all residual impairments is to remain without function in ADLs. This can lead to loss of independence in ADLs and of important occupational activities (e.g. work, driving). Furthermore, it can even result in institutionalization and the development of neuropsychological disorders like depression and anxiety [8].

Effective post-stroke upper limb rehabilitation has not yet been reached. In light of this limited success of traditional rehabilitation programs in recovering upper limb

\footnotetext{
${ }^{3}$ Rates per 100.000 population per year for first-ever-in-a-life-time stroke. Age and sex adjusted to the world population of Segi. Statistics based on a study done in Melbourne, Australia (1996-1997)
} 
function after stroke, research has focused on innovative motor recovery therapeutic approaches. It has been shown that for stroke survivors highly repetitive, taskspecific upper limb training facilitates cortical plasticity in the brain and is effective for improving motor abilities and functional activity performance of the upper limb [9][12]. In other words, task-specific training or functional task practice is based on the premise that practice of an action results in enhanced performance of that action by focusing on learning or relearning motor skills (neuroplasticity) [13], [14]. A number of techniques have been proposed to facilitate the delivery of or include task-specific training, including constraint-induced movement therapy (CIMT), neuromuscular electrical stimulation (NMES), virtual reality and robot-aided rehabilitation [8].

CIMT can be beneficial for eligible stroke survivors [8]. According to an in 2015 updated Cochrane review, CIMT appeared to be more effective at improving arm movement than active physiotherapy treatments or no treatment. However, the quality of the evidence is limited by the small numbers of study participants and poor reporting of study characteristics [15]. NMES can be effective for individuals with minimal voluntary movement within the first few months after stroke or for individuals with shoulder subluxation. NMES can be used in combination with task-specific training, especially for the wrist and hand muscles [8]. Virtual reality and gaming are reasonable treatments to engage individuals with stroke and increase the amount of upper limb movement practice because it can be enjoyable for patients. However, virtual reality without arm support can be used solely with patients with moderate to mild impairment who have some active control of the upper limb and can overcome gravity by lifting the arm. An in 2015 updated Cochrane review found evidence that the use of virtual reality and interactive video gaming may be effective in improving upper limb function and ADL function when used in combination with conventional 
therapy (to increase overall therapy time) or compared with the same dose of conventional therapy. These results should be interpreted cautiously as the studies had small numbers of participants [16].

Other therapeutic modalities like mental imagery and strengthening exercises are also reasonable to consider in combination with functional task practice. The following therapeutic modalities have been the target of multiple studies, but have not yet shown consistent effectiveness in post-stroke upper limb motor recovery. These modalities are: noninvasive brain stimulation (transcranial magnetic stimulation, TMS, or transcranial direct current stimulation, tDCS), somato-sensory stimulation combined with upper limb exercise therapy, and interventions focusing on motor apraxia and manual therapy approaches such as stretching, passive exercise, and mobilization. These modalities are standard treatments for more severely impaired individuals used to prevent contractures and to manage spasticity [8].

Robotic devices are particularly interesting because they can provide exercise therapies in accurate, reproducible and high dosages. It can help patients regain their arm function by supporting the patients' movement with fun exercise games in virtual reality that motivate them towards a more active effort and greater involvement. Robotic therapy can be an effective treatment for providing more intensive therapy to individuals with moderate to severe upper limb paresis. Numerous studies have shown its positive effect. A Cochrane review updated in 2015 found that upper limb robotic therapy provided benefits with regard to ADLs and arm function. However, studies comparing dose-matched exercise between robotic and conventional therapy have shown minimal or no differences in the efficacy of these two treatments [5], [8]. Robotic-assisted therapy will not give better results than human delivery movement therapy if all variables are matched [17]. 
Robotic therapy in combination with conventional therapy is becoming increasingly common for treatment of the upper limb. Robotic devices have the potential to complement traditional neurological rehabilitation requiring greater investments of resources and time. Recovery after stroke varies markedly. Certain people can restore their upper limb function relatively quickly; for others recovery can take a long time or can even be a lifelong process [18]. There is a lack of professionals and the costs for adequately covering all patients who need inpatient and outpatient rehabilitation are high. The higher intensive training with robotic devices can be justified considering its cost-effectiveness.

Therapy is generally provided on one to one ratio of therapist to patient. Roboticassisted therapy may potentially facilitate therapy with a lower ratio, without negatively affecting efficacy. Clinical research should employ well-designed randomized clinical trials to test whether robotic-assisted therapy - with a lower than one ratio of therapists to patients - in combination with one-on-one conventional therapy has a higher efficacy and greater cost-effectiveness compared to one-onone conventional therapy only. This could be one therapist providing therapy to two ${ }^{4}$ or more patients [19], or for example, two therapists per three or more patients. This is already happening in rehabilitation clinics ${ }^{4,5}$ and will become more popular in a near future. This may contribute to reducing healthcare costs, or to increasing the amount of therapy provided. Future research should examine the feasibility and impact on efficacy of such settings taking on account impairment level and patient's state.

\footnotetext{
${ }^{4}$ For example, at St. Mauritius Therapieklinik, Meerbusch, Deutschland one therapist treats two patients (1:2 ratio) with similar impairments

${ }^{5}$ For example, Loius Stokes Cleveland Department of Veteran Affairs (VA) Medical Center, Medical Research, Cleveland, $\mathrm{OH}$
} 
In recent years, the compliant behavior of rehabilitation robots has become a relevant factor in their further development in order to ensure safe interaction with the patient, emulate the properties of human limbs, and provide human-like contact [20]. The compliant operation can be implemented in two ways: it can be actively simulated using electric drives [21] (e.g. impedance control) or it can be an inherent feature of the robot's structural/mechanical design, as for example, when using pneumatic actuators [22].

There are several robots driven by electric motors that implement active compliance for upper limbs training and/or gravity compensation. For example, InMotion ARM ${ }^{\mathrm{TM}}$ (clinical version of the MIT-Manus) is a commercial 2-DoF robot manipulator that assists shoulder and elbow movement by guiding the patient's hand in the horizontal plane [23]. The Assisted rehabilitation and measurement guide (ARM-GUIDE) supports shoulder and elbow movements in different directions. The user's hand is moved along a linear rail similar to a trombone slide [24]. The Mirror Image Movement Enabler (MIME) is a 6-DoF robot manipulator for the training of the elbow and shoulder [25]. The ARMin, and its commercially available version ArmeoPower, support the motion of shoulder, elbow, and wrist joints (6 DoF), covering a full 3-D workspace [26].

Pneumatic robots are increasing in popularity because of the high power-to-weight ratio, low cost and direct drive capabilities of pneumatic actuators [27]. RUPERT is a 5-DoF robot for assisted shoulder elevation, humeral external rotation, elbow extension, forearm supination and wrist/hand extension [28]. The Salford rehabilitation exoskeleton (SRE) is a wearable 7-DoF gravity compensating exoskeleton, supporting the shoulder (3 DoF), elbow (2 DoF) and wrist movements (2 DoF) [20]. The iPAM (Intelligent Pneumatic Arm Movement) consists of two 
identical robot arms: the distal orthosis controls the movement of the patient's forearm, while the proximal orthosis - attached at the mid-point of the upper arm controls the proximal arm movement. With a total of six active DoF, it can support the movements of the shoulder (5 DoF) and elbow (1 DoF) joints, providing assistance during, for example, reach-retrieve, pick-and-place and hand-to-mouth movements [27]. The PNEU-WREX is a 4-DoF, gravity compensating robot assisting elbow flexion/extension, shoulder horizontal abduction/adduction, flexion/extension, and forward-backward translation. The system monitors the patient's behavior and constructs a real-time computer model of the patient's level of impairment. The model is then used to provide feedforward assistance using a compliant position controller [29].

Commercially available rehabilitation robots for treatment of the upper limb use control schemes like force, torque and impedance control. The most common control paradigm is the impedance control which has been proven adequate for high-ratio transmitting systems. In this scheme, the robot reads information from the humanmachine interaction through contact forces and uses it to support the limb in the rehabilitation tasks. It has been applied successfully in numerous robot applications that involve human-motor interaction. This control scheme combined with complex tasks in a 3-D space has proven to be successful for use with less severely impaired patients who have some degree of control to break out of their stroke pattern and some residual force to interact with the robot. The stroke patterns for the arm are: i) the arm flexion pattern that is the most common among stroke survivors - arm resting against the body, elbow flexed with forearm across the abdomen and hand closed - or ii) the arm extension pattern - elbow, wrist and fingers rigidly straight. 
The most well-studied robotic devices (e.g. ARMin, MIME, MIT-MANUS, [30]) use impedance control or measure residual volitional force to activate the robot [23], [31]. From my experience on the field of robotic-assisted technologies for upper limb therapy, the drawback of some commercially available active-assistive robots is the lack of sensitivity and transparency, and therefore, the responsiveness of these systems (force sensors cannot read the intended movement) to the scarce residual force of the paretic upper limb of stroke survivors. The system's sensitivity is the minimum magnitude of an input signal required to produce a specified output signal having a specified signal-to-noise ratio, or other specified criteria. Transparency refers to apparent robot dynamics that the user feels in "free space" motion, when the user moves the robot. The user should not feel these apparent dynamics. Forces that need to be overcome when moving a robot are inertia, gravity, friction, and Coriolis and centrifugal forces. The sensitivity and transparency of the system are determined by the sensors (e.g. force sensors in force/torque control) and the control. A lightweight construction and/or compliant actuation reduce these forces, but this reduction is limited [32]. The users, especially the more severely impaired patients, feel this residual seeming inertia, cannot often overcome it and cannot activate the robot. This limitation applies to closed-loop impedance and force/torque controlled electrical robots [32], [33] and pneumatic robots. Consequently, severely impaired patients are often assigned to conventional therapy only because they cannot activate the robot. High sensitivity and transparency is a prerequisite to keeping the patient engaged in the task and permitting him to observe his successful and unsuccessful attempts at moving.

Another drawback is the high complexity of tasks. The tasks and games are often too complex for the lacking sensitivity and transparency of the robot, and the few 
available control signals from the paretic upper limb. Even though some systems can be sensitive to detecting the movement of some joints, the patient (particularly those more severely impaired) is often overwhelmed and cannot execute complex tasks involving the activation of more than one DoF.

Finally, there is a mismatch between the few control signals obtained from the subject's attempt of movement and the task complexity. Therefore, some of the systems arbitrarily decide the path of a complex task based on few control signals while there are usually multiple paths for executing a task. Especially for gaming in a 3-D space, those systems' schemes attached to a predefined trajectory are impeding the subject's freedom of movement. As the complexity of the task increases, the appropriateness of a predefined trajectory decreases. As a result, the subject neither understands the task nor acts as the initiator of his movements. This can be confusing for patients (especially for more severely impaired patients) given their cognitive disabilities after stroke. And therefore learning is inhibited and frustration arises.

The only extensive study on the feasibility of using the 7-DoF ARMin robot with patients with severe-to-moderate impairments has been published in Lancet Neurology [26]. The results of this multicenter, randomized clinical trial showed the practicability of the ARMin robot for upper limb therapy and agreed with previous available evidence: upper limb training with a robotic system is safe and improves motor function and abilities of ADLs [34]. However, force sensors and closed-loop force/impedance control may not always be enough to detect and use the residual voluntary force of severely impaired patients for controlling the robot. Other techniques, like myoelectric control, can complement the readings from the force 
sensors and the control system to help better interpret and use the subject's intention of movement [18].

There are only few active robotic rehabilitation systems integrating EMG signals into the control loop. In two studies [35], [36], EMG signals were used to control exoskeleton robots, by estimating the joint torque generated by the subject according to the EMG signals, and then computing the required assistive torque. In another study [30], surface EMG was used to detect the intention of the user to move the elbow and then, when necessary, provide assistance using a powered elbow orthosis (Myomo, Inc., Boston, MA, USA). Other researchers [37], proposed an active assistive robot with myoelectric control in a closed loop for elbow rehabilitation using a tracking task, and a similar concept was later applied to the wrist [38]. They demonstrated better clinical outcomes for the myoelectric (also EMG-driven) robot of the wrist compared to passive (open loop) robot training. Single DoF exercises at the elbow and wrist improved the muscle coordination and reduced spasticity. These results indicate the potential for employing EMG signals in closed loop to advance robotic upper limb therapy.

\subsection{Motivation and objective}

Combining a high sensitivity, transparency and high force production required to move the paretic limb all in one machine is often difficult to accomplish. This difficulty increases along with increases in complexity of the robot's geometry. Therefore, for this PhD project, the RehaARM robot was enhanced with myoelectric control in order to increase the system's sensitivity and transparency and be able to detect the individual's intention of movement by measuring residual muscle activity of the paretic upper limb. The enhanced RehaArm (FerRobotics Compliant Robot Technology $\mathrm{GmbH}, \mathrm{AT}$ ) is a compliant robotic arm exoskeleton with 3 DoF for 
shoulder rehabilitation. It mechanically supports the upper arm, lower arm and hand while assisting with shoulder movements. The shoulder joint is actuated while the elbow and wrist are immobilized to attenuate their activity and focus the therapy on the shoulder. Hence, RehaArm with the developed myoelectric interface can be use in early stage shoulder treatment for passive and active single joint shoulder mobilization. The robot implements a soft and slow moving mechanism for a gentle interaction with the subject. This novel system enables performing exercises with the robot in a closed loop, by providing visual feedback on task progress and muscle activity, in order to have a complete sensorimotor integration, wherein the subject is also actively engaged and motivated to initiate and achieve the tasks. To the best of my knowledge, this is the first pneumatically actuated rehabilitation robot for the shoulder, controlled in closed-loop using myoelectric control and visual feedback. This implementation, together with the description of the RehaARM robot, is outlined in sections 2.1, 2.2 and 3.1.

Furthermore, this project assessed whether myoelectric control is more practicable than torque control of the RehaARM robot in a clinical setting (section 3.2). Finally, a phase II clinical trial with the myoelectric controlled RehaARM robot was designed. This trial is registered in ClinicalTrials.gov (identifier: NCT02321254), section 3.3. The aim of this clinical trial was to assess the clinical effectiveness of the roboticassisted upper limb therapy with myoelectric control (in terms of effect size) and to determine the feasibility of incorporating this type of therapy in daily rehabilitation programs. Moreover, the influence of the severity of motor impairment, stroke type and the Stroke to Rehabilitation Interval (SRI) - meaning the distance in time between stroke onset and the start of the rehabilitation treatment - on motor and functional outcomes were also investigated. Finally, I investigated the acceptance of 
this intervention by patients and therapists. The recruiting has been completed and the results are being reviewed by ClinicalTrials.gov for closing the trial.

The objective of this PhD project can be summarized as follows:

Development of a novel, robust and functional myoelectric controlled robotic system for upper limb therapy after stroke, and design and execution of a clinical trial to test the efficacy of the therapy with clinical, kinematic and novel neurophysiological scales based on EMG signals. 


\section{Methodology}

The rehabilitation technologies available for the treatment of upper motor limb impairment after stroke of more severely impaired patients are limited. Therefore, the RehaARM robot and the therapeutic concept of the novel robot-assisted therapy in closed-loop with myoelectric control were implemented and are presented in the following.

Section 2.1 introduces the RehaARM Robot, a robot specifically developed for shoulder rehabilitation [1]. The mechanical structure and operation of the RehaARM robot are explained in detail.

Section 2.2 presents the online closed-loop command interface with EMGthresholding which was designed following SMI principles. In this section, the mechanical adjustments, the software calibration of the system for each individual setting and the closed-control loop with myoelectric and torque control are presented.

Section 2.3 presents the clinical, kinematic and neurophysiological assessments used in the experimental studies (section 3).

\subsection{RehaARM robot}

The mechanical structure of the exoskeleton robot (Figure 1) resembles the human arm anatomy, and the robot's links correspond to human joints. The structure can be adjusted for each subject anthropometry. The humeral and forearm length can be set using mechanical sliders while the adjustment of the shoulder is electrical and mechanical. Electrically, the height of the chair can be changed along the sagittal plane in order to secure the individual shoulder girdle elevation. The transverse chair 
displacement can be modified in order to secure the individual protraction/retraction position of the scapulothoracic joint. Mechanically, the lateral alignment of the scapula can be set by displacing the chair along the coronal plane (Figure 1(d)). In this way, the shoulder joint is centered to the robot axes of rotation, which should cross in the center of the shoulder joint. This can be verified using laser pointers embedded in the robot structure (Figure 1(a-c)).

The elbow is positioned at $90^{\circ}$ of flexion and the wrist in neutral position (i.e., $0^{\circ}$ flexion/extension and $0^{\circ}$ pronation/supination). The lengths of the links L1, L2 and L3 are $67 \mathrm{~cm}, 53 \mathrm{~cm}$ and between 10 to $20 \mathrm{~cm}$ (adjustable), respectively (Figure 1(a, d)). The system provides single arm therapy, but it can be reconfigured easily for the

(a)
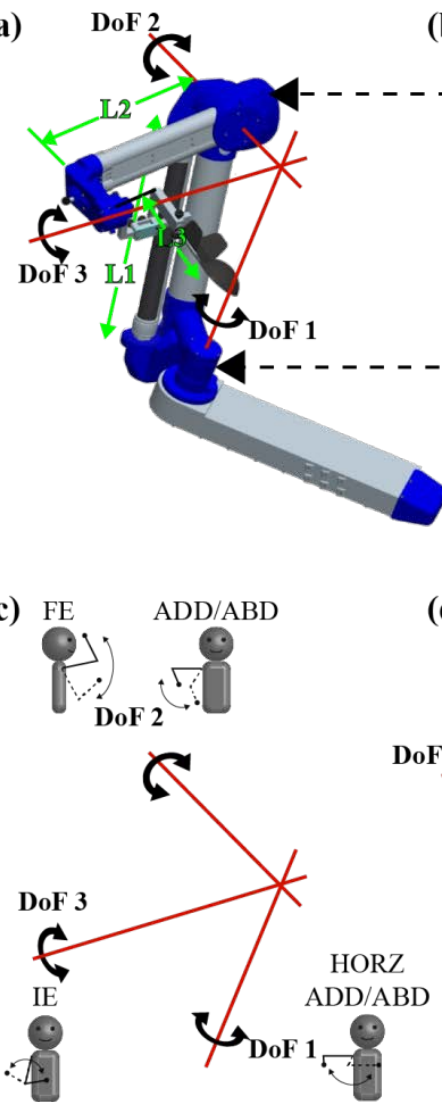

(b)

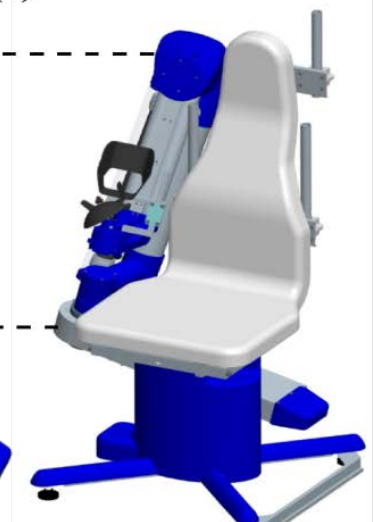

(d)

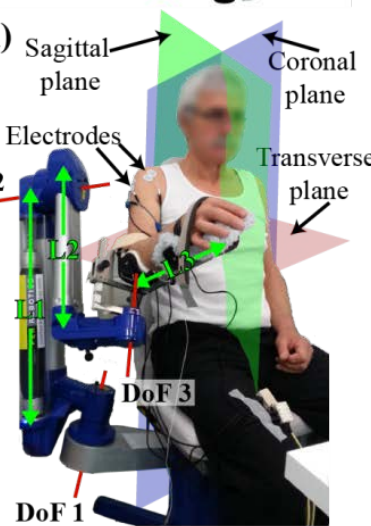

Figure 1: Model of the RehaArm robot. a) Right arm exoskeleton and its three rotational axes for DoF1, DoF2 and DoF3, corresponding to horizontal adduction/abduction (HORZ ADDIABD), adduction/abduction (ADDIABD) and flexion/extension (FE), and internal/external rotation (IE), respectively (c). The robot arm is connected to a chair (b) on which the subject sits comfortably, entirely supporting the arm on the exoskeleton while executing the movements (d). The surface EMG electrodes are mounted on the shoulder muscles to implement EMGdriven control. 
left or right arm to treat patients with brain lesions in the right and left hemispheres. In addition to subject anthropometry, the robot can be calibrated to the arm dynamics. To this aim, the robot measures the weight of the arm at a predefined position (shoulder horizontally abducted and abducted according to the maximum of the subject's passive ROM). The arm model is then used to estimate the gravitational joint moments in an arbitrary position and, based on that, the control signals for the actuators are computed in order to implement feedforward gravity compensation.

RehaArm is able to replicate the principal movements of the shoulder with a proper configuration of its 3 DoF. The first DoF implements a horizontal adduction/abduction (HORZ ADD/ABD), i.e., a movement along the transverse plane. The second DoF implements an adduction/abduction (ADD/ABD) when the exoskeleton arm is horizontally abducted, i.e., a movement along the coronal plane, and a flexion/extension (FE) when the arm exoskeleton is adducted more than $40^{\circ}$, i.e., a movement along the sagittal plane. The third DoF (IE) reproduces the internal/external rotation (IE) (Figure 1(c, d)).

The maximum movement speed of the robot is $5 \%$ and the maximum ranges of motion (ROM) for HORZ ADD/ABD (DoF1), ADD/ABD and FE (DoF2), and IE (DoF3) are $106^{\circ}, 146^{\circ}$ and $160^{\circ}$, respectively. To accommodate the limited motion of the patient's shoulder, the therapist can customize the active ROM of each DoF by setting the minimum and maximum angle positions via software (see section 2.2). The DoF are driven by using antagonistic pairs of pneumatic muscle actuators (Fluid Muscle DMSP, Festo) [39], i.e., six artificial muscles in total. Due to the compressibility of air, the artificial muscles are inherently compliant and they can therefore absorb or at least reduce the contact forces ensuring safety while 
interacting with the patient. If the subject moves the robot from the current reference position, the robot generates restorative force. This force comprises a constant instantaneous reaction, which is proportional to the deviation, and a gradually increasing component (ramp up). The latter can increase only until the total force reaches the maximum value, which is a settable parameter.

\subsection{Online Closed-Loop Command Interface with EMG-Thresholding}

A user-friendly control interface was developed for the RehaArm for the execution of closed-loop, patient-driven experimental protocols. The system includes a multichannel EMG amplifier (EMGUSB2, OTbioelettronica, Torino, IT), a control PC and a standard 22" computer monitor (Figure 2(a)). The robot and the control PC communicated via three unidirectional TCP/IP connections (Figure 2(b)) to set the robot configuration parameters (e.g. maximum torques, maximum ROM), send the desired trajectory (e.g. DoF angles) and receive sensor data for each DoF (e.g. DoF angles and torques) at a sampling frequency of $100 \mathrm{~Hz}$.

The visual feedback modalities for the different experimental protocols were displayed on the monitor and are explained in section 2.2.5 (Figure 2(c1-2)). The robot was controlled in position mode, i.e., the control PC sent a desired position (DoF angles) to the robot, which then exerted forces to drive the subject limb towards that position. The closed-loop control framework also implemented the teaching mode, in which the robot produced zero torques allowing the experimenter to move it freely through the workspace (while the subject was seated in the robot). The trajectory was recorded and could be replayed or analyzed in order to set the range of motion for each DoF, as explained in section 3.1.2.2. 


\subsubsection{Subject Positioning}

The subject was seated comfortably on the chair (Figure 1(b)) and his/her upper limb was strapped to the robotic arm with a soft harness just below the elbow and around the wrist (Figure 1(d)). The link lengths were adjusted according to the subject anthropometry. The experimenter (physiotherapist) took special care to position the humerus correctly, maintaining the parallel alignment of both shoulders before movement. Optimal positioning was necessary in order to activate the muscles responsible for the stability of the shoulder (stability before mobility [40]). Afterwards,

(a) Developed framework in closed loop

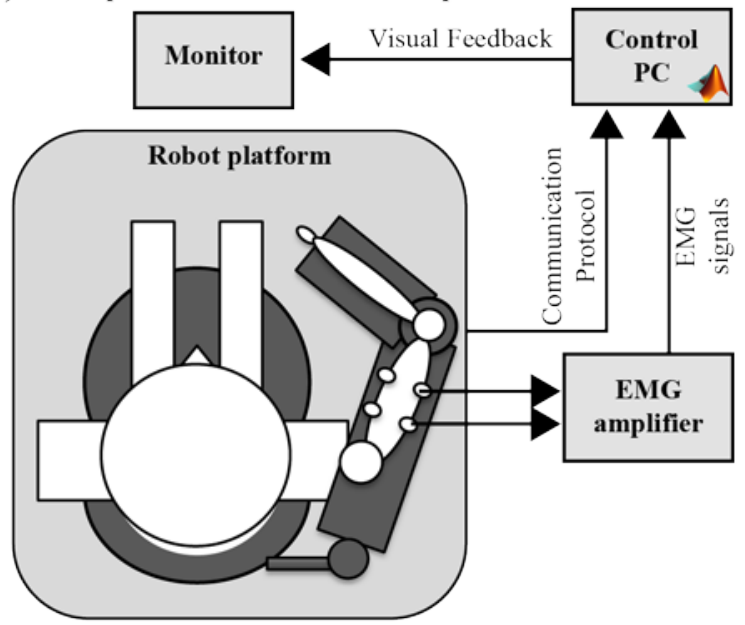

(b) Communication protocol

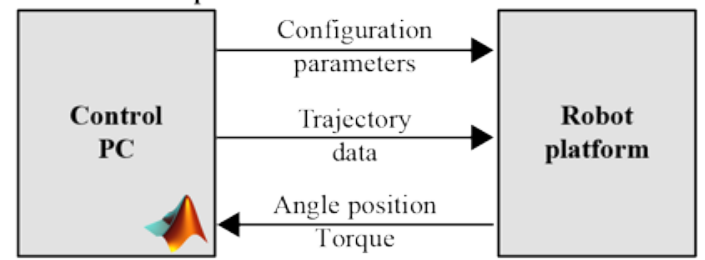

(c) Visual display

1. Single-DoF task

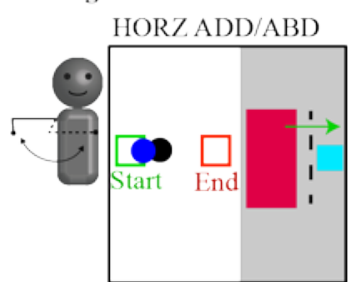

2. EIGHT task: DoF $1+$ DoF 2 HORZ ADD/ABD + FF

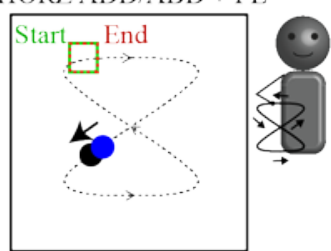

Figure 2: Closed-loop control framework developed for RehaArm. (a) A control PC was connected to an EMG amplifier, the robot and a computer monitor. (b) Three unidirectional TCPIIP connections were used to configure the robot, send the desired position, and receive the sensor data. (c) Visual feedback to the subject displayed on the monitor placed in front of the subject for the single-DoF task (HORZ ADDIABD) (c1) and for the EIGHT task, a simultaneous combination of two single DoF tasks: HORZ ADDIABD and FE (c2). The grey shaded area in c1 was displayed in the tasks with EMG-driven control (section 2.2.5). See text for explanation. 
during the experiment, the physiotherapist continuously controlled the subject's arm and trunk movements in order to avoid compensatory mechanisms.

\subsubsection{EMG Recording and Processing}

For the tasks execution, the surface EMG data was recorded from six shoulder muscles (the triggering muscles). We selected the prime movers of the shoulder joint (pectoralis major, anterior, middle and posterior deltoids) and the rotator cuff muscles (infraspinatus and teres major). The rotator cuff muscles were included since they are the key muscles involved in the glenohumeral stability, dynamic stability and controlled mobility of the shoulder complex. It is known that the lack of glenohumeral stability causes shoulder subluxation, the lack of dynamic stability leads to a non-functional shoulder posture and the lack of controlled mobility impedes smooth movements [40], [41].

For the neurophysiological assessments, the surface EMG data was recorded from sixteen shoulder muscles, including the triggering muscles. The recorded muscles included triceps brachii, lateral (channel 1) and medial heads (channel 2); biceps brachii, short (channel 3) and long heads (channel 4); deltoid, anterior (channel 5), medial (channel 6), and posterior parts (channel 7); superior trapezius (channel 8); rhomboid major (channel 9); brachioradialis (channel 10); supinator (channel 11); brachialis (channel 12); pronator teres (channel 13); pectoralis major, calvicular head (channel 14); infraspinatus (channel 15); and teres major (channel 16). We recorded 16 EMG channels in order to assess the muscle activation of the upper arm [42].

The bipolar electrodes were placed over these muscles following the guidelines of the Surface Electromyography for the Non-Invasive Assessment of Muscles European Community project (SENIAM, [43]). The acquired EMG signals were amplified with a gain of 1000 , band-pass filtered $\left(8^{\text {th }}\right.$ order Bessel filter, bandwidth 
10-500 Hz), sampled at $2048 \mathrm{~Hz}$ and A/D converted with a resolution of 12 bits using a multichannel EMG amplifier connected via USB to the control PC. A reference electrode was placed around the wrist of the right arm. The acquired digitized EMG signals were high-pass filtered ( $5^{\text {th }}$ order Butterworth filter, cutoff 20 $\mathrm{Hz}$ ) to remove motion artifacts. A band-stop filter $\left(5^{\text {th }}\right.$ order Butterworth filter) was applied to remove the power line $50 \mathrm{~Hz}$ noise. The EMG signals were rectified and low-pass filtered ( $5^{\text {th }}$ order Butterworth filter, cutoff $6 \mathrm{~Hz}$ ) to compute a linear envelope for each channel for the myoelectric control. For the neurophysiological assessments, the unrectified EMG-signals were further processed offline in order to obtain the neurophysiological measures (see section 2.3.3).

In the following, recording refers to the acquisition of EMG signals for online control and offline analysis. For study 1 , the surface EMG data from the triggering muscles of stroke patients $(n=12)$ were recorded during each daily session (four sessions in total for study 1). Surface EMG data was also recorded from sixteen shoulder muscles (including the triggering muscles) of healthy volunteers during the first only session ( $n=10)$ (see section 3.1). For study 2, the surface EMG data from the triggering muscles of stroke patients $(n=23)$ were recorded during each daily session (four sessions in total for study 2) and from sixteen shoulder muscles (including the triggering muscles) in session 4 for a subgroup of stroke patients $(n=20)$ (see section 3.2). For study 3, the surface EMG data was recorded from the triggering muscles of stroke patients $(n=20)$ during sessions 2 to 16 and from sixteen shoulder muscles in the session 1 and 17 (seventeenth sessions in total for study 3, see section 3.3).

\subsubsection{Myoelectric Control with Thresholding}

The EMG-driven control was a threshold-based algorithm. To trigger the assistance of the robot, the subject had to activate the selected muscle (task dependent) to 


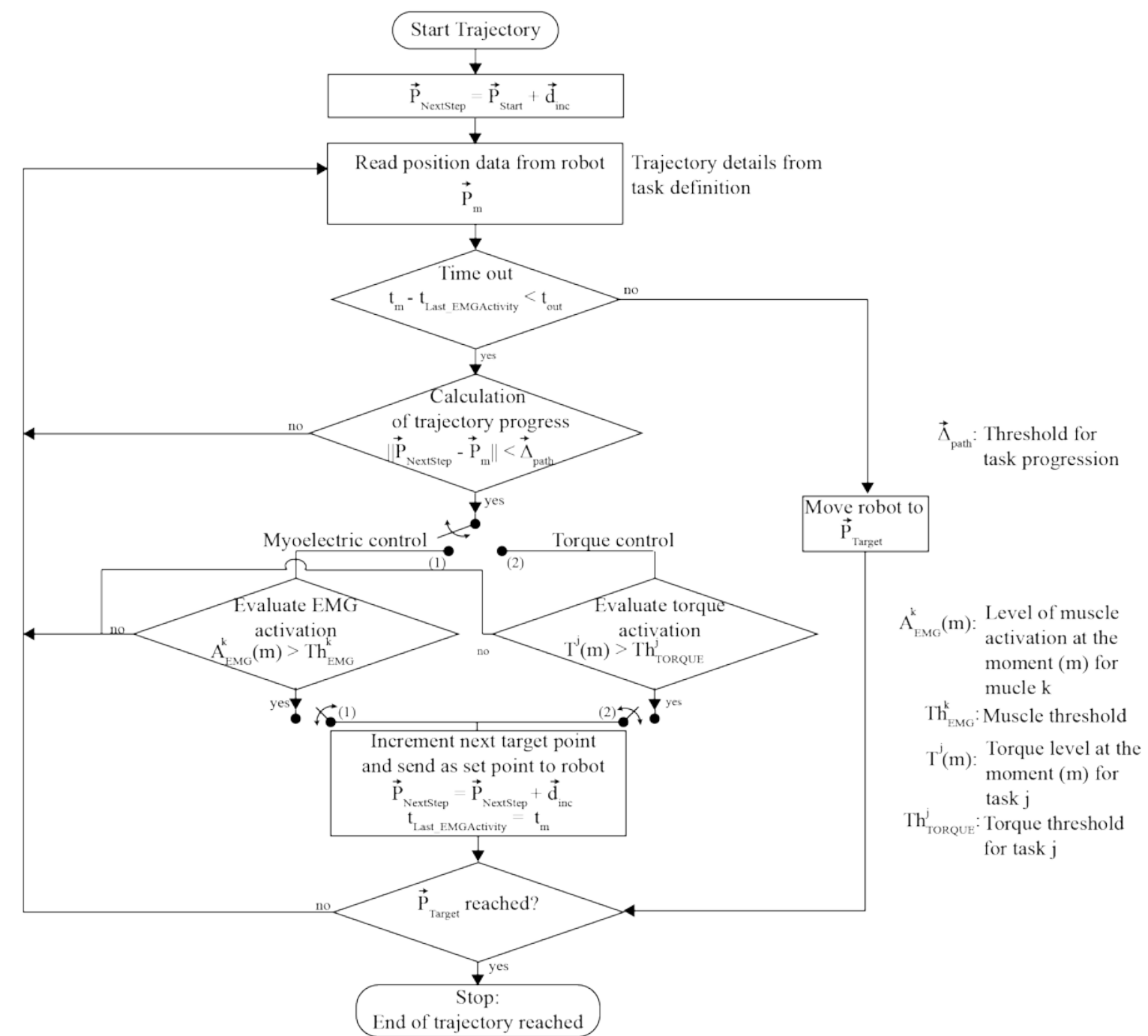

Figure 3: Diagram of the EMG-driven (both switches in position (1)) and torque control (both switches in position (2)) algorithms for the robot's active assistance in the engineering studies 1 and 2 (see sections 3.1.2.2 and 3.2) and the clinical trial (study 3, section 3.3). In myoelectric control, the robot assisted the patient to progressively complete the task if the momentary level of muscle activation $\left(A_{E M G}^{k}(m)\right)$ of the respective muscle at the moment $t_{m}$ for the chosen task exceeded the required muscle threshold $\left(\operatorname{Th}_{E M G}^{k}(m)\right)$ and the patient followed the desired trajectory. In torque control, the robot assisted the patient to progressively complete the task if the momentary torque level $\left(T^{j}(m)\right)$ at the moment $t_{m}$ for the chosen task (j) exceeded the required task threshold $\left(\operatorname{Th}_{\text {TOROUE }}^{j}\right)$ and the patient followed the desired trajectory.

produce above-threshold EMG activity. Visual feedback about the robot position

(shoulder angle) and the level of EMG activity of the triggering muscle was shown to the subject (see section 2.2.5). The robot assisted the patient to complete the task only if the EMG activity of the respective muscle exceeded the required threshold. For online myoelectric control, we obtained the momentary level of muscle activation $\left(A_{E M G}^{k}(m)\right)$ which was the mean of the linear envelope for each channel computed 
over a data window of 100 ms with $50 \%$ overlap, i.e., every $50 \mathrm{~ms}, k=$ $1,2, \ldots, 6$ denotes the muscle and $m$ is the sample number. The full control loop operated therefore at $20 \mathrm{~Hz}$. The calculated momentary muscle activation level was compared to the threshold, as explained next.

The threshold value (eq. 1) for the triggering muscle $\left(T h_{E M G}^{k}\right)$ was set considering the muscle activation during the maximum voluntary contraction $\left(M V C_{E M G}^{k}\right)$ and the EMG channel baseline $\left(B L_{E M G}^{k}\right)$. The threshold values for the triggering muscles corresponded to the 35\% MVC plus 75\% baseline level. The baseline corresponded to the maximum EMG activity at rest:

$T h_{E M G}^{k}=B L_{E M G}^{k}+0.35 \cdot\left(M V C_{E M G}^{k}-B L_{E M G}^{k}\right)$

This assured that the channel threshold was well above the EMG baseline and, at the same time, not too difficult to reach in order to avoid strong muscle fatigue. The calculated threshold could be also manually readjusted online by the therapist to fine tune the control for a specific subject.

The training protocol comprised a set of single-DoF exercises, in which the task for the subject was to move the robot from the initial to the target position along the selected DoF (see section 3.1.2.2). The full control loop is depicted in Figure 3 (with switches in position (1)).

At the beginning of each trial ( $n$ repetitions of a single-DoF task), the robot placed the subject's arm in the initial position $\left(\vec{P}_{\text {Init }}\right)$. If the activation of the triggering muscle was above threshold $\left(A_{E M G}^{k}(m)>T h_{E M G}^{k}\right)$, the host PC incremented the desired position of the robot $\left(\vec{P}_{\text {Nextstep }}=\vec{P}_{\text {Nextstep }}+\vec{d}_{\text {inc }}\right)$ towards the end of the trajectory $\left(\vec{P}_{\text {Final }}\right)$. The robot started moving, providing assistive forces to the subject, and the distance to the desired position was monitored. If the distance was outside of the 
predefined limits, i.e., $\left\|\vec{P}_{\text {Nextstep }}-\vec{P}_{m}\right\|<\vec{\Delta}_{\text {path }}$, where $\vec{P}_{m}$ is the measured position (sensor data) and $\vec{\Delta}_{\text {path }}$ is the tolerance, the robot was lagging and further increment of the desired position was temporary disabled.

The free movement was bounded through the use of virtual walls $\left(\vec{\Delta}_{\text {path }}\right)$ in order to not allow users to exceed certain limits. This tolerance was chosen by the physiotherapist. When the subject was unable to activate the muscle to exceed the threshold before the time-out period (subject too weak), the EMG-driven control loop was terminated, and then the robot fully took over the control to move the limb towards the end of the trajectory $\left(\vec{P}_{F i n a l}\right)$. The therapist were also able to manually activate this option ("move to the end") in order to avoid excessive patient's fatigue. Importantly, this myoelectric control interface can be transferred to other activeassistive robots for upper limb therapy.

\subsubsection{Torque Control}

The torque control was a threshold-based algorithm (switches in position (2) in Figure 3). To trigger the assistance of the robot, the subject had to exert enough force, so that the moment of force (task dependent) was above threshold. Visual feedback about the robot position (shoulder angle) and torque level was shown to the subject (see section 2.2.5). For online torque control, the momentary torque level $T^{j}(m)$ for task $j=1 \ldots 4$ was read from the torque sensors through the communication protocol (Figure 2(b)). The full control loop operated at $20 \mathrm{~Hz}$. The momentary muscle activation level was compared to the threshold which was chosen by the physiotherapist. The torque threshold was also manually adjustable online by the therapist to fine tune the control for a specific subject. 
The training protocol comprised a set of single-DoF exercises, in which the task for the subject was to move the robot from the initial to the target position along the selected DoF (see section 3.2.2).

At the beginning of each trial ( $n$ repetitions of a single-DoF task), the robot placed the subject's arm in the initial position $\left(\vec{P}_{\text {Init }}\right)$. If the momentary torque level was above threshold $\left(T^{j}(m)>T h_{T O R Q U E}^{j}\right)$, the host PC incremented the desired position of the robot $\left(\vec{P}_{\text {NextStep }}=\vec{P}_{\text {NextStep }}+\vec{d}_{\text {inc }}\right)$ towards the end of the trajectory $\left(\vec{P}_{\text {Final }}\right)$. The rest of the algorithm worked the same as for myoelectric control (see section 2.2.3). The torque control loop was terminated and the robot fully took over the control to move the limb towards the end of the trajectory $\left(\vec{P}_{\text {Final }}\right)$ when the subject was unable to exert enough residual torque to exceed the threshold before the timeout period (subject too weak). The therapist was able as well to manually activate this option as for myoelectric control.

The minimum working value for the torque level that could be set for DoF 1, DoF 2 and DoF 3 was $1 \mathrm{Nm}, 0.5 \mathrm{Nm}$ and $1 \mathrm{Nm}$, respectively, in order to avoid oscillatory motions of the robot. Oscillatory motions are a drawback of pneumatic robots and are due to a dynamic delay of the pressure response. The capability of the RehaARM robot to measure these torque values from $0.5 \mathrm{Nm}$ to $1 \mathrm{Nm}$ together with the compliant actuation of the RehaARM is not bad, it corresponds to the moment of force of small masses, 50 to $100 \mathrm{gr}$, respectively, rotating around an axis at a distance of $1 \mathrm{~m}$. But it is limited, especially when the robot is supposed to assist more severely impaired patients. These users will always feel some residual apparent inertia and not free at motion. 


\subsubsection{Visual Feedback}

To assist and motivate the subjects when performing the task, they were provided with visual feedback (Figure 2(c)), depicting initial $\left(\vec{P}_{\text {Init }}\right.$, green square), final $\left(\vec{P}_{\text {Final }}\right.$, red square) and current position ( $\vec{P}_{m}$, blue ball). In the single-DOF target-tracking tasks (section 3.1.2.1), the desired position (target) was displayed (black ball) while the grey shaded area was not shown. If the robot was driven using myoelectric control (sections 3.1.2.2, 3.1.3.2 and 3.3), the black ball was not shown and the feedback (grey shaded area in Figure 2(c1)) included the momentary muscle activation level $\left(A_{E M G}^{k}(m)\right.$, light blue square) together with the threshold level $\left(T h_{E M G}^{k}\right.$, black dashed line) were shown. If the robot was driven using torque control (section 3.1.3.2), the black ball was not shown and the feedback (grey shaded area in Figure 2(c1)) included the momentary torque level $\left(T^{j}(m)\right.$, light blue square) together with the threshold level $\left(T h_{T O R Q U E}^{j}\right.$, black dashed line) were shown. In addition, the area of wrong activation was indicated as a red box. The upper border of this area corresponded to zero activation of the triggered muscle. If the muscle activation (light blue square) was within the area of the wrong activation, this indicated that the subject contracted wrong muscles (i.e., antagonists to the triggered muscle), pulling in the wrong direction. For torque control the upper border of this area corresponded to zero torque and if the momentary torque level (light blue square) was in this area, the subject was moving the arm in the opposite direction.

The user's central nervous system was incorporated into the control loop through a visual feedback in combination with kinesthetic, proprioceptive and tactile feedback. This allows the user to accomplish position control, with the RehaARM robot acting as a forward-loop strength amplifier. 


\subsection{Assessments}

Clinical, kinematic and neurophysiological assessments were used in this project in order to determine the feasibility and efficacy of the RehaARM robot and its therapeutic concept.

\subsubsection{Clinical assessment}

The clinical assessments included impairment outcome measures and disability measures.

\subsubsection{Impairment outcome measures}

These outcomes assess impairments in body function, structure or system (including psychological). In this study, I just included body function impairments.

- The Fugl-Meyer Assessment of Motor Recovery after Stroke (FMA) is a disease-specific impairment index designed to assess motor function, balance, sensation qualities and joint function in hemiplegic post-stroke patients ([44], [45]). The score goes from 0 to 152. Minimum detectable change (MDC) and minimum clinically important difference (MCID) are not established ${ }^{6}$.

- The upper extremity (UE) part of the Fugl-Meyer Assessment of Motor Recovery after Stroke (FMA-UE). This motor impairment test consists of 33 items that assess voluntary movement, reflex activity, grasp, and coordination on a scale, with total scores ranging from 0 (no function) to 66 points (normal function). A MDC of 5.2 has been determined based on a study with a study with 14 subjects who suffered a single, unilateral stroke within 6 to 26 months before the admission into the study [46].The threshold for the MCID in patients with minimum to moderate chronic impairment after stroke is about 5

\footnotetext{
${ }^{6}$ http://www.rehabmeasures.org
} 
points (range 4.25-7.25) depending on the movement domain of the test) [47].

- Modified Ashworth Scale (MAS) assesses the amount of resistance or tone (spasticity) perceived by the examiner subjectively as the limb is moved through its full ROM. The tone can be no resistant or rigid [48], [49]. The scale goes for from 0 (no resistance) - 20 (rigidity). MDC and MCID are not established ${ }^{6}$.

- Reaching Performance Scale (RPS) assesses the ability of the subjects and the quality of the movement in reaching an object (a cone). The cone is placed either close to the subject $(4 \mathrm{~cm})$ or farther away $(30 \mathrm{~cm})$. The subject is asked to reach the cone, pinching it on its point. The observer evaluates only the reaching movement and not the strength of the pinch. The score ranges from 0 to 36 . Both tasks (close and far target) assesses the same six components using a 4-point ordinary scale; from 0 to 3 . The six components are: trunk displacement, movement smoothness, shoulder movement, elbow movement, quality of grip and overall evaluation [50]. MDC and MCID are not established ${ }^{6}$.

\subsubsection{Activity scales}

These measures focused primarily on the identification of limitations in activity.

- Functional Independence Measure (FIM) consists of 18 items assessing 6 areas of function (self-care, sphincter control, mobility, locomotion, communication and social cognition). These fall into 2 basic domains; motor (13 items) and cognition (5 items) [51]. The score ranges from 18 (complete dependence/total assistance) to 126 (complete independence).The MCID has been identified for the stroke population based upon ratings of clinical change 
made by physicians shortly following discharge from stroke rehabilitation [52]. The MCID is 22, 17 and 3 for the total FIM, motor FIM and cognitive FIM, respectively. We used here the motor FIM separately in order to assess the physical improvement.

- Nine-hole Peg Test (NHPT) is a timed measure of fine finger dexterity [53], [54]. The subject is asked to insert nine pins into the pegboard as quickly as possible. The assessment in this study was done as the number of pins inserted by the subject in $50 \mathrm{~s}$ or by the time needed to insert the nine pins. We just used the number of inserted pins as NHPT variable. The time was not considered in this study. MDC and MCID are not established ${ }^{6}$.

\subsubsection{Kinematic assessments}

The active ROM of motion for the movements shoulder abduction (shoulder_ABD), shoulder flexion (shoulder_FLEX) and elbow flexion (elbow_FLEX) were assessed using the Pablo robot from Tyromotion. Pablo is a rehabilitation robot for arm and hand therapy. The Pablo system has a sensor handle equipped with position sensors to measure ROM of wrist, elbow and shoulder. The patient simply grasps the handle and the hand is fixed to the handle with a strap. The kinematic measures of the paretic limb took approx. $3 \mathrm{~min}$. Subjects were instructed to perform the shoulder_ABD and the shoulder_FLEX with the elbow in $180^{\circ}$ extension if possible depending on residual motor control. The therapist stopped the measurements if the patient compensated in order to avoid gains due to compensation. Patient started all movements with the hand in neutral position $\left(0^{\circ}\right.$ pronation/supination).

\subsubsection{Neurophysiological assessments}

Voluntary movements are produced by the functional integration of several motor cortical areas, such as the primary and supplementary motor cortical areas, and 
spinal circuitries. The synergistic activation of a set of muscles involved in a movement is a transformation of a low dimensional supraspinal signal set (known as motor primitives [42]) from the central nervous system (CNS) into a high dimensional signal set to the muscles. This transformation taking place in the spinal cord has been modeled in [55], [56], and is described in the so-called synergy theory. The synergy concept has two parts, the forward and the inverse model. The forward model (Figure 4(a)) describes the muscle patterns (time-invariant muscle synergies) generated by supraspinal motor commands (time-dependent coefficients or primitives) which can be obtained by measuring the surface EMG signals. The inverse model is the counterpart of the forward model and is used to obtain the supraspinal motor commands given the muscle synergies and the surface EMG signals.

In this study, the forward model was used in order to obtain the muscle synergies of 16 muscles for each task which subjects performed with the robot. The muscle synergies were obtained from the surface EMG signals. For this the acquired EMG signals were first high-pass filtered with a window-based finite impulse response filter $\left(50^{\text {th }}\right.$ order, cutoff frequency of $50 \mathrm{~Hz}$ ) to remove motion artifacts, rectified, low-passfiltered $\left(50^{\text {th }}\right.$ order, cutoff frequency of $\left.20 \mathrm{~Hz}\right)$ to remove noise, and then integrated over $25 \mathrm{~ms}$ intervals to obtain the muscle activation envelopes $\left(A S_{E M G}^{i}\right)$ which are always positive, Figure 4 . The muscle synergies $(S)$ and the corresponding activation coefficients $(P)$ were extracted from the muscle activations envelopes $A S_{E M G}^{i}$ using the NMF algorithm [57]. The Non-Matrix Factorization (NMF) models the muscle activations as a linear combination (eq. 1) of several muscle synergies, each activated by a time-dependent coefficient. The muscle activations are always positive since a muscle can only contract actively, but not expand. Therefore, the 
(a)
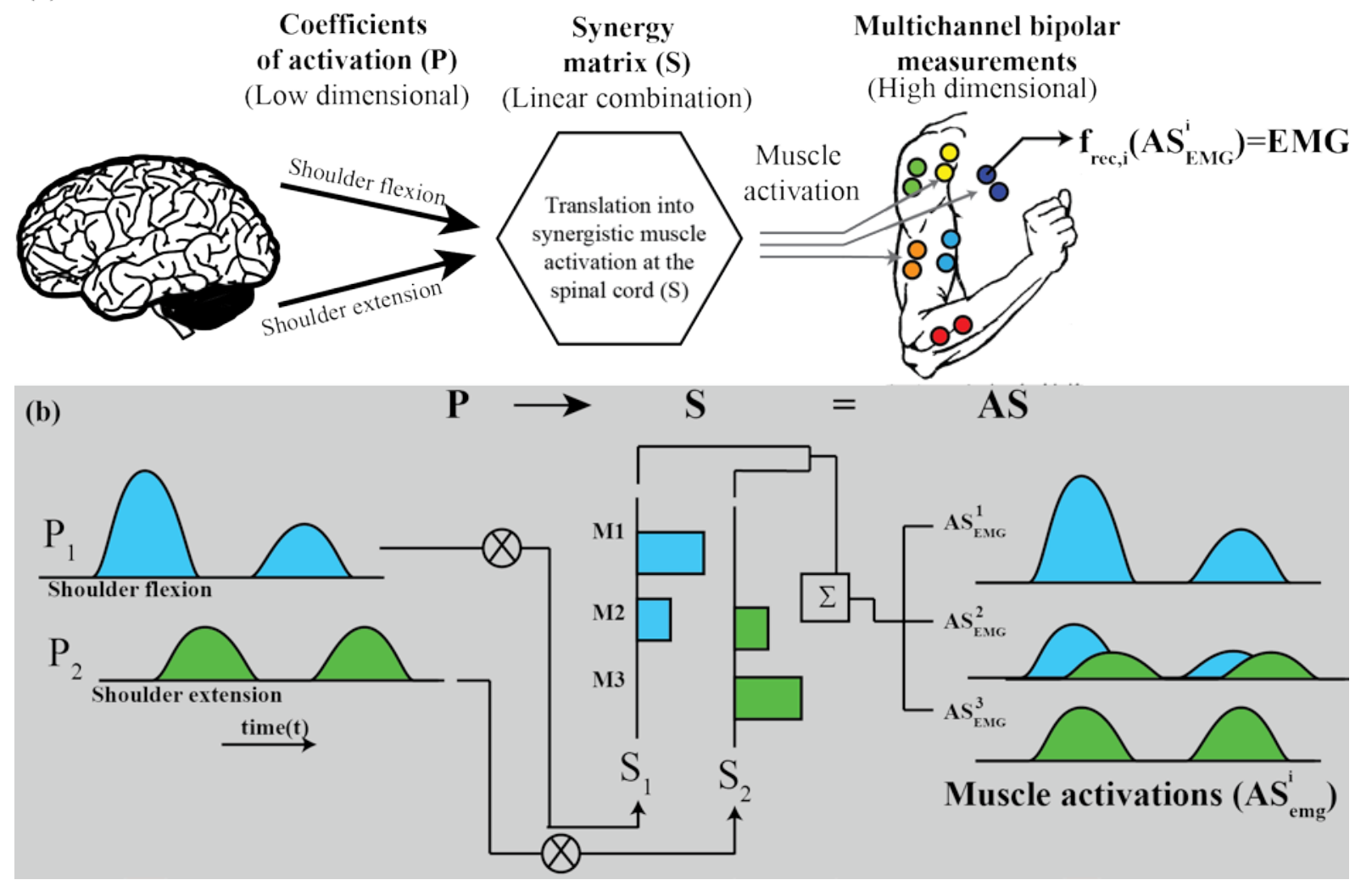

Figure 4: Forward synergy concept for the control of the shoulder flexion/extension. In (a) from the motor cortex, the low dimensional motor commands (primitives or activation coefficients, P) are sent to the spinal cord (S). In the spinal cord, $P$ is transformed into the high dimensional muscle activation signals $A S_{E M G}^{i} i=1,2,3$, necessary to execute the intended movement. Finally, the muscle activation is recorded by the multi-channel bipolar surface EMG system. In (b), the model is schematically illustrated. It can be visualized how the recorded EMG signals can be reconstructed by linearly combining several time-invariant muscle synergies $\left(S_{1}, S_{2}\right)$, each activated by a distinct time-dependent coefficient waveform (primitives, $P_{1}, P_{2}$ ). Each of the two synergies $(S$, dimension $3 \times 2)$ weights the coefficients ( $P$, dimension $2 \times$ nSamples) in order to obtain three muscle activations ( $A S$, dimension $3 \times$ nSamples). 'nSamples' is the number of samples per task (nSamples $=$ time $^{\star} 2048 \mathrm{~Hz}$ ), the number of synergies is two, the number of muscles is three and there are two primitives $\left(P_{1}\right.$ for shoulder flexion and $P_{2}$ for shoulder extension).

obtained primitives and synergies are also positive. For example, $A S_{E M G}^{2}$ in (Figure 4(b)) is a combination of the coefficients $P_{1}$ and $P_{2}$ weighted by muscle $\left(M_{2}\right)$ from synergies $S_{1}$ and $S_{2}$. In this example, nMuscles equals 2, nSamples is the number of samples per task and nSynergies equals 2.

$A S=P X S$

, $\boldsymbol{A S}$ is the matrix of muscle activation and has a dimension of [nMuscles X nSamples], $\boldsymbol{P}$ is the matrix of activation coefficients (primitives) and has dimension of [nMuscles $\mathrm{X} n$ Synergies] and $S$ is the synergy matix and has a dimension of [nSynergies $\mathrm{X}$ nSamples]. 
In this way, the muscle synergies per task for each subject group (healthy subjects and patients) in each control modality, torque and myoelectric control, were calculated. The primitives were not used in this study, but they were inspected in order to confirm correct synergy extraction. As expected, the two primitives per task were two waveforms with alternating activity similar to the primitives $\left(P_{1}, P_{2}\right)$ in Figure 4(b). The number of muscle synergies for the NMF algorithm was set a priori to 2 because we have single-DoF movements in two directions (e.g. Shoulder flexion and extension).

NMF presents ambiguities of scaling and permutation like other factorization algorithms. The scaling ambiguity means that it is possible to identify only the relative, and not the absolute, activations between synergistic muscles. In this study, the scaling ambiguity was adjusted for by normalizing each synergy with respect to the level of activity of the most active muscle in that synergy. After this normalization, the maximum value in each column of the synergy matrix was equal to 1 . The ambiguity of permutation is not relevant in the synergy context because there are just two synergies.

The muscle synergies were then used to obtain the neurophysiological assessments EMG-S and TORQUE-S. The median of the muscle synergies of all healthy subjects $(n=10)$ was used as the reference muscle activation for using the robot with myoelectric (EMG-S) or torque control (TORQUE-S). EMG-S is the overall similarity between sets of muscle synergies from the healthy subjects (reference muscle activation) and a patient when using myoelectric control. First, the similarity for each task with myoelectric control was calculated as the similarity (cosine similarity in eq. 3) between the two synergies of the healthy subjects group (reference muscle activation for the corresponding task, $\boldsymbol{A}$ in eq. 3) and the two synergies of a patient 
for the corresponding task ( $\boldsymbol{B}$ in eq. 3). The overall similarity for a patient was then the median of the similarities of all tasks.

TORQUE-S is the overall similarity between sets of muscle synergies from the healthy subjects group (reference muscle activation) and a patient when using torque control. The overall similarity was calculated as for EMG-S. similarity $=$ $\cos (\theta)=\frac{\boldsymbol{A} \cdot \boldsymbol{B}}{\|\boldsymbol{A}\|\|\boldsymbol{B}\|}$

In other words, the similarity is a measurement that compares the relative contribution of the muscles involved in a movement. Therefore, EMG-S and TORQUE-S are measurements that compare the relative contribution of the muscles involved in a movement between healthy subjects (reference muscle activation) and a patient when patients drove the robot with myoelectric and torque control, respectively. 


\section{Experimental Studies of the Newly Designed Robotic- Assisted Therapy}

Three studies were performed to evaluate the result of this development. Section 3.1 presents a first investigation on the feasibility of using a closed-loop myoelectric control system with nine healthy subjects and 12 stroke patients in a clinical set up. It also presents the acceptability and safety of the therapy. The results on both healthy subjects and stroke patients evidenced that the proposed RehaArm and the proposed therapeutic concept can be used in the clinical practice with a range of parameters to selectively and gradually modulate the motor response.

Section 3.2 presents a second investigation on the practicability and ease of use of closed-loop myoelectric control versus closed-loop torque control of the RehaARM with 23 stroke patients. The results showed that myoelectric control with simple EMG-thresholding is more practical than torque control for robotic-assisted therapy of upper limb motor impairment.

Finally, section 3.3 presents a third and final investigation on the effectiveness of using a robotic-assisted therapy of the upper limb with closed-loop myoelectric control on a population of 20 stroke patients with severe, moderate and mild impairments as assessed by the Fugl-Meyer Scale of the upper extremity.

\subsection{Study 1: A Novel Pneumatic EMG-driven Robotic System for Shoulder Rehabilitation after Stroke}

(This section is based on a manuscript submitted to BioMed Central for consideration [1]; therefore text, results and graphics taken from this manuscript are not cited explicitly) 


\subsubsection{Abstract}

Objective. This engineering study presents the RehaArm robot, a novel 3DoF active, assistive robot designed for shoulder rehabilitation. The technical characteristics of the robot and the therapeutic modalities in closed loop control are described. Approach. The robotic-assisted therapeutic system was tested using two experimental protocols in order to demonstrate the robot operation in a clinical setup: (1) anti-gravity support during single and simultaneous DoF activation; and (2) antigravity support and EMG-driven control. The experiments were performed with nine healthy subjects and 12 stroke patients. The 12 stroke patients only used EMG-driven control during four daily sessions. During myoelectric control, subjects had to actively and continuously drive the robot to complete the tasks in closed-loop using visual feedback of muscles signals. The robot assisted the patient if the EMG activity surpassed an individually settable EMG threshold value for each movement. The tasks were almost planar, single-DoF tasks for the shoulder. These tasks were functional, but yet simple, for training with more severely impaired patients. For myoelectric control, the subjects' goal was to achieve a task completion rate (CR) of 40 repetitions (5 repetitions $\times 4$ single-DoF tasks $\times 2$ directions) during 20 min. Main Results. Using myoelectric control, the group of healthy subjects was able to execute 40 task repetitions in 20 min. Over all days, the mild impairment group (group B, $n=6)$ performed significantly better $(p<0.0001)$ than the severe-to-moderate impairment group (group $A, n=6$ ). For group $B$, the median $C R$ over all days was 39 task repetitions per daily session within 20 min with Q3 $=40$ and Q1 $=32.5$ against a median CR of 24 repetitions with $\mathrm{Q} 3=29$ and $\mathrm{Q} 1=14$ for group $\mathrm{A}$. Both patient groups performed tendentially better over time. Consecutively from day 1 to day 4 , group A executed a median of 18.5, 20, 27 and 24.5 task repetitions while group B executed a median of $34.5,38.5,38$ and 40 task repetitions in 20 min, but there was 
no significant difference in the CR over time. Significance. Patients including those more severely impaired were able to self-initiate and control the RehaArm robot using EMG-signals for shoulder therapeutic training, improving their movement completion rate over consecutive days. We conclude that the presented system can be flexibly, simply and safely employed in the clinic for shoulder therapy in impairments of upper limb motor function for a larger stroke population with different impairments: severe, mild and moderate. The system can be used with a range of parameters to selectively and gradually modulate the motor response.

\subsubsection{Methods}

The system has been tested in two main conditions: the evaluation of the Robot Compliant Operation (section 3.1.2.1) and the Clinical Evaluation Using EMGControl (section 3.1.2.2). Two trained physiotherapists were involved. One physiotherapist contributed to the experimental design and the other was in charge of executing the experimental protocols. In addition, an engineer (mostly the author of this thesis) was always present during the sessions.

\subsubsection{Robot Compliant Operation}

The test included three healthy male subjects (age $=29 \pm 6$ yrs).

The robot provided antigravity support. It generated forces to hold the limb in a desired position. Meanwhile, the subject was required to exploit the robot compliance

Visual display

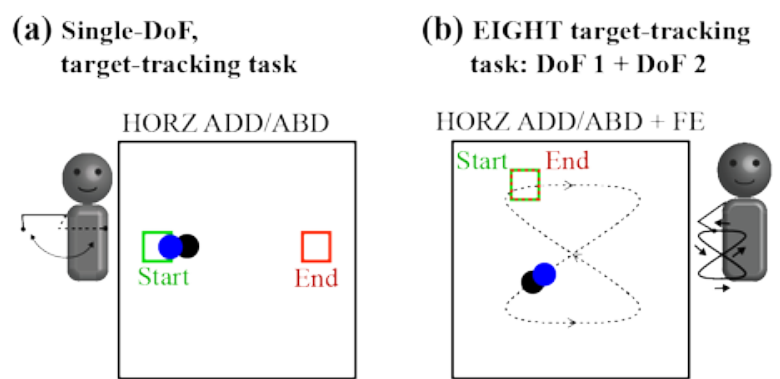

Figure 5: Visual feedback to the subject displayed on the monitor placed in front of the subject for the single-DoF target tracking task (HORZ ADDIABD) (a) and for the EIGHT target-tracking task, a simultaneous combination of two single DoF tasks: HORZ ADDIABD and FE (b). 
and actively move the limb around the current set point, as defined by the experimental task. For these tests, the myoelectric control (section 2.2.3) was not employed, and the robot command was only to stay in the reference position. When the subject deviated from the reference position, his movement was counteracted by the corrective torques generated by the robot. Maximal possible deviation from the reference depended on the subject's effort and the robot maximal torque, where the latter is an adjustable parameter. Essentially, this protocol was a combination of assistive (antigravity support) and resistive (restorative torques) training, where the torque of each training modality could be adjusted from zero to the maximal torque that the robot can generate.

The starting position of the shoulder was horizontal adduction $\left(45^{\circ}\right)$, plus flexion $\left(70^{\circ}\right)$ and $0^{\circ}$ of humeral rotation. In the following, the two tasks comprised in the protocol are explained.

For the single-DoF target-tracking tasks (Figure 5), the subjects had to follow a target (black ball) as it moved along a straight line from the initial to the final position at a constant speed. Two movement trajectories were used, a straight line along HORZ ADD/ABD (Figure 5(a)) or along FE DoF. In the EIGHT target-tracking task (Figure 5(b)), the subjects tracked a trajectory shaped as a number eight positioned parallel to the coronal plane, activating two DoFs simultaneously (i.e., HORZ $A D D / A B D$ and $F E)$. The direction of movement along the trajectory was displayed to the subject.

\begin{tabular}{|c|c|c|c|c|}
\hline \multicolumn{5}{|c|}{ Table 1: conditions for the single-dof tracking task } \\
\hline Condition & $\begin{array}{c}\text { Trajectory } \\
\text { Length }[\mathbf{c m}]\end{array}$ & $\begin{array}{c}\text { Maximum } \\
\text { torque }[\mathbf{N m}]\end{array}$ & $\begin{array}{c}\text { Speed of target } \\
\text { Movement [\%] }\end{array}$ & $\begin{array}{c}\text { Holding } \\
\text { Time [s] }\end{array}$ \\
\hline C1 & 10 & 0 & 10 & 0.01 \\
\hline C2 & 10 & 0 & 30 & 0.01 \\
\hline C3 & 10 & 10 & 10 & 0.01 \\
\hline C4 & 10 & 10 & 30 & 0.01 \\
\hline C5 & 10 & 10 & 30 & 5 \\
\hline C6 & 10 & 0 & 10 & 5 \\
\hline
\end{tabular}


Table 2: robot configuration setting

\begin{tabular}{|c|c|c|}
\hline Variable & Effect Description & $\begin{array}{l}\text { Values used } \\
\text { [min, max] }\end{array}$ \\
\hline $\begin{array}{l}\text { Maximum } \\
\text { torque }\end{array}$ & $\begin{array}{l}\text { Set only for the DoF involved in the task; it limits the } \\
\text { corrective force that the robot exerts on the subject's arm }\end{array}$ & $\begin{array}{c}{\left[0,(5,10)^{*}\right]} \\
{[\mathrm{Nm}]}\end{array}$ \\
\hline $\begin{array}{l}\text { Trajectory } \\
\text { length }\end{array}$ & $\begin{array}{l}\text { Larger deviation from the reference position results in the } \\
\text { stronger corrective robot torque }\end{array}$ & {$[10,20][\mathrm{cm}]$} \\
\hline $\begin{array}{c}\text { Speed of } \\
\text { target } \\
\text { movement }\end{array}$ & $\begin{array}{l}\text { Forces due to the viscosity (i.e. higher motion speed } \\
\text { results in stronger resistive torque) }\end{array}$ & {$[10,30][\% / s]$} \\
\hline $\begin{array}{c}\text { Holding } \\
\text { time }\end{array}$ & $\begin{array}{l}\text { Time to hold the robot at the final position for the buildup } \\
\text { of the resistive robot torque }\end{array}$ & {$[0.01,5][\mathrm{s}]$} \\
\hline
\end{tabular}

In the single-DoF target-tracking tasks, we tested six conditions characterized by different values of the robot configuration parameters (Table 2 and Table 1). Each subject performed 10 repetitions in each condition. In the EIGHT task, the maximum resistive torque was changed from soft (i.e. minimum torque) to stiff (i.e. maximum torque) in each DoF individually as well as in both DoF concurrently, resulting in four conditions in total (Table 3), while the parameter "holding time" was not applicable for this task. Each subject performed three repetitions in each condition. This protocol was executed in only one daily session of one hour including subject preparation.

\subsubsection{Clinical Evaluation Using EMG-Control}

Six healthy volunteers (two male and four female subjects, $58.5 \pm 14 \mathrm{yrs}$, right dominant) and 12 right hemiparetic stroke patients (Table 4) meeting study inclusion criteria participated in the study. The inclusion criteria for stroke patients were: firstever supratentorial stroke (ischemic and/or hemorrhagic) in the left hemisphere, with the score between 1 and 3 in the upper limb sub-item of the Italian version of the National Institutes of Health Stroke Scale (IT-NIHSS). The IT-NIHSS score was considered as a reliable criterion for assessing the existence of residual voluntary motor contraction, which was a necessary condition to use the myoelectric control. The study exclusion criteria were: Mini Mental State Examination (MMSE) 
Table 3: conditions for the eight tracking task

\begin{tabular}{|c|c|c|c|c|}
\hline Condition & $\begin{array}{c}\text { FE DoF } \\
\text { Maximum torque [Nm] }\end{array}$ & $\begin{array}{c}\text { ABD DoF } \\
\text { Maximum torque [Nm] }\end{array}$ & $\begin{array}{c}\text { Speed of } \\
\text { Movement [\% } / \mathbf{s}]\end{array}$ & $\begin{array}{c}\text { ROM } \\
{\left[{ }^{\circ}\right]}\end{array}$ \\
\hline E1 & 0 & 0 & 5 & 50 \\
\hline E2 & 0 & 5 & 5 & 50 \\
\hline E3 & 5 & 0 & 5 & 50 \\
\hline E4 & 5 & 5 & 5 & 50 \\
\hline
\end{tabular}

score $<20 / 30$, score (tau points) in the Token Test for verbal comprehension $<58 / 78$ and non-stabilized fractures.

The patients were classified in two groups according to the FMA-UE. Patients with an FMA-UE score $<=38 / 66$ were included in the severe-to-moderate group (A) and those with a FMA-UE score $>38 / 66$ were included in the mild stroke group (B) (Table IV). Patients were classified according to their motor impairment in order to homogenize the subject groups and achieve more relevant clinical insights, as the previous studies have demonstrated that the treatment outcome can depend substantially on the level of impairment at baseline [26], [58].

The exercises included movements along a single DoF (Table 5), similarly to the target-tracking tasks described in the previous section 3.1.2.1. However, in this protocol subjects used myoelectric control to move the robot (myoelectric control with simple EMG-thresholding, section 2.2.3), were not required to track a target and were able to move at their own pace. The HORZ ADD/ABD, ADD/ABD, FE and IE single-DoF movements, selected as the clinical tasks, represent basic shoulder and scapula movements. The single-DoF movements 1, 2 and 4 (Table 5) are performed mainly by activating the shoulder muscles pectoralis major and the three deltoids, with the partial involvement of the rotator cuff muscles. For example, during $90^{\circ}$

\begin{tabular}{|c|c|c|c|}
\hline & Frequency & median & Q1-Q3 \\
\hline Age & & 68.5 & $55.1-76.4$ \\
\hline Gender (M / F) & $8 / 4$ & & \\
\hline Months since stroke & & 4.8 & $3.8-7.2$ \\
\hline FMA-UE $(\leq 38 />38)$ & $6 / 6$ & & \\
\hline
\end{tabular}

*Q1 and Q3 are the first and third Quartile. 


\begin{tabular}{|c|c|c|c|c|}
\hline $\begin{array}{c}\text { Robot DoF } \\
\text { (Corresp. single- } \\
\text { DoF task) }\end{array}$ & Task & $\begin{array}{c}\text { Triggering } \\
\text { muscle }\end{array}$ & Task & $\begin{array}{c}\text { Triggering } \\
\text { muscle }\end{array}$ \\
\hline $\mathbf{1}$ & $\begin{array}{c}\text { HORZ } \\
\text { ADD }\end{array}$ & $\begin{array}{c}\text { Pectoralis } \\
\text { major }\end{array}$ & $\begin{array}{c}\text { HORZ } \\
\text { ABD }\end{array}$ & $\begin{array}{c}\text { Posterior } \\
\text { deltoid }\end{array}$ \\
\hline $\mathbf{2}$ & ADD & Teres major & ABD & $\begin{array}{c}\text { Medium } \\
\text { deltoid }\end{array}$ \\
\hline $\mathbf{3}$ & $\begin{array}{c}\text { Int rot } \\
(\mathrm{I})\end{array}$ & Teres major & Ext rot (E) & $\begin{array}{c}\text { Infraspi- } \\
\text { natus }\end{array}$ \\
\hline $\mathbf{4}$ & $\begin{array}{c}\text { Flexion } \\
(\text { FLEX) }\end{array}$ & $\begin{array}{c}\text { Posterior } \\
\text { deltoid }\end{array}$ & $\begin{array}{c}\text { Extension } \\
\text { (EXT) }\end{array}$ & $\begin{array}{c}\text { Anterior } \\
\text { deltoid }\end{array}$ \\
\hline
\end{tabular}

flexion, $60^{\circ}$ are supported by the glenohumeral joint (humeral elevation) and $30^{\circ}$ by the scapular muscles (upward rotation of the scapulothoracic joint), which is known as the 2:1 ratio scapulohumeral rhythm [40]. The IE exercise (Table 5) activates more the muscles controlling the scapula, such as, rotator cuff muscles, teres major and infraspinatus (Figure 1 (d)).

For each exercise, a trained physiotherapist selected the triggering muscles based on the pilot tests. The triggering muscle was always either the most activated one or it belonged to the group of most activated synergistic muscles (Table 5). The individual maximum ROM of the shoulder for each single-DoF task was adjusted by the physiotherapist using the graphical user interface (GUI) during the first session (and if necessary, readjusted in the following sessions). The physiotherapist moved the exoskeleton along the task DoF from minimum to maximum excursion, so that the obtained ROM corresponded to the comfortable ROM for the specific patient.

To obtain the EMG baseline of each channel $\left(B L_{E M G}^{k}\right)$, the robot was positioned in $55^{\circ}$ of horizontal adduction, $35^{\circ}$ of flexion and $0^{\circ}$ of rotation. The subject was asked to relax and the EMG signals were recorded for 1s. The EMG baseline was computed as the maximum value of the rectified envelope of the recorded EMG signal. To measure the MVC of the triggering muscles $\left(M V C_{E M G}^{k}\right)$, the robot was positioned in the middle between the initial and final positions in the task for which that muscle was the trigger muscle (Table 5). Subsequently, the subject was asked to push the exoskeleton as strong as possible towards the final position (task 
relevant direction) maintaining the contraction for $3 \mathrm{~s}$. The $M V C_{E M G}^{k}$ was computed as the maximum value of the rectified envelope of the EMG signal recorded in the last second. The EMG baseline and MVC value were calculated at each trial and session. One trial consisted of 5 repetitions of a single-DoF task; therefore the session compromised four trials, one trial per task.

The parameters for the myoelectric control were set to $\vec{d}_{\text {inc }}=5^{\circ}, t_{\text {out }}=40 \mathrm{~s}$ and $\vec{\Delta}_{\text {path }}$ to $13^{\circ}, 7^{\circ}, 10^{\circ}$ and $7^{\circ}$ for DoF1 to DoF4, respectively. The maximum torque was configured to $20 \mathrm{Nm}$ for HORZ ADD/ABD and IE, and 30Nm for ADD/ABD and FE DoFs. The torque was set to high values, so that the robot supported the arm along the selected task, providing active assistance to the patient while performing the movement, and at the same time limiting the excursions along the other DoF.

Patients performed the protocol in four consecutive days, one session daily. The session lasted for approximately 40 min including a 10-min preparation time. During each session, the four single-DoF tasks were executed first passively by the robot (10 $\mathrm{min}$ ) to familiarize the patients to the robot and to warm up the muscles. Subsequently, the subjects actively performed the tasks by using EMG-driven control (20 min). The goal was to achieve a CR of 40 repetitions (5 repetitions $\times 4$ singleDoF tasks $\times 2$ directions) during $20 \mathrm{~min}$. At the beginning of each trial, the subject was positioned at the starting position for the respective task (Table 5). Forty repetitions was the number of repetitions that the healthy subjects were able to perform in 20 min without strong fatigue, and this was therefore adopted as the desired goal for the patients due to limited therapy duration. In the case that a patient reached 40 repetitions under $20 \mathrm{~min}$, the session was stopped. The sequence of tasks was randomized daily. 
The subject was instructed to make an effort to move the robot towards the final position $\left(\vec{P}_{\text {Final }}\right)$. Doing this, he/she activated the triggering muscle. The subject was then expected to monitor the visual feedback (Figure 2(c1)) and modulate the effort so that the light blue square $\left(A_{E M G}^{k}(m)\right)$ moved out of the red zone in the direction of the green arrow, surpassing the dashed black line $\left(T h_{E M G}^{k}\right)$. As long as the subject maintained the light blue square above the threshold line, the robot assisted the movement towards the final position. Once activated, the robot moved at the maximum speed ( $5 \%$ ). The total duration of a repetition, and therefore the number of completed exercises (task completion rate) within the session time for EMG control (20 min) depended on how well the subject was able to maintain the muscle activation above the threshold.

The EMG signals were displayed continuously on the computer screen to the physiotherapist during the session, so that hypotonic and compensatory muscle activity were immediately detected and corrected by the physiotherapist. The EMG signals were therefore used as an additional tool for the physiotherapists in order to enhance selective control of proximal shoulder muscles.

\subsubsection{Data Analysis}

The recorded EMG signals were manually inspected in order to remove those task repetitions with evident artifacts due to the electrode displacements or accidental contacts with the robotic parts.

\subsubsection{Evaluation of the Robot Compliant Operation}

For the single-DoF target-tracking tasks, the average level of muscle activation during the task was estimated as the mean value of the Root Mean Square (RMS) computed over the data windows of $128 \mathrm{~ms}$ with $50 \mathrm{~ms}$ of overlap. The mean RMS 
was normalized to the maximum mean RMS for the specific muscle across tasks and conditions which was obtained consistently in C5.

For the EIGHT task, the linear EMG envelope was plotted in order to assess the modulation of the EMG activation during the entire execution of the task. The envelopes were normalized to the maximum of the envelope for the specific muscle across tasks and conditions.

\subsubsection{Clinical Evaluation with EMG-driven Control}

The activation profiles for the triggering muscles per task, $A_{E M G}^{k}$, were normalized by the MVC of the triggering muscle obtained during the task calibration $\left(M V C_{E M G}^{K}\right)$ in order to compare the EMG activity of triggering muscles taskwise among subjects.

The activation profiles for the scapular muscles per task, $A_{E M G}^{\text {teres,infraspinatus }}$, were normalized using MVCs $\left(M V C_{E M G}^{\text {teres }}\right.$ and $\left.M V C_{E M G}^{\text {infraspinatus }}\right)$ obtained during the IE task. The infraspinatus activity was computed for the movements HORZ ABD, ABD and FLEX, while the activity of the teres was determined for HORZ ADD, ADD and EXT, because these muscles are mainly active during the respective tasks.

The maximum values of the activation profiles for the triggering and scapular muscles $\left(\max \left(A_{E M G}^{k}\right)\right)$ for each task and subject group were calculated as the median of the maximum activation values for each trial.

To assess the performance in completing the exercise, the CR was noted for each subject in each task, by counting the number of exercises in which the subjects reached the final position using active myocontrol. This does not include the repetitions in which the time out period expired, and the robot therefore completed the movement on behalf of the passive subject.

The median completion rate over all days and the patients' subjective evaluation on the ease of use of EMG control for group A were compared with group B using the 
Mann-Whitney $U$ Test. For each group, the comparison of CR among days was done using the Friedman test. The comparison of the maximum values of the activation profiles $\max \left(A_{E M G}^{k}\right)$ among groups was done using Kruskal-Wallis ANOVA by Ranks and Median Test, followed by post-hoc multiple comparison of mean ranks using Dunn test. Nonparametric tests were used because the data distributions were not normal, as determined by the Shapiro-Wilk Test. The statistical differences and normality rejections was tested at a significance level of $p<0.05$. In the texts, plots and tables the level of statistical significance is indicated as follows: ' $*$ ' for $p<0.05$, '**' for $p<0.01$, '***' for $p<0.001$ and '****' for $p<0.0001$. The data is represented in boxplots with the median, First (Q1), Third Quartile (Q3), min/max values and outliers. The min/max values are the values whose distance from Q1 downwards or Q3 upwards does not exceed 1.5 times the box height. The box height is the IQrange (Q3-Q1). The outliers are the values whose distance from Q1 downwards or Q3 upwards exceeds 1.5 times the box height. The statistical tests were performed in STATISTICA v12 (StatSoft, USA).

\subsubsection{Results}

\subsubsection{Evaluation of the Robot Compliant Operation}

The mean activity of the primary agonist muscles across different conditions for the selected single-DoF target-tracking tasks is displayed in Figure 6. The activity of the target muscles was modulated as the task and robot configuration parameters were changed, and this modulation was well controlled for all tested subjects, i.e., the primary agonist muscle was recruited through a range of activity levels by changing the conditions from $\mathrm{C} 1$ to $\mathrm{C} 6$.

Increasing the maximum torque during both slow (C1 vs C3 in Figure 6(a-c)) and fast movements (C2 vs C4 in Figure $6(\mathrm{a}, \mathrm{b})$ as well as increasing the movement speed in 
both soft (C1 vs C2 in Figure $6(a, b))$ and stiff modes (C3 vs C4 in Figure 6(a, b)) resulted in the higher values of the mean muscle activity over the repetitions. The muscle activity increased when the subject was instructed to stay at the target for a longer time during fast movements and stiff mode (C4 vs C5 in Figure 6(a,b)) and slow movements and soft mode (C1 vs C6 in Figure 6(c)) due to a prolonged buildup of the robot resistive torques. Overall, the highest mean RMS value was registered when the holding time, movement speed and the robot maximum force were set to the high values (condition C5 in Figure 6(a-c)). Therefore, in all the conditions, the selective activation of muscles was achieved by manipulating the robot configuration parameters. The primary agonists were the most active as the robot provided gravity compensation during the movement.

A similar modulation of EMG activity was observed during the EIGHT task. Figure 7 shows the activation of the pectoralis major and teres major in one representative subject while traversing the trajectory, when the robot was in the soft mode (Figure $7(\mathrm{a}, \mathrm{c}))$ and when the stiffness was increased (i.e., stiff mode), but only along the HORZ ADD/ABD DoF (Figure 7(b,d)). Pectoralis major (Figure 7(a)) was most active while moving along the diagonal parts of the trajectory, i.e., from the center
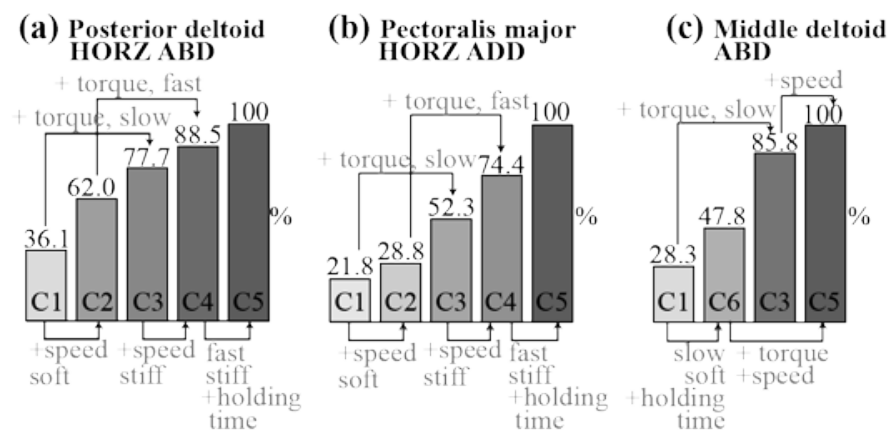

Figure 6: Modulation of EMG activity of triggering muscles during the target-tracking singleDoF by changing task and configuration parameters. Normalized mean value of the RMS of the EMG-signals recorded from (a) Posterior Deltoid of subject 1 during horizontal abduction (HORZ ABD), (b) Pectoralis Major of subject 2 during horizontal adduction (HORZ ADD) and (c) Middle Deltoid of subject 3 during shoulder abduction (ABD) across the condition $\mathrm{Ci}, \mathrm{i}=1, \ldots, 6$ of Table II. The terms (soft, stiff) and (slow, fast) are descriptive names for the (minimum, maximum) values in the columns "Maximum torque around the DoF" and "Speed of Movement" in Table II, respectively. 
upwards, which corresponds to a combination of flexion and horizontal adduction, and from the center downwards, corresponding to a combination of extension and horizontal adduction. During the horizontal segments of the trajectory (top and bottom arches), which represent horizontal abduction, the muscle was almost silent. This pattern of activity agrees with its biomechanical function [59], i.e. it is a flexor and horizontal adductor of the shoulder.

When the stiffness was increased selectively along HORZ ADD/ABD, the muscle reproduced the same overall pattern, but with a higher level of activation. Importantly, the increase in activation was observed only in those muscles involved in the HORZ ADD/ABD such as Pectoralis Major (Figure 7(b)), Anterior and Posterior Deltoids. Indeed, as shown in Figure $7(\mathrm{c}, \mathrm{d})$, no major EMG modulation was observed in the teres major between the two conditions since its principal actions are shoulder adduction and shoulder internal rotation [59]. Therefore, by manipulating the robot configuration parameters, the selective recruitment of muscles was achieved even during the exercise that included simultaneous activation of two DoF.

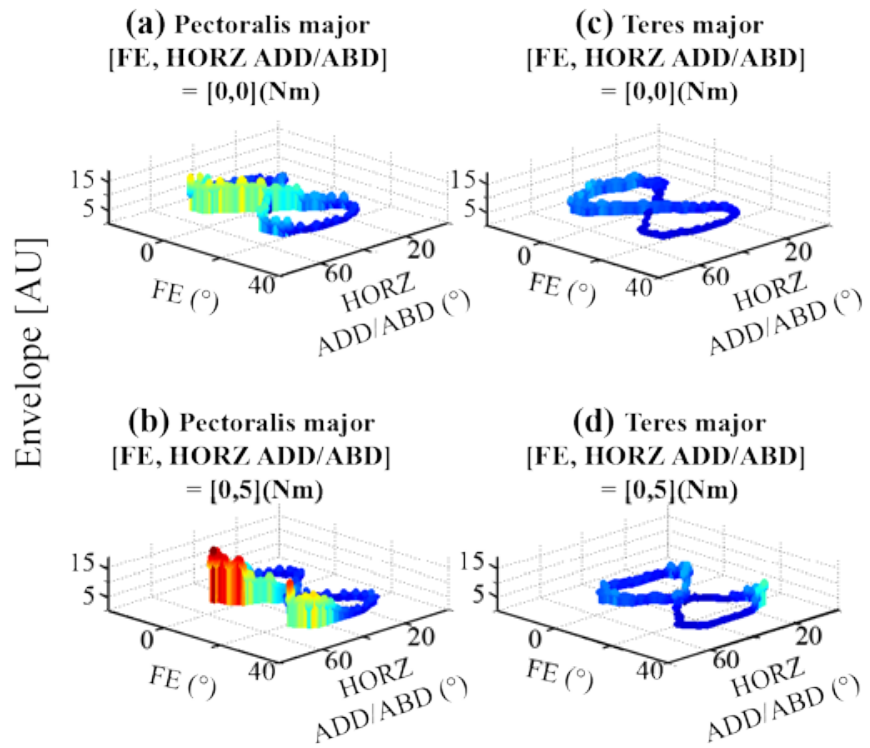

Figure 7: Modulation of EMG activity of pectoralis major and teres major during an EIGHT task when modifying the parameter maximum torque along HORZ ADDIABD in one representative subject. Linear envelopes of pectoralis major $(a, b)$ and teres major $(c, d)$ in both soft $(a, c)$ and stiff (b, d) mode, respectively. The annotation [FE, HORZ ABDIADD] indicates the maximum torque setting around the FE and HORZ ADDIABD DoF, respectively. 


\subsubsection{Clinical Evaluation with EMG-driven Control}

The recorded signals for the movement along IE DoF for a representative patient

with a mild impairment are illustrated in Figure 8 to demonstrate the operation of the robot. As long as the momentary EMG activation level of the teres major muscle was over the threshold ( $T h_{E M G, \text { teres }}=9 \mathrm{mV}$, see green signals in Figure 8(a)), the robot actively assisted the patient until completing the task of internal rotation (I), i.e., DoF3 changed from $-8.4^{\circ}$ to $37.6^{\circ}$ (Figure $8(b, c)$ ). Similarly, while the infraspinatus muscle was over the threshold $\left(T h_{E M G \text {,infraspinatus }}=17.6 \mathrm{mV}\right.$, see light blue signals in Figure 8(a)), the robot provided assistance during external rotation (E), i.e., the DoF3

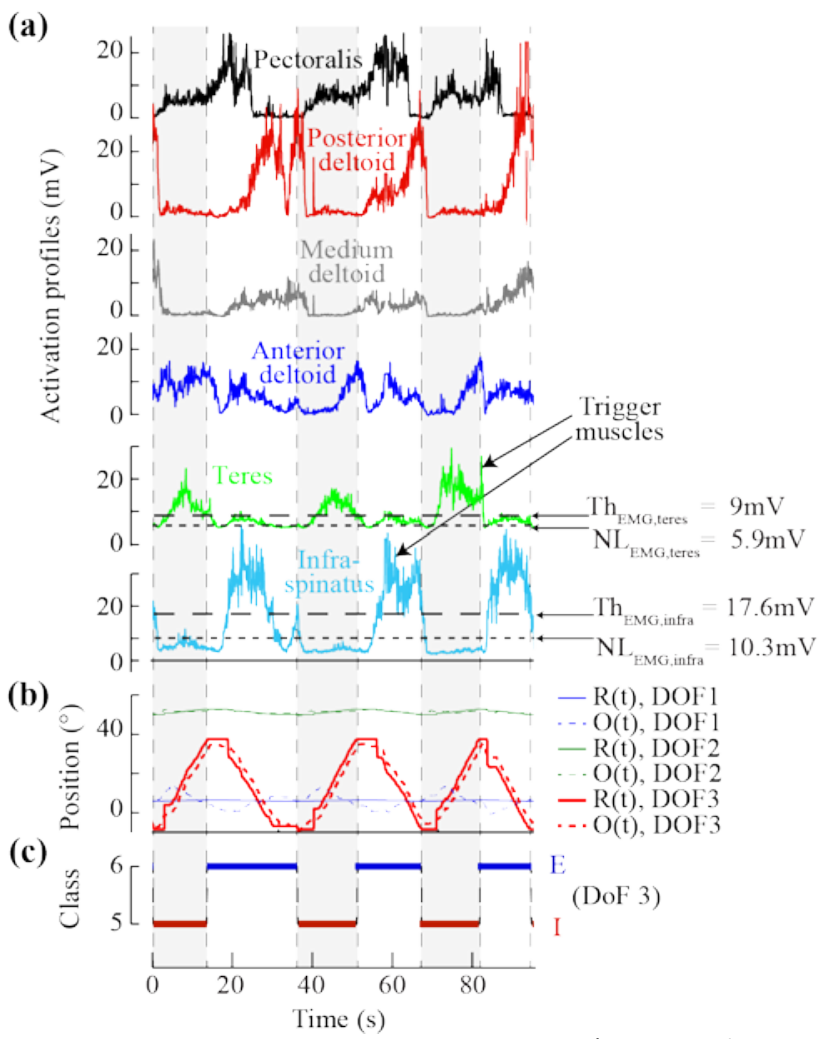

Figure 8: Activation profile of six shoulder muscles, $A_{E M G}^{1}, \ldots A_{E M G}^{6}$ (a) and robot position (b) when a representative patient with a mild impairment was performing the exercise of internal/external rotation, DoF3 (c) using myoelectric control. The $A_{E M G}^{k}$ were the activation profiles determined and saved online during the session. The external rotation corresponded to a displacement of 46 degrees (from $37.6^{\circ}$ to $-8.4^{\circ}$ ) and the internal rotation corresponded to the reverse movement. The patient moved mostly along DoF3 (task direction), but the movement was not entirely planar, since there were some excursions along DoF1 and DoF2 (approx. $10^{\circ}$ and $2.9^{\circ}$, respectively). The teres major and infraspinatus muscles were the triggering muscles for the internal and the external rotation, respectively. The activity of these muscles was strong enough to drive the robot progressively through the task without the robot's passive assistance. In (b) $R(t)$ is the reference position which the patient should follow and $O(t)$ is the patient's generated trajectory. 
changed from $37.6^{\circ}$ back to $-8.4^{\circ}$ (Figure $8(b, c)$ ). This patient tended to move the robot also around DoF1 while rotating the shoulder, but the robot limited the excursions along this DoF since it was configured to be stiff around DoF1 (maximum restorative torques set to $20 \mathrm{Nm}$ ). The patient had no problem in activating the triggering muscles consistently above the threshold and completing the tasks without the robot assistance (Figure 8(a)).

Summary results of the CR for the two patient groups over consecutive days are plotted in Figure 9. All healthy subjects consistently reached the maximum number of repetitions (40) in each session, and the results for them are therefore not shown. The mild impairment group B performed significantly better $(p<0.0001)$ than the severe-to-moderate impairment group A over all days. The overall median CR was 39 task repetitions, $\mathrm{Q} 3=40, \mathrm{Q} 1=32.5$ for group $\mathrm{B}$ and the overall median $\mathrm{CR}$ was 24 task repetitions, $\mathrm{Q} 3=29, \mathrm{Q} 1=14$ for group $\mathrm{A}$. Group $\mathrm{A}$ increased the

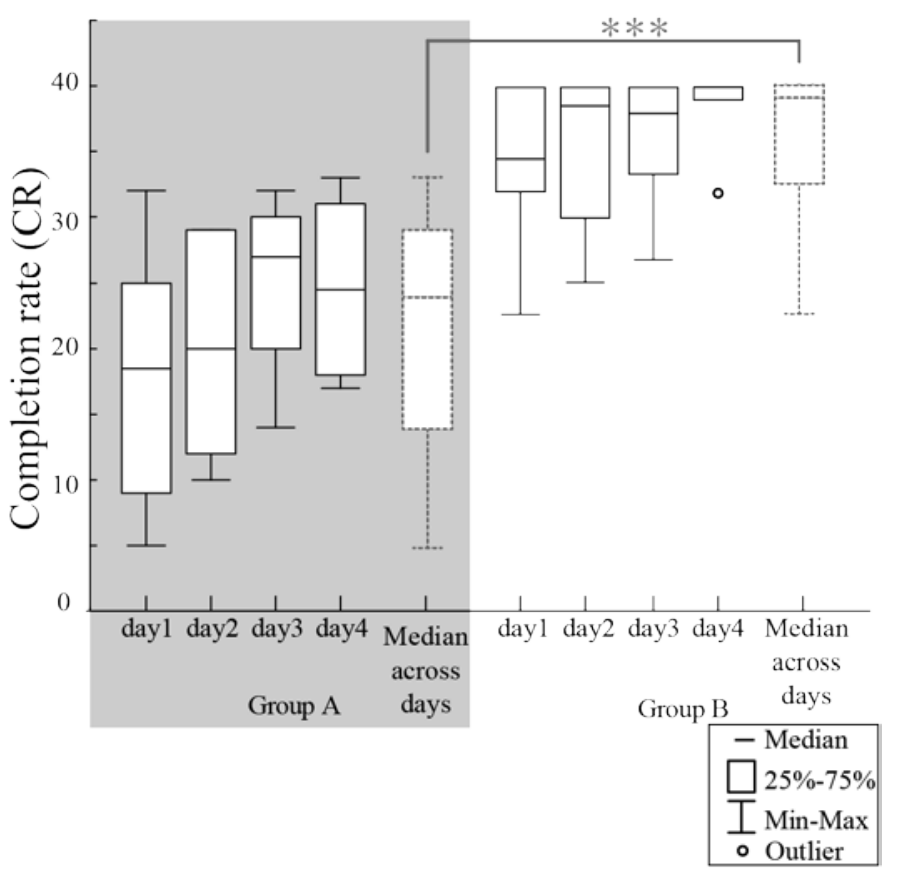

Figure 9: Completion rate (CR) for group $A$ and group $B$ using the EMG-driven control system over consecutive days (black boxplots) and median completion rate (CR) for group $A$ and group B over all days (dashed grey boxplots). The target CR per session for each control modality was 40 repetitions ( 5 repetitions $\times 4$ single-DoF tasks $\times 2$ directions). There was a tendency towards a higher number of repetitions over consecutive days for both groups, but no significant difference was found. Group B executed a significantly higher number of repetitions over all days ( $p<0.0001$, grey asterisks and bar) in comparison to group $A$. 
performance over time. The median CR was 18, 20, 27, and 24 from day 1 to day 4, respectively. Group B exhibited a similar trend, increasing the median CR from 34 on day 1 to 38, 38, and 40 on the remaining days. However, the differences over consecutive days were not statistically significant for any of the groups.

Figure 10 compares the muscle activation profiles $\left(A_{E M G}^{k}\right)$ between the two patients with mild and strong impairment, and a heathy subject. The stroke patients exhibited a reduced and uncoordinated muscle activity with respect to the healthy subject. During HORZ ADD/ABD, the healthy subject (Figure 10(a)) generated well-defined bursts of activity in the triggering muscles, i.e., pectoralis and posterior deltoid. The bursts were above the threshold and out of phase, to drive the robot along the task DoF in both directions. The activity of the other two deltoid muscles and infraspinatus were in phase with the posterior deltoid, while the teres major supported the shoulder with a consistent activation during the entire movement in both directions.

During the same movement, the representative mild patient (Figure 10(b)) exhibited visible bursts of activity of the pectoralis muscle, although the activation was barely crossing the threshold level. The bursts in the other triggering muscle (posterior deltoid) were not that clearly defined which also holds for the other two deltoids and infraspinatus. The mild patient accomplished 36 repetitions in the session and did not trigger the time out. The representative severe-to-moderate patient generated visible bursts of activity in the pectoralis muscle, but the other triggering and supporting muscles were almost silent, with some spontaneous activation during the tasks (Figure 10(c)). The severe patient accomplished only 8 repetitions in the session and triggered the time out 32 times, activating the robot to take over and passively move the arm to the end of the trajectory. 


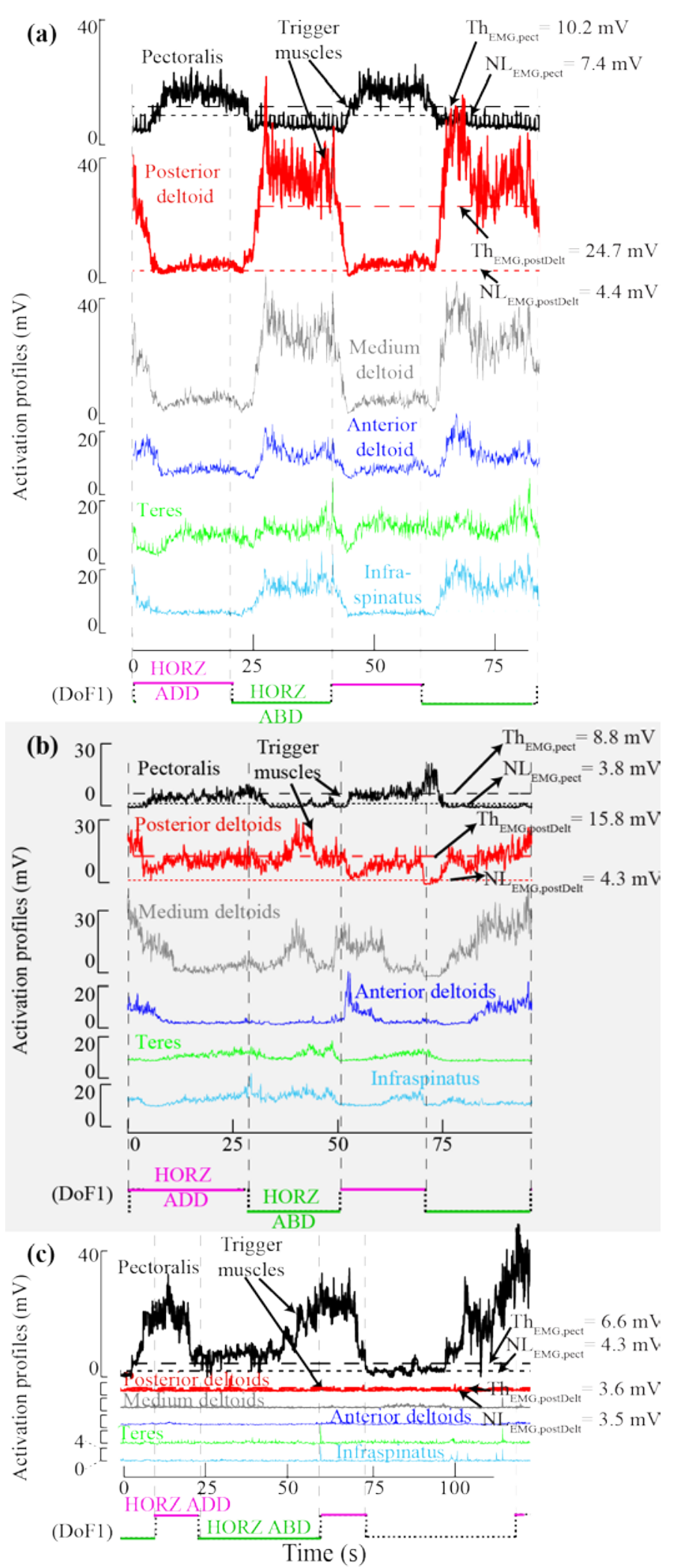

Figure 10: Activation profiles of six muscles during the execution of a HORZ ADDIABD for a representative healthy subject (a), a mild patient (b) and a severe-to-moderate patient (c). The threshold values for the triggering muscles corresponded to the $35 \% \mathrm{MVC}$ and the baseline corresponded to the maximum EMG activity at rest. Note the similar threshold values for the representative healthy subject and mild patient. While these values for the representative severe patient were markedly lower, especially for the posterior deltoid. The EMG activity of the pectoralis was similarly strong for all subjects (See also table VI). Note that the axes range for each plot is different, e.g. for the infraspinatus, teres and deltoid muscles of the severe subjects, the range goes from [0-4] $\mathrm{mV}$.

The maximum activity of the rotator cuff muscles (Table 6) for group A was 
Table 6: maximum values of emg activity of the rotator cuff muscles for each task (median; mean \pm std)

\begin{tabular}{|c|c|c|c|c|c|c|c|c|}
\hline Task & \multicolumn{2}{|c|}{ Exercise 1} & \multicolumn{2}{|c|}{ Exercise 2} & \multicolumn{2}{|c|}{ Exercise 3} & \multicolumn{2}{|c|}{ Exercise 4} \\
\hline & $\begin{array}{l}\text { HORZ } \\
\text { ADD }\end{array}$ & $\begin{array}{l}\text { HORZ } \\
\text { ABD }\end{array}$ & $\mathrm{ADD}$ & $\mathrm{ABD}$ & I & $\mathrm{E}$ & Ext & Flex \\
\hline $\begin{array}{l}\text { Trigger } \\
\text { muscle }\end{array}$ & Teres & $\begin{array}{l}\text { Infra- } \\
\text { spinatus }\end{array}$ & Teres & $\begin{array}{l}\text { Infra- } \\
\text { spinatus }\end{array}$ & Teres & $\begin{array}{l}\text { Infra- } \\
\text { spinatus }\end{array}$ & Teres & $\begin{array}{l}\text { Infra- } \\
\text { spinatus }\end{array}$ \\
\hline Group A & $\begin{array}{c}4.3 ; \\
4.2 \pm 1.4^{+}\end{array}$ & $\begin{array}{c}5.7 ; \\
6.7 \pm 3.5\end{array}$ & $\begin{array}{c}3.9 ; \\
4.8 \pm 2.5^{+}\end{array}$ & $\begin{array}{c}5.4 ; \\
5.7 \pm 2.7^{+}\end{array}$ & $\begin{array}{c}3.9 ; \\
4.3 \pm 1.7 *\end{array}$ & $\begin{array}{c}6.9 \\
7.7 \pm 4.2^{+}\end{array}$ & $\begin{array}{c}4.4 ; \\
6.3 \pm 4.6^{+}\end{array}$ & $\begin{array}{c}4.8 ; \\
4.8 \pm 1.7^{+}\end{array}$ \\
\hline Group B & $\begin{array}{c}7.5 ; \\
9.3 \pm 6.3\end{array}$ & $\begin{array}{c}7 ; \\
6.2 \pm 3.2\end{array}$ & $\begin{array}{c}10.5 \\
12.4 \pm 6\end{array}$ & $\begin{array}{c}6.2 ; \\
7.3 \pm 3.8\end{array}$ & $\begin{array}{c}16.9 ; \\
15.4 \pm 3.6\end{array}$ & $\begin{array}{c}15.7 ; \\
13.2 \pm 4.9\end{array}$ & $\begin{array}{c}11.4 ; \\
13.4 \pm 4.6\end{array}$ & $\begin{array}{c}3.4 ; \\
3.7 \pm 1.3^{\circ}\end{array}$ \\
\hline $\begin{array}{l}\text { Healthy } \\
\text { subjects }\end{array}$ & $\begin{array}{c}18.7 \\
17 \pm 8.7\end{array}$ & $\begin{array}{c}15.6 ; \\
14.9 \pm 8.9\end{array}$ & $\begin{array}{c}16.1 ; \\
19 \pm 9.4\end{array}$ & $\begin{array}{c}13.3 ; \\
12.7 \pm 3.8\end{array}$ & $\begin{array}{c}20.4 ; \\
25.5 \pm 17.1\end{array}$ & $\begin{array}{c}21.8 ; \\
23.1 \pm 6\end{array}$ & $\begin{array}{c}18.8 ; \\
20.9 \pm 7.2\end{array}$ & $\begin{array}{c}23.8 ; \\
22.8 \pm 13.9\end{array}$ \\
\hline
\end{tabular}

The values are in \%. An asterisk (*) or a degree symbol $(+)$ for group A means that the values of this group significantly differed (p<0.05) from the values of group B and the healthy subjects or just from the values of the healthy subjects, respectively. A degree symbol $\left({ }^{\circ}\right)$ for group B means that this group significantly differed $(\mathrm{p}<0.05)$ from the values of the healthy subjects.

significantly reduced $(p<0.05)$ in comparison to the healthy subjects during all tasks

except in HORZ ABD for the infraspinatus. The median values for the rotator cuff activation levels were higher in group $B$ compared to $A$, but not significant differences were found except for the teres activation in the internal rotation. Contrarily, there were no significant differences in the rotator cuff activations between group $B$ and the healthy subjects, except for the infraspinatus in the flexion. The maximum activation values of the triggering muscles (Table 7 ) significantly differed between the healthy subjects and the group A $(p<0.05)$ for all tasks except for the pectoralis activity in HORZ ADD. Contrarily, there were no significant differences between group B and the healthy subjects. Finally, the median values for the rotator cuff activation levels were higher in group B compared to A, but not significant differences were found except for the teres muscle in ADD and internal rotation and posterior deltoids in the extension.

Table 7: maximum values of emg activity of the triggering muscles for each task (median; mean \pm std)

\begin{tabular}{|c|c|c|c|c|c|c|c|c|}
\hline \multirow[t]{2}{*}{ Task } & \multicolumn{2}{|c|}{ Exercise 1} & \multicolumn{2}{|c|}{ Exercise 2} & \multicolumn{2}{|c|}{ Exercise 3} & \multicolumn{2}{|c|}{ Exercise 4} \\
\hline & $\begin{array}{l}\text { HORZ } \\
\text { ADD }\end{array}$ & HORZ ABD & ADD & $\mathrm{ABD}$ & I & $E$ & Ext & Flex \\
\hline $\begin{array}{l}\text { Trigger } \\
\text { muscle }\end{array}$ & Pectoralis & $\begin{array}{c}\text { Posterior } \\
\text { deltoid }\end{array}$ & Teres & $\begin{array}{c}\text { Medidum } \\
\text { deltoid }\end{array}$ & Teres & $\begin{array}{c}\text { Infra- } \\
\text { spinatus }\end{array}$ & $\begin{array}{c}\text { Posterior } \\
\text { deltoid }\end{array}$ & $\begin{array}{c}\text { Anterior } \\
\text { deltoid }\end{array}$ \\
\hline Group A & $\begin{array}{c}9 ; \\
11.2 \pm 7.7\end{array}$ & $\begin{array}{c}8.1 ; \\
7.7 \pm 4.3^{+}\end{array}$ & $\begin{array}{c}3.6 ; \\
3.9 \pm 1.2^{*}\end{array}$ & $\begin{array}{c}5.8 \\
5.8 \pm 3.3^{+}\end{array}$ & $(/)$ & $(/)$ & $\begin{array}{c}6.7 ; \\
6.6 \pm 2.4^{*}\end{array}$ & $\begin{array}{c}7.4 ; \\
7.2 \pm 4.4^{+}\end{array}$ \\
\hline Group B & $\begin{array}{c}18.3 ; \\
17.1 \pm 7.2\end{array}$ & $\begin{array}{c}13.1 ; \\
12.7 \pm 3\end{array}$ & $\begin{array}{c}10.5 ; \\
13.4 \pm 6.2\end{array}$ & $\begin{array}{c}11.3 ; \\
11.1 \pm 2.2\end{array}$ & $(/)$ & $(/)$ & $\begin{array}{c}13.3 ; \\
14.4 \pm 3.8\end{array}$ & $\begin{array}{c}12.3 ; \\
14 \pm 6.1\end{array}$ \\
\hline $\begin{array}{l}\text { Healthy } \\
\text { subjects }\end{array}$ & $\begin{array}{c}17.9 ; \\
18.9 \pm 6.5\end{array}$ & $\begin{array}{c}15.1 ; \\
18.8 \pm 11.6\end{array}$ & $\begin{array}{c}14.8 ; \\
16.9 \pm 10.3\end{array}$ & $\begin{array}{c}13.9 ; \\
13.5 \pm 4.2\end{array}$ & $(/)$ & $(/)$ & $\begin{array}{c}12.7 ; \\
16 \pm 8.8\end{array}$ & $\begin{array}{c}15.1 ; \\
14.9 \pm 4.3\end{array}$ \\
\hline
\end{tabular}

The values are in \%. (/) The values for the Infraspinatus and Teres for exercise 3 here are the same as in the previous table because these muscles were the triggering muscles for this task. An asterisk $\left(^{*}\right)$ or a plus symbol $(+)$ for group A means that the values of this group significantly differed $(\mathrm{p}<0.05)$ from the values of group B and the healthy subjects or just from the values of the healthy subjects, respectively. 


\subsubsection{Discussion}

Rehabilitation robots can be effective for motor training since they can reproduce exercise protocols in a consistent manner, provide multi-modal feedback to the user (e.g. tactile, visual) and customize the interventions based on individual physical impairments for task-specific training [23]. A suitable robot for upper limb stroke rehabilitation should be comfortable, safe and easy to attach to the patient's arms. Moreover, the robot should also allow the interaction between the therapist and the patient during an exercise in order to allow the therapist to be an active supervisor of the training.

In this engineering study, the novel RehaArm robot for the rehabilitation of the shoulder in stroke patients has been introduced. Due to a pneumatic actuation, RehaArm is an inherently compliant robot. Therefore, it provides a soft contact with the patient, responding promptly to his/her movements and/or interventions from the therapist. The patient was comfortably seated on the robot chair and his/her arm was simply strapped to the robotic arm. Compliant operation and the slow movement make the robot a safe platform, which can be used to provide the therapy even in an early stage after stroke. The motor training with RehaARM allows for task-specific training of the shoulder, the first joint to be mobilized in the acute phase. The tasks were functional and permit the shoulder training for ADLs. For example, the shoulder flexion is a function required in lifting the arm for grasping an object.

The RehaARM system with myoelectric control permitted patients - even those severely affected - to understand how to initiate, continue and complete a motor task activating the primary muscle, and thus synergistic muscles for the execution of a certain task. The elbow and the hand joint were fixed and this facilitated the shoulder tasks. Even severely impaired patients were able to drive the robot into a movement while mild patients were able to actively perform brighter shoulder movements. 
Therefore, the RehaARM system can be used for-task specific training according to individual needs.

We demonstrated that RehaArm can be used to implement different exercise modalities such as compliance-based training, EMG-driven control or a combination of both. We performed a preliminary test in which different robot configuration settings (speed, stiffness), experimental task parameters (trajectory length, holding time), and experimental paradigms (single- and multi-DoF activation) have been tested in healthy subjects to investigate the properties and usability of the robotic device.

The results demonstrated that the motor response of the volunteers was selectively modulated and in a well-controlled manner by changing the experimental task and robot configuration parameters. The activity in the specific muscle (or muscle group) was adjusted gradually to a range of levels (Figure 6). Importantly, there is flexibility in implementing these adjustments; several parameters are available to modulate the muscle activation (see Table 2), and these can also be combined for the cumulative effect (and these can be also combined for the cumulative effect (as done in the single-DoF target-tracking task experiment). Therefore, the exercise can be adjusted according to the needs of an individual subject. For example, at the beginning of the training, the patient could start with a soft robot, small excursions and a touch and go single-DOF tracking task. Later on, as his/her status improves, the stiffness and/or trajectory length and/or time on target could be increased, posing a more challenging task to the subject. There is a strong evidence that adapting the task difficulty to the current status of the patient promotes the recovery [60]. In the multi-DoF movement (EIGHT task), the muscle groups that were active during movements along a certain DoF were additionally loaded by increasing the robot 
resistance selectively along that specific DoF (Figure 7). The parameters used to regulate the muscle activity during single-DoF movements can be applied in the same way in a multi-DoF exercise.

Finally, we have demonstrated that the active robot assistance triggered by the user using muscles signals (i.e. EMG-driven control) can be used for an experimental protocol in a clinical setup. Indeed, mild and severe-to-moderate impaired stroke patients were able to drive the robot by producing EMG activation in the primary agonist (triggering) muscle along the selected task, which resulted in smooth movement trajectories (Figure 8 and Figure 10). The mild and severe-to-moderate patients were able to use the EMG-driven control and performed better over the four consecutive days. The mild patients performed at day 4 similarly well (median CR 40) to the healthy subjects group who all executed 40 task repetitions in 20 min on the first day. The severe-to-moderate impairment group executed 27 and 24.5 (median) task repetitions on day 3 and day 4 , respectively. That is approximately $67 \%$ and $61 \%$ of the performance of the healthy subjects and mild patients. Both patient groups consistently improved over time. We used completion rate (CR) as a measure of performance. This measure reflects better voluntary control of the target muscles and a better understanding of using the system. This is not the result of only compensatory mechanisms because physiotherapists could adjust the level of difficulty of the training to the patient's ability to correct for compensation. The increment of $\mathrm{CR}$ which we obtained does not necessarily indicate clinical improvement. The length of the experiment was four days and this is too short time to achieve clinical improvements.

RehaArm with the EMG-driven control interface can therefore allow severe and moderately impaired patients to train arm motions, which are demanding and 
substantially above of what they could accomplish on their own (i.e., without gravity compensation and active assistance). Verbally, patients reported the motivating factor of using RehaArm in comparison to conventional therapy because they felt that they performed exercises more independently without the physiotherapist's physical assistance. This is a very motivating factor, especially for severe patients who can just be treated passively in conventional therapy [61]. Daily, the performance (CR) was shown to the patients and they were excited if they realized that they were able to do more. It is well known that concentration and engagement of the patient during the therapeutic treatment are important factors for recovery [62]. The single-DoF tasks were not perfectly planar and deviated depending on subjects' motor condition and anatomy. For example, the flexion task was not perfectly a flexion. It was rather a flexion with horizontal adduction and external rotation. This implies that while mainly activating DoF2, the other two DoFs were also slightly activated. This was the case also for the other tasks (e.g. Figure 8 for IE). Training of these shoulder single-DoF tasks is a condition for regaining proper arm function.

The choice of single-DoF exercises was a deliberate design choice for clinical evaluation of the robot. In neurodevelopmental approaches in physiotherapy, it is known that, particularly in severe cases, the best therapy choices in order to induce physiological reorganization after brain injury (true recovery) are the ones that patients can control actively, and are challenging enough. The aim is to avoid the adoption of compensatory motor strategies and shoulder pain with tasks that are too demanding to accomplish, due to the reduced functional ability [40], [63]. This choice had potential because we could involve a significant number of patients, including those more severely affected. 
Additionally, single-DoF exercises were more suitable for EMG-driven control with just one triggering muscle per task (Table 5). More complex movements, like the EIGHT tracking task, could have been confusing for patients, especially for those with more severely cognitive and concentration problems. Furthermore, tasks that are more complex require more advanced EMG-driven control algorithms relying on more muscles, a development that we consider as a future step. There was an attempt in this direction using pattern recognition for multi-DoF movements in the horizontal plane, but it was not practical [64]. Instead, it was suggested that a feedback of correct recruitment could be more practical.

Since the subject-robot interaction is soft, the execution of the task is not stereotyped, i.e., the subject is not forced to repeat consistently and precisely the same movements. Instead, trial-to-trial movements will exhibit a certain level of variability, which is also the characteristic of the physiological human motor control. For maximum flexibility, it would be also possible to set only the initial and final positions, letting the subject freely choose the most convenient approach trajectory. This work has been also motivated by the need to close the loop in rehabilitation robotics. As demonstrated in EMG-driven robot tasks, the robot can be driven by the subject by capturing and processing the EMG activity to detect the motion intention. Through closed loop control, sensory motor integration is achieved, and it is hypothesized that this can promote the relearning of the movement. Only few compliant robots have been presented up to now implementing this concept [30], [38], but there were no robots specifically targeting the shoulder movement.

The closed loop framework implemented in this study is flexible and can be used in many control scenarios, in addition to the context that has been shown here. The system allows for extensions and improvements to achieve an optimal outcome. For 
example, in the present work with EMG-driven control, we did not adapt the support given to the patients. It may prove more beneficial to develop dynamic training protocols (e.g. "assist as needed" [65]) that allow subjects to gradually integrate active support of the limb with control of voluntary movement, in a manner similar to partial body weight support gait training [66]. Higher completion rate occurred because the challenge level was low for some patients of the mildly impairment group. We set forty repetitions as the maximum amount of repetitions to be performed in $20 \mathrm{~min}$ in order to compare the performance between patient groups. Future development should consider more challenging tasks and adaptation of threshold levels according to patient's improvement due to therapy.

By using multiple EMG channels for control, the system has the potential to be extended to actuate multiple shoulder DoFs sequentially or simultaneously, based on the detection of the patient's intention [67]. The robot treatment was well accepted by all patients, this is a very important factor for the introduction of new rehabilitation technologies.

\subsection{Study 2: Efficacy of Torque Versus Myocontrol For Active, Robotic-Assisted Rehabilitation Of The Shoulder After Stroke: An Experimental Study Methods}

(This section is based on a published conference paper [2], "C) [2015] IEEE. Reproduced with permission. All rights reserved"; therefore text, results and graphics taken from this manuscript are not cited explicitly.

\subsubsection{Abstract}

Objective. This engineering study investigated whether torque or myoelectric control with EMG-thresholding is more practical during active, robotic-assisted therapy for the shoulder in a clinical setup. After showing the feasibility of using the RehaARM robot with closed-loop myoelectric control in study 1, the clinical and scientific 
question whether myoelectric control has a more efficient applicability than torque/force control of the RehaARM robot for achieving more intensive upper limb therapy arose. Approach. 10 healthy subjects used the RehaArm robot in one daily one-hour session while 23 hemiparetic stroke patients used the robot in four daily one-hour sessions. During each session, subjects repeatedly performed basic movements of the shoulder in passive and active mode. The tasks to be executed were the same shoulder movements of the previous study 1 . During the active mode (40 min in total), subjects were asked to complete 40 task repetitions (5 repetitions $\mathrm{x}$ 4 single-DoF tasks $\times 2$ directions) in $20 \mathrm{~min}$ for each modality, torque and myoelectric control. The number of movement repetitions achieved - task completion rate $(\mathrm{CR})$ - was tracked for each control modality as well as subjective opinion about the ease of use of each modality after each daily session. Main Results. Using myoelectric and torque control, the group of healthy subjects executed 40 task repetitions in $20 \mathrm{~min}$. The $\mathrm{CR}$ results over all days showed that the severe-tomoderate impairment group (group $A, n=13$ ) performed a significantly $(p<0.001)$ higher number of task repetitions in the given time with myoelectric control in comparison to torque control. For the mild impairment group (group $B, n=10$ ), the CR over all days was very similar for both control modalities, but their performance was more constant during the torque control (narrower IQ-range). Over time, group A performed tendentially better with both control modalities (higher median and Q1/Q3 values) and there was a significant increase between $\mathrm{CR}$ at day 1 and day 4 for myoelectric $(p<0.01)$ and torque control $(p<0.05)$. Over time, group B also performed tendentially better with both control modalities (higher median and Q1/Q3 values, and narrower IQ-ranges), but there was not a significant difference between days. According to the questionnaires, the severe-to-moderate impairment group $A$ 
considered myoelectric control significantly easier to use than torque control at day 1 and day $4(p<0.05)$ while the mild impairment group B considered both control modalities similarly easy to use. Group A and group B activated their upper limb muscles similarly during myoelectric and torque control with respect to the healthy subjects group (no significant difference between EMG-S and TORQUE-S was found). Significance. Myoelectric control with simple EMG-thresholding was more practical than torque control for robotic-assisted therapy. The stroke patients reported on the perceived ease of use of the robot. For severely-to-moderately impaired patients this was greater with myoelectric control than with torque control and this was a motivating factor. For mildly impaired patients this was the same for both types of control. There was a tendency to perform a higher number of task repetitions over time with both control modalities for both groups. This indicates that subjects learnt to adapt their motor control and use the robot with both control modalities, the myoelectric control being easier to use (higher $\mathrm{CR}$ and rating in questionnaires). The novel myoelectric control with simple-thresholding is practicable and neurophysiological because it can make a neurorehabilitation robot more responsive to subjects impairment (very small residual muscles signals can be reliably detected), and also allow the activation and coordination of muscles (muscle recruitment) of torque control.

\subsubsection{Methods}

In this study, 10 healthy volunteers (five male and five female subjects, $53.4 \pm 18.1$ yrs) and 23 hemiparetic stroke patients (characteristics in Table 8) meeting study inclusion criteria were included. The inclusion and exclusion criteria were the same as for study 1. The IT-NIHSS score was also considered for assessing the maintenance of residual voluntary motor activation necessary for driving the robot 
Table 8: patients characteristics for study $2(\mathrm{n}=23)$

Table 8: patients characteristics for study $2(\mathrm{n}=23)$
\begin{tabular}{|c|c|c|c|}
\hline & Frequency & median & Q1-Q3 \\
\hline Age & & 59.7 & $48.5-70.3$ \\
\hline Gender (M / F) & $15 / 8$ & & \\
\hline Months since stroke & & 5 & $3.5-8.7$ \\
\hline FMA-UE ( $\leq \mathbf{3 8} / \mathbf{3 8 )}$ & $13 / 10$ & & \\
\hline
\end{tabular}

${ }^{*} \mathrm{Q} 1$ and Q3 are the first and third Quartile.

with myoelectric and torque control. The setting of the robot mechanically (arm length) and via software (max ROM) for adjusting the robot to the patient was done as in study 1. In addition, the parameters for the closed-loop control (increment step $\vec{d}_{i n c}$ and path tolerance $\vec{\Delta}_{p a t h}$ for myoelectric and torque control in Figure 3(a) and (b), respectively) and maximum torque values were set to the same values as for study 1 (section 3.1.2.2). The EMG baseline and MVC value for determining the threshold value in myoelectric control were obtained individually at each trial and session as done in study 1 (section 3.1.2.2).

The threshold for torque control was set individually by the physiotherapist at session 1 and it was adjusted if necessary in the following sessions. The physiotherapist took care that the torque threshold was high enough to make the task challenging yet moderate in order to avoid strong fatigue. In addition, for patients with severe impairments the torque threshold was set to the minimum settable torque values 1 $\mathrm{Nm}, 0.5 \mathrm{Nm}$ and $1 \mathrm{Nm}$ for DoF1, DoF2 and DoF3, respectively (section 2.2.4). This necessary setting for the correct function of the robot with torque control was a limiting factor for more severely impaired patients because they were often unable to exert enough residual force to surpass those torque values for some tasks, and therefore the robot was not activated.

Patients executed the protocol in four daily sessions. One session lasted for approximately $1 \mathrm{~h}$ including patient preparation. When possible, the four visits were scheduled to occur consecutively within a one-week window. The protocol comprised the same 4-single-DoF tasks of study 1 (Table 5 in section 3.1.2.2). During each 
daily session, the tasks were executed first passively by the robot (10 min), and afterwards subjects actively performed the tasks by using myoelectric (20 min) and then torque control (20 min), or viceversa (section 2.2.3 and section 2.2.4). The sequence of tasks and control modality were randomized for each session.

During the passive mode, subjects learnt the movements and got comfortable with the robot. During the active modes, the subject had to actively and continuously drive the robot to complete the tasks in closed-loop using visual feedback of his force (torque control) or muscles signals (myoelectric control). The robot assisted the patient if the torque or EMG activity surpassed an individually settable torque/EMG threshold value for each movement.

The instruction to control the robot with myoelectric control was the same as for study 1 (section 3.1.2.2). For torque control, the subject was also instructed to make an effort to move the robot towards the final position $\left(\vec{P}_{\text {Final }}\right)$ exerting residual force. The subject was then expected to monitor the visual feedback (Figure 2(c1)) and modulate the effort (torque) so that the light blue square $\left(T^{j}(m)\right)$ moved out of the red zone in the direction of the green arrow, surpassing the torque threshold for the corresponding task (dashed black line, $T h_{T O R Q U E}^{j}$ ), where $j=1, \ldots, 4$ represents each task. As long as the subject maintained the light blue square above the torque threshold line, the robot assisted the movement towards the final position. Once activated, the robot moved at the same maximum speed (5\%) as for myoelectric control.

The goal CR was 40 repetitions for each control modality during 20 min as in study 1. Healthy subjects were able to perform 40 repetitions with each modality in 20 min without strong fatigue, and this was therefore adopted as the desired goal for the patients due to limited therapy duration. The time out was also used as in study 1 . 
The total duration of a repetition, and therefore the number of completed exercises (CR) within the session time for EMG and torque control depended on how well the subject was able to maintain the muscle activation or torque above the threshold. After the first and fourth visit, two questions about the ease of use of each control modality were provided to the patients.

The questions in the questionnaire were:

1. How easy was to control the robot with torque (force of the arm)?

2. How easy was to control the robot using muscle signals?

Patients were provided with a visual analog scale displayed as a continuous line, ranging from 0 (difficult) to $10 \mathrm{~cm}$ (easy) to answer each of the questions. Each question was provided after each control modality. Then after completing the entire active part, an opportunity was given to review the answers. In this way, they completed both modalities and then confront them if they thought it was necessary. The EMG signals from the six triggering muscles were recorded at day 1,2 and 3 for all patients. At day 4, the EMG signals from 16 channels were recorded from a subgroup of 20 patients, and just the six signals of the triggering muscles for the rest of the patients $(n=3)$. For the healthy volunteers $(n=10)$, the EMG signals from 16 channels were recorded during the first only session (see section 2.2.2). The recorded EMG signals at day 4 were processed in order to obtain the synergistic modules for each task (i.e. muscle activations per task), and then calculate the overall muscle activation similarity between the healthy subjects and stroke patients when using each control modality (see section 2.3.3). 


\subsubsection{Data Analysis}

As for study 1, the recorded EMG signals were manually inspected in order to remove those task repetitions with evident artifacts due to the electrode displacements or accidental contacts with the robotic parts.

To assess the performance in completing the exercise, the CR was noted for each subject in each task and control modality per day, by counting the number of exercises in which the subjects reached the final position. This does not include the repetitions in which the time out period expired, and the robot therefore completed the movement on behalf of the passive subject.

The patients were classified in two groups as in study 1 . Therefore, there are three groups: the severe-to-moderate group A, the mild group B and the healthy subjects group.

For each group, the overall task completion rate between torque and myoelectric control were statistically compared using the Wilcoxon Matched-Pairs Test (within group comparison). For each group, the results of task completion rate between days were statistically compared using the Friedmann test and the Dunn's Post-hoc test. The results of the task completion rate of the patient groups were statistically compared using the Mann-Whitney U Test (between group analysis).

The results of questionnaires about the ease of use of the control modalities of the patient groups were compared using the Kruskalwallis test followed by a Dunn's post-hoc test (among group analysis). For each group, the ease of use of the control modalities at day 1 and day 4 and between day 1 and 4 were compared using the Wilcoxon Matched-Pairs Test (within group comparison).

The overall muscle activation similarity in each control modality of the subject groups was compared using the Mann-Whitney $U$ Test (between group analysis). The 
overall muscle activation similarity between control modalities per patient group was compared using the Wilcoxon Matched-Pairs Test (within group comparison).

As for study 1, nonparametric tests were used because the data distributions were not normal and the number of subjects was small for parametric tests, as determined by the Shapiro-Wilk Test. The statistical differences and normality rejections were tested at a significance level of $p<0.05$ as for study 1 . The data and statistical differences are indicated in the text, boxplots and tables as for study 1 . The statistical tests were done in the software IBM SPSS Statistics 22.

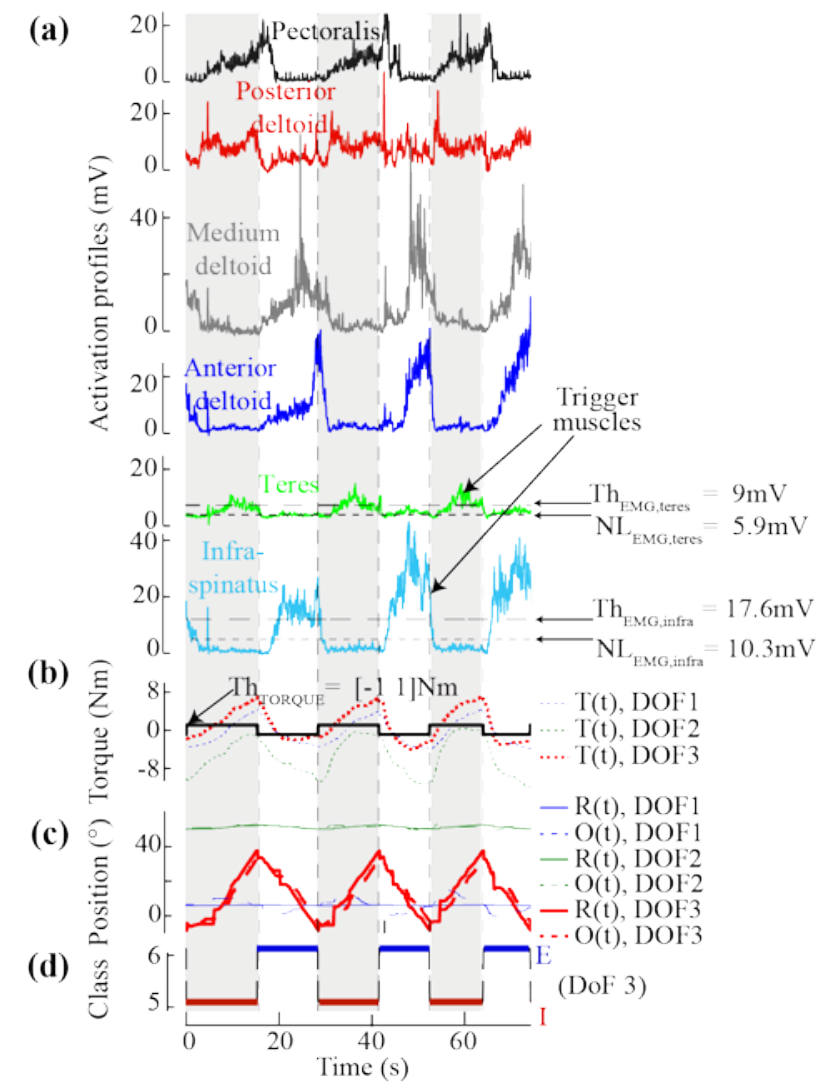

Figure 11: Activation profile of six shoulder muscles, $A_{E M G}^{1}, \ldots A_{E M G}^{6}(\mathrm{a})$, torque (b) and robot position (c) when the representative patient with a mild impairment was performing the exercise of internal/external rotation, DoF3 (d) using torque control. The $A_{E M G}^{k}$ were the activation profiles determined and saved online during the session. The external rotation corresponded to a displacement of 46 degrees (from $37.6^{\circ}$ to $-8.4^{\circ}$ ) and the internal rotation corresponded to the reverse movement. The patient moved mostly along DoF3 (task direction), but the movement was not entirely planar, since there were some excursions along DoF1 and DoF2 (approx. $13^{\circ}$ and $2.3^{\circ}$, respectively). The subject activated the robot when the torque value around DoF3 exceeded $1 \mathrm{Nm}$ in the corresponding direction. The patient's residual force was also strong enough to drive the robot progressively through the task without the robot's passive assistance. 


\subsubsection{Results}

The recorded signals for the movement along IE DoF for the representative patient with a mild impairment are illustrated in Figure 11 to demonstrate the operation of the robot. As long as the momentary torque threshold value around DoF3 in the direction of the of internal rotation (I) was over the threshold (1 Nm in Figure 11(b)), the robot actively assisted the patient until completing the task (I), i.e., DoF3 changed from $-8.4^{\circ}$ to $37.6^{\circ}$ (Figure 11 (c, d)). Similarly, while the torque level around DoF3 for of external rotation (E) exceeded the threshold (-1 Nm Figure 11 (b)), the robot provided assistance during (E), i.e., the DoF3 changed from $37.6^{\circ}$
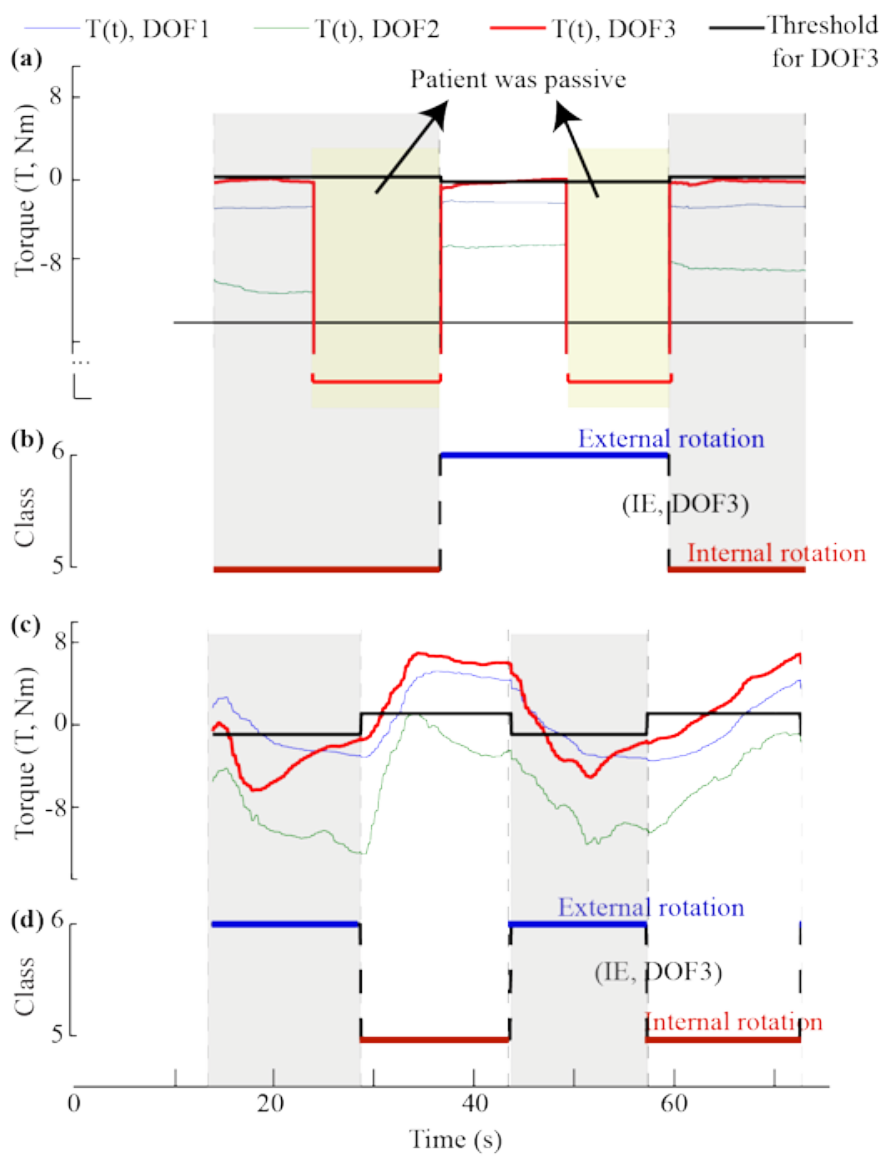

Figure 12: Torque (b) when the representative patient with severe-to-moderate impairment (c) and with mild impairment were performing the exercise of internal/external rotation, DoF3 (b, d) using torque control. For the severe-to-moderate patient, the external rotation corresponded to a displacement of 46 degrees (from $6^{\circ}$ to $-40.8^{\circ}$ ) and the internal rotation corresponded to the reverse movement. The subject activated the robot when the torque value around DoF3 exceeded $0.5 \mathrm{Nm}$ for (a) and $1 \mathrm{Nm}$ (b) in the corresponding direction. In (a), the patient's residual force was not strong enough to drive the robot. Therefore, the passive mode was activated by the physiotherapist in order to avoid excessive fatigue. For (c), the patient's residual force was strong enough to drive the robot progressively through the task without the robot's passive assistance. 
back to $-8.4^{\circ}$ (Figure $\left.11(\mathrm{c}, \mathrm{d})\right)$.

This patient also tended to move the robot around DoF1 while rotating the shoulder as with myoelectric control, but the excursions along this DoF were also limited (maximum restorative torques set to $20 \mathrm{Nm}$ ). Here again, this patient had no problem in applying residual force above the threshold level and completing the tasks without the robot assistance (Figure 11 (b)).

Patients from group A had more often difficulties in applying sufficient residual force to surpass the minimum torque threshold of the robot. For example, a representative patient from group A tried to apply residual force around DoF3 during the internal and external rotation to surpass the threshold of $0.5 \mathrm{Nm}$ for the I/E task (Figure 12 (a)). The subject had difficulties in performing the task; however, the physiotherapist waited for $14 \mathrm{~s}$ and allowed the patient try to perform the task. As the patient did not

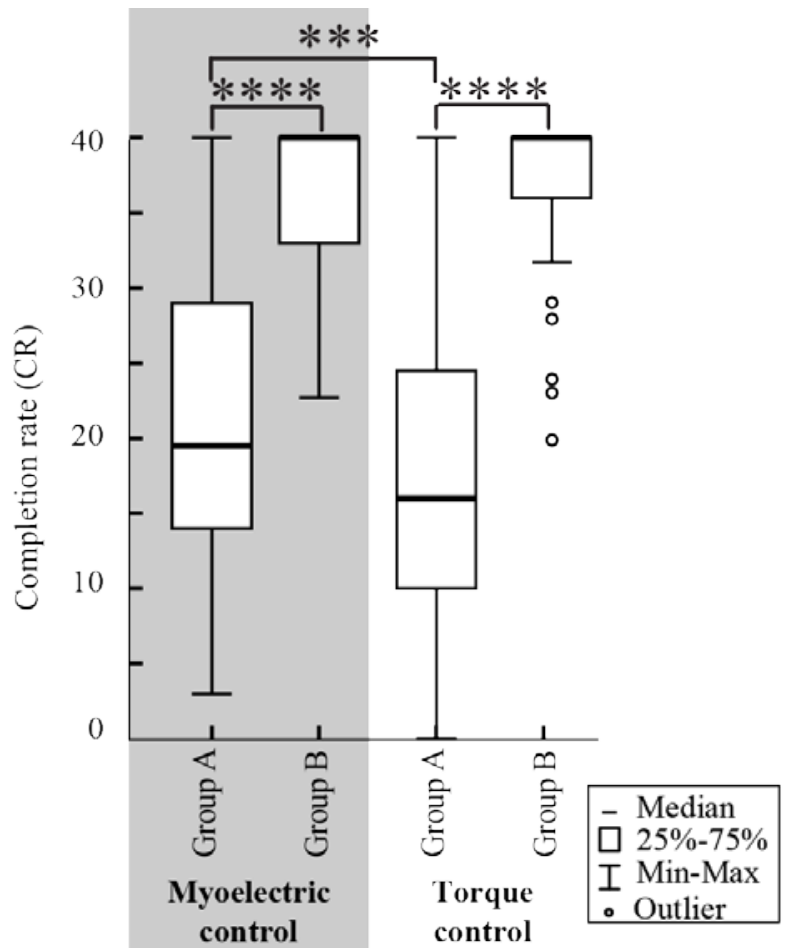

Figure 13: Task completion rate (CR) for group A and group B using the EMG-driven control system over all days (left grey shaded area) and task completion rate (CR) for group $A$ and group B using torque control over all days (right white area). The target CR per session for each control modality was 40 repetitions (5 repetitions $\times 4$ single-DoF tasks $\times 2$ directions). Group $B$ executed a significantly higher number of repetitions over all days using myoelectric and torque control ( $p<0.0001$, asterisks and bar) in comparison to the group $A$. 
manage to apply enough force, the passive mode was activated by the physiotherapist in order to avoid excessive patient's fatigue. In contrast, patients from group B had less difficulty in applying enough residual force and surpass the torque threshold to perform the tasks. For example, a representative subject from the mild patient group (Figure 12 (b)) applied enough force to surpass the torque threshold $(1 \mathrm{Nm})$ and perform the I/E rotation task without the robot's assistance.

Summary results of the CR for the two patient groups over all days are plotted in Figure 13. All healthy subjects consistently reached the maximum number of repetitions (40) in the daily session with both control modalities, and the results for them are therefore not shown. The CR results over all days showed that the severeto-moderate impairment group (group $A, n=13)$ performed a significant $(p<0.001)$ higher number of task repetitions in the given time with myoelectric control in comparison to torque control. The median CR over all days for myoelectric control was 18.5 task repetitions per daily session within 20 min with $\mathrm{Q} 3=28$ and $\mathrm{Q} 1=12.5$ and the median CR over all days for torque control was 15 repetitions with Q3 $=22.8$ and $\mathrm{Q} 1=10$. For the mild impairment (group $\mathrm{B}, \mathrm{n}=10$ ), the $\mathrm{CR}$ over all days was very similar for both control modalities, but their performance was more constant during the torque control (narrower IQ-range). For this group, the median CR over all days for myoelectric control was 40 task repetitions per daily session within 20 min with Q3 = 40 and Q1 = 33 and the median CR for torque control was 40 repetitions with Q3 = 40 and $\mathrm{Q} 1=35.5$.

The mild-impairment group B performed significantly better than the severe-tomoderate impairment group A over all days with myoelectric and torque control $(p<0.0001)$ 
Group A tended to increase the performance over time with myoelectric (left gray shaded area, Figure 14 (a)) and torque control (white area, Figure 14 (a)). From day 1 to day 4 , the median CR was $19,18,18$, and 20 for myoelectric control and 10, 15, 15, and 18 for torque control. There was a significant increase in performance at day 4 in comparison to day 1 for myoelectric $(p<0.01)$ and torque control $(p<0.05)$. Group B exhibited a similar trend (Figure 14 (b)), increasing the median CR from 33 on day 1 to 38.5 on day 2 and 40 on day 3 and 4 with myoelectric control and from 39.5 on day 1 to 40 on the remaining days with torque control. Group B used the robot very straightforward from the first day, and therefore the difference between days for group B was not significant.

According to the questionnaire (Figure 15), at day 1 and day 4 group A (light gray boxplots) considered myoelectric control significantly easier to use in comparison to

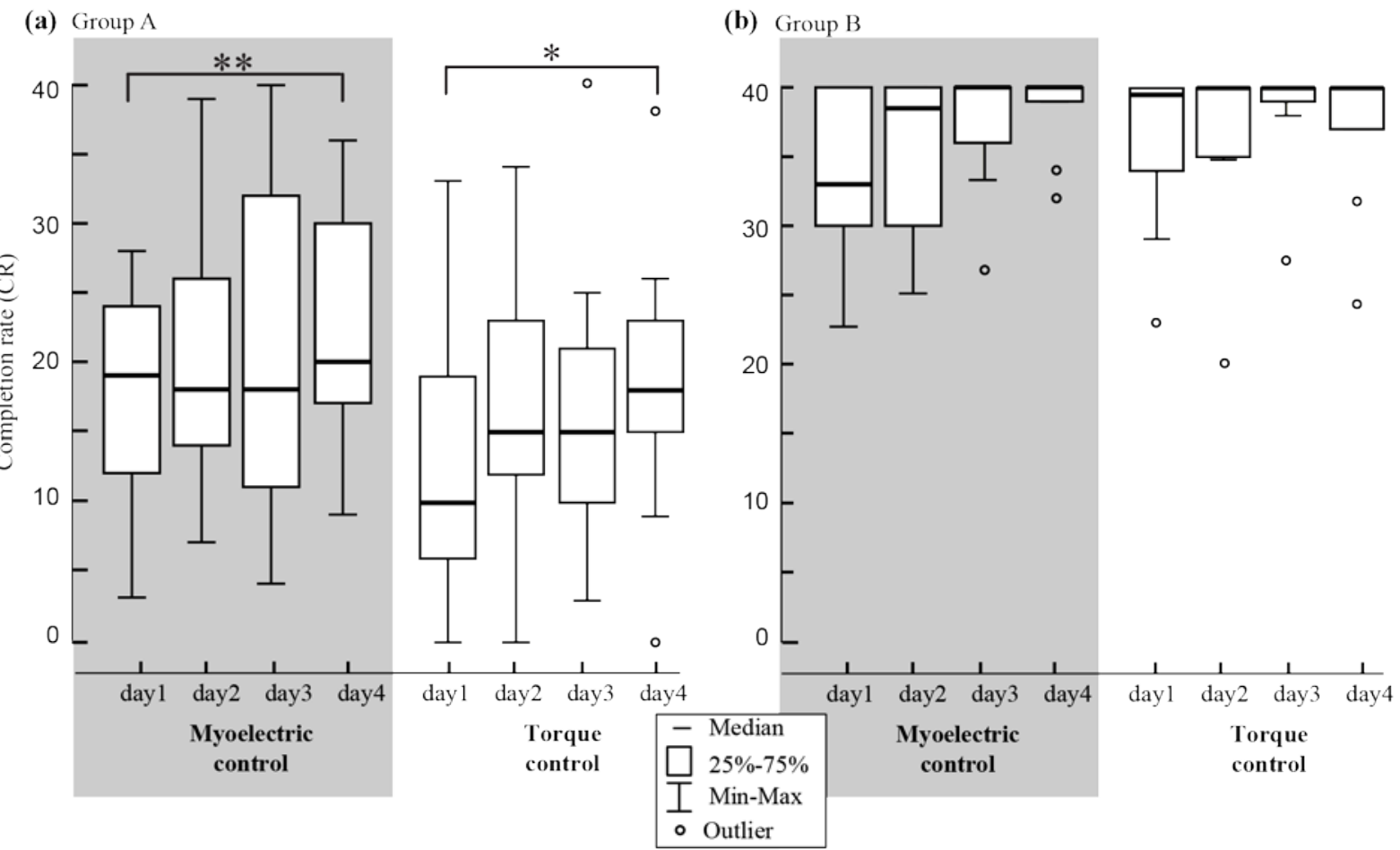

Figure 14: Completion rate (CR) for group A (a) and group B (b) using the EMG-driven (greyshaded areas) and torque control system (white areas) over consecutive days. The target CR per session for each control modality was 40 repetitions ( 5 repetitions $\times 4$ single-DoF tasks $\times 2$ directions). There was a tendency towards a higher number of repetitions over time for both groups. Group A performed better with torque and myoelectric control at day 1 in comparison to day $4(p<0.05)$. For group B, no significant difference was found. 
the torque control $(p<0.05)$ while group B (white boxplots) and the healthy subjects group (dark grey boxplots, only did one daily session) considered both types of control similarly ease to use (no significant differences were found).

At day 1 and day4, torque control was significantly easier to use for group $B$ in comparison to group A $(p<0.05)$. At day 1 , group B perceived that myoelectric control was significantly easier to use in comparison to group $A(p<0.05)$ while at day 4 both patient groups rated that myoelectric control was similarly ease to use (no significant difference was found). Healthy subjects used torque and myoelectric control significantly easier than group A at day $1(p<0.05)$ while in comparison to day 4 just torque control was significantly easier to use for the healthy subjects group $(p<0.05)$.

Subjects thought that they were able to use the system better after four sessions

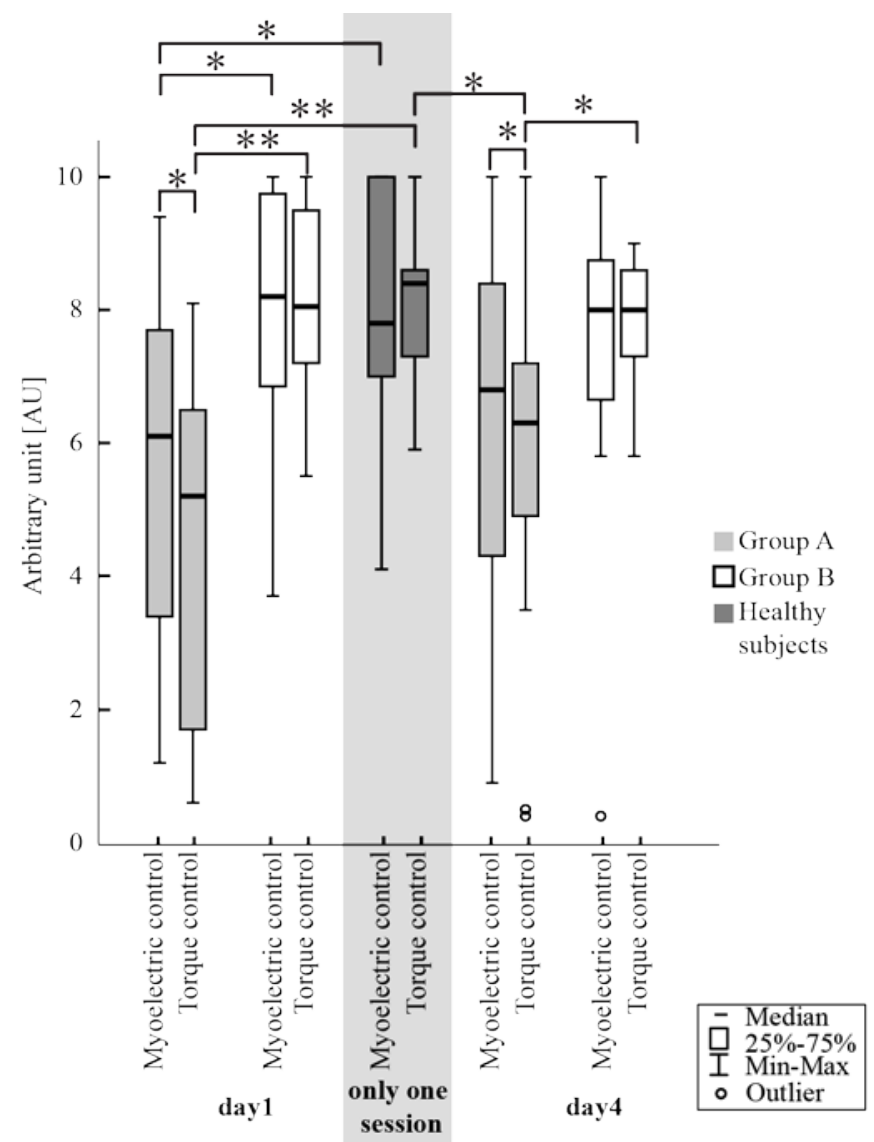

Figure 15: Subjective opinion for group A (light gray boxplots), group B (white boxplots) and healthy subjects (dark grey boxplots) about the ease of use of each control modality after day 1 (left) and session 4(b). 
(Figure 15). The median responses about ease of system's use increased while the variability decreased at day 4 in comparison to day 1 (higher median, Q1 and Q3 values and narrower IQ-ranges). However, no significant differences were found.

According to the neurophysiological measurements, group A (light grey boxplots) and group B (white boxplots) and all patients together (dark grey boxplots) activated their muscles similarly using myoelectric (EMG-S) and torque control (TORQUE-S) with respect to the healthy subjects (no significant differences were found), Figure 16. This implies that stroke patients can recruit their muscles with myoelectric control (simple thresholding strategy) as they do it when applying residual force with torque control. Finally, group B activated their muscles using torque $(p<0.01)$ and myoelectric control $(p<0.001)$ more similarly to the healthy volunteers than group A did, as expected.

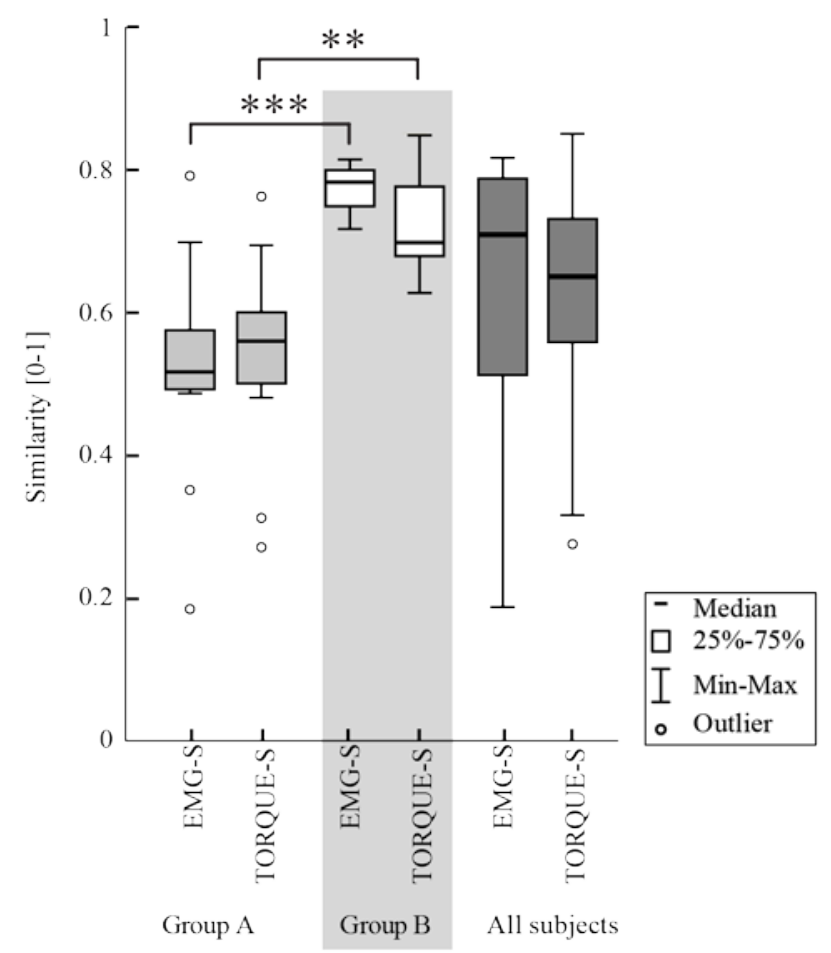

Figure 16: Results of the muscle activation similarity over all tasks for group A (light gray boxplots), group B (white boxplots) and all patients (dark grey boxplots). The muscle activation of all tasks was obtained using the NNMF algorithm (see section 2.3.3) from 16 surface EMG channels recorded session 4 for the patient groups and at the only daily session for the healthy volunteers (see section 2.2.2). 
The median reconstruction error of the muscles synergies for all tasks and directions was $77 \%$ for the healthy subjects group (third quartile Q3 $=81 \%$ and first quartile $\mathrm{Q} 1=70 \%)$ and $78 \%(\mathrm{Q} 3=84 \%$ and $\mathrm{Q} 1=72 \%)$ for the stroke patients.

The synergy set from the healthy subjects group can be visualized in Figure 17. Subjects recruited muscles very similarly during myoelectric and torque control (the

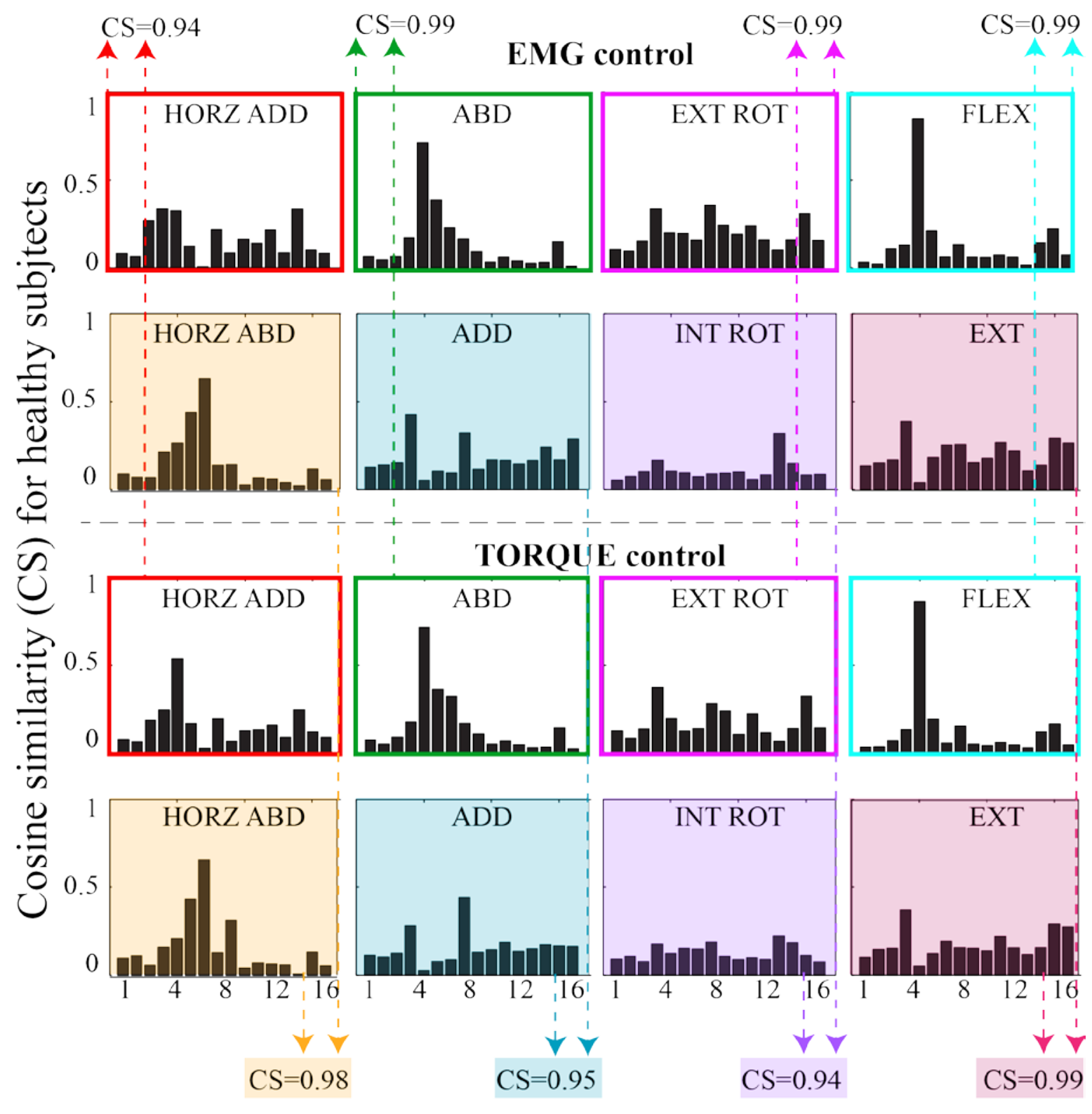

Overall median $=0.98$

Muscles

Figure 17: Median synergy set for the healthy subject group $(n=10)$. This is the reference of muscle recruitment for using the robot with myoelectric (upper two rows) and torque control (lower two rows). Healthy subjects recruited muscle patterns very similarly when using myoelectric control and torque control (overall median similarity $=0.98$ of all tasks and control modalities). 
overall median of all tasks was 0.98 ). This indicates a high reliability of synergy extraction across tasks and control modalities for the healthy subjects groups. Therefore, the reference of muscle activations from the healthy subjects is a reliable reference for driving conclusions of the muscle recruitment patterns of patients and assessing the EMG-S and TORQUE-S of patients.

In Figure 18, the synergy sets for a representative subject from group B and the

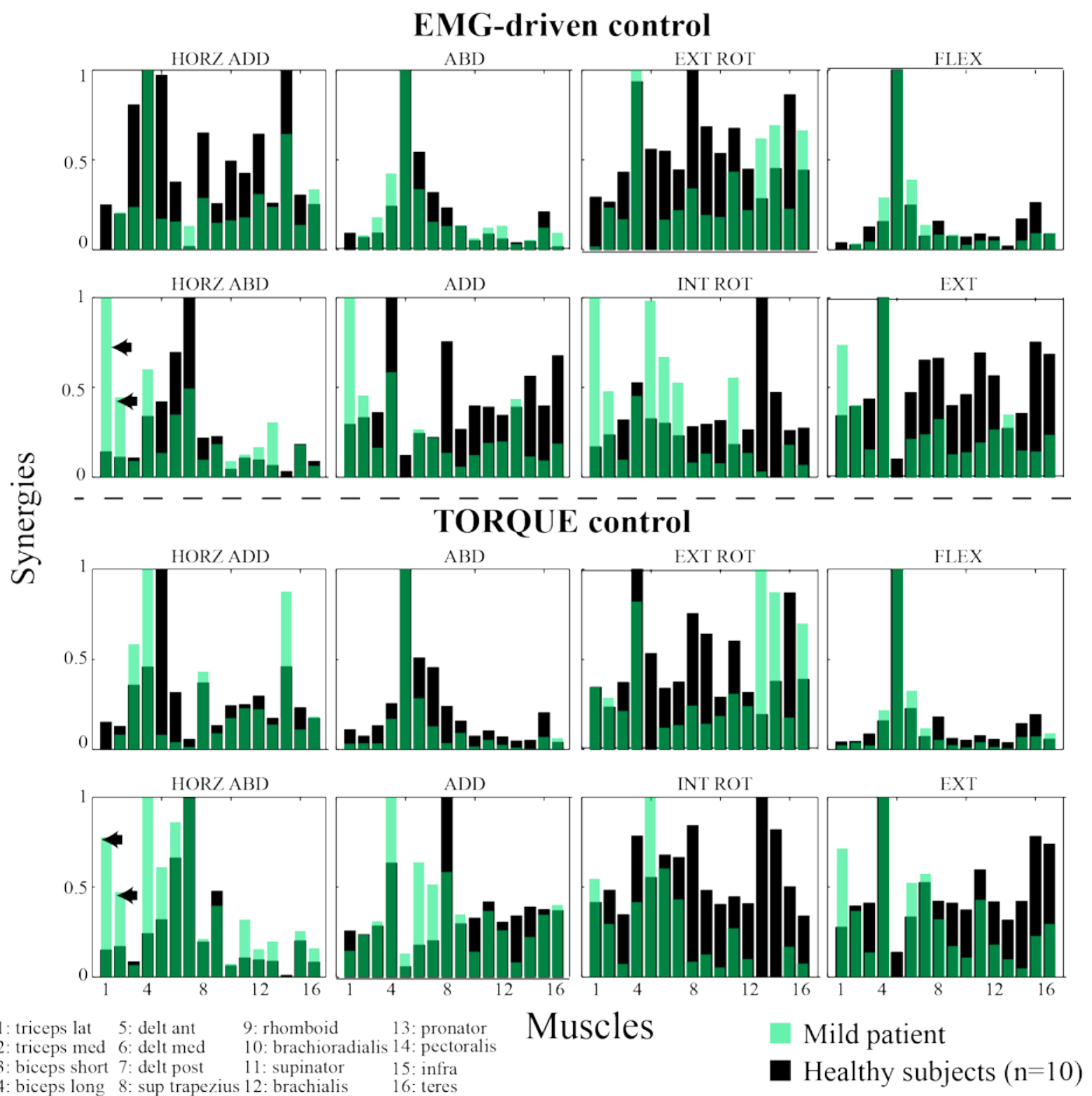

Figure 18: Synergies for a representative patient from group B (green bars) and the median synergies of healthy subjects (black bars) for all single-DoF tasks and both control modalities EMG-driven control (upper two rows) and torque control (lower two rows). The EMG-S for this subject was 0.8 and the TORQUE-S for this subject was 0.85 . The triceps brachii, lateral and medial head $(\leftarrow)$, was markedly more activated in the HORZ ABD task in comparison to the median activation of the same muscle for the healthy subjects. 
synergy set from the healthy subjects group ( $n=10$, reference muscle recruitment) for myoelectric and torque control can be visualized. The EMG-S and TORQUE-S for this representative patient were 0.8 and 0.85 , respectively. The recruitment patterns for the tasks ABD/ADD and FE (flexion/extension) were more similar to the median muscle activation of the healthy subjects group ( 0.83 and 0.89 for myoelectric control and 0.89 and 0.91 for torque control, respectively) in comparison to the tasks HORZ ADD/ABD and I/E (0.76 and 0.71 for myoelectric control and 0.77 and 0.68 for torque control, respectively). For the task HORZ ABD, the triceps brachii, lateral and medial head $(\leftarrow)$, of the mildly impaired patient was markedly more activated than for the healthy subjects group. This muscle was probably more recruited due to compensatory strategies developed by the patient after the lesion.

In contrast, the synergy sets for a representative subject from group $A$ and the synergy set from the healthy subjects group ( $n=10$, reference muscle activations) for myoelectric and torque control are shown in Figure 19. The similarity between a representative patient from group $A$ and the healthy subjects group (reference muscle activations) was lower. The EMG-S and TORQUE-S for this representative patient were 0.7 and 0.69 , respectively. For this patient with stronger muscle weakness, the attempt of voluntary movements resulted in activation of the abnormal "flexor synergies". The presence of unwanted motor synergies after stroke has been described in the literature for over 30 years [68]. The severely impaired patient activated the biceps brachii, long head $(\nabla)$, markedly stronger in comparison to the healthy subjects group in the tasks HORZ ABD, ABD, I and $F$ during myoelectric control and HORZ ADD/ABD. ABD/ADD and F during torque control. For the tasks ABD/ADD and FE during torque and myoelectric control, the biceps brachii, short 
EMG-driven control
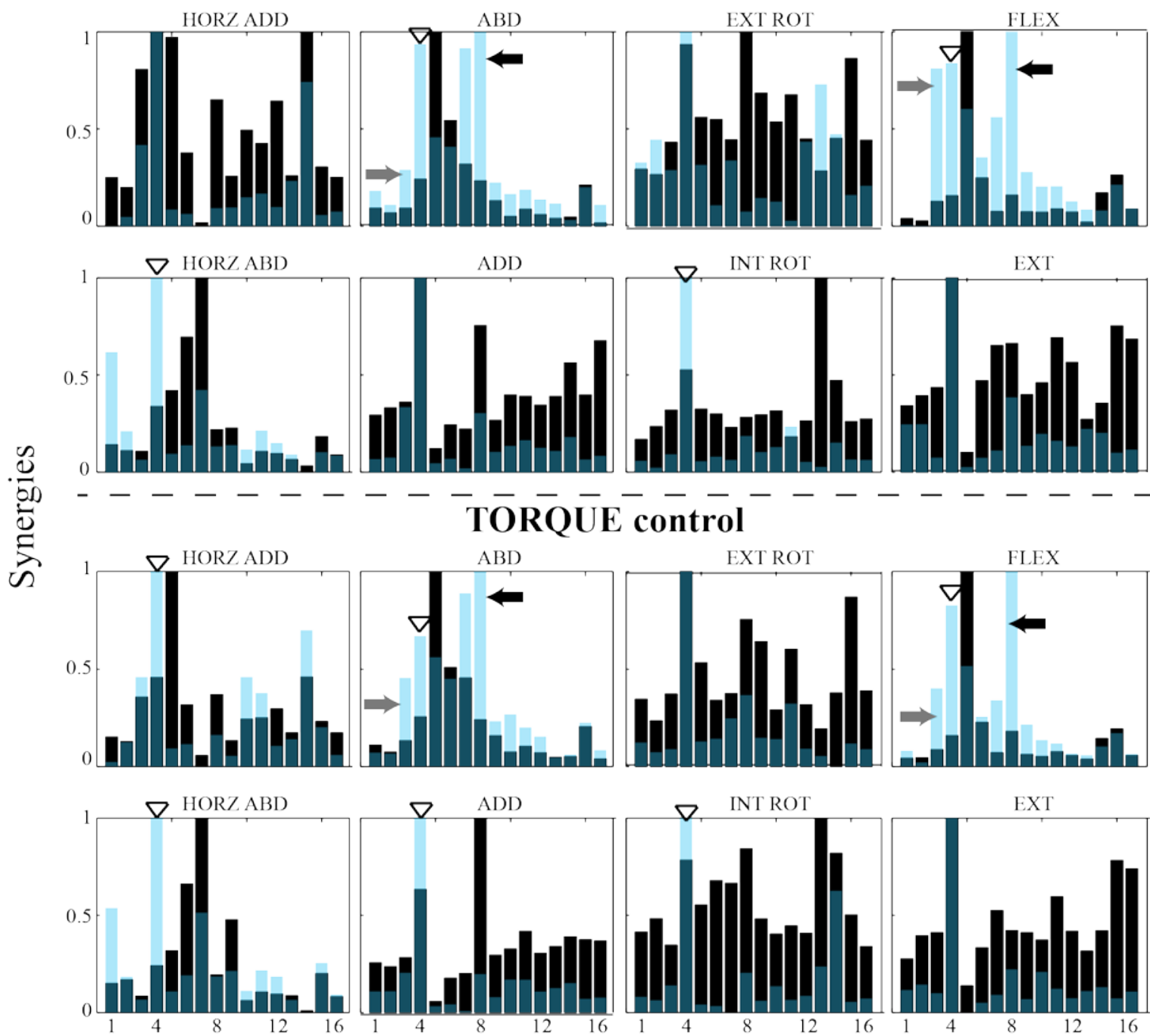

9: rhomboid

13: pronator

10: brachioradialis 14: pectoralis

Muscles

$\nabla \quad$ INT ROT

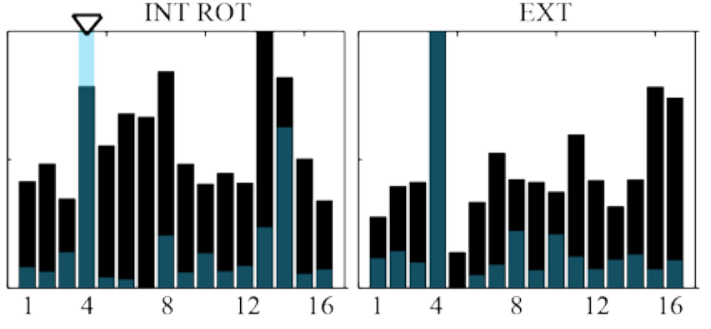

2: triceps med 6: delt med

11: supinator

15: infra

Severe patient

Healthy subjects $(n=10)$

Figure 19: Synergies for a representative patient from group A (light blue bars) and the median synergies of the healthy subjects group (black bars) for all single-DoF tasks and both control modalities EMG-driven control (upper two rows) and torque control (lower two rows). The EMG-S for this subject was 0.7 and the TORQUE-S for this subject was 0.69 . The biceps brachii long head $(\nabla)$, was markedly more activated in five out of eight tasks of myoelectric control and in sex out of eight tasks of torque control in comparison to the median activation of the same muscle for the healthy subjects. For the tasks ABDIADD and FLEX/EXT in torque and myoelectric control, the biceps brachii short head $(\rightarrow)$ and the superior trapezius $(\leftarrow)$ were markedly more activated in comparison to the median activation of the same muscle for the healthy subjects.

head $(\rightarrow)$, was also strongly activated and the patient also compensated with the

superior trapezius $(\leftarrow)$.

\subsubsection{Discussion}

This engineering study showed that the RehaARM with both control modalities

permitted patients, even those severely affected, to understand how execute a task 
of the protocol proposed in this study. The control of the robot for performing singleDoF movements with muscles signals (myoelectric control) and residual force (torque control) made patients aware of the actions they have to take in order to start and continuously complete a task. For reaching this goal, the protocol included simpler, single-DoF shoulder tasks that are relevant for ADLs.

The robot assisted the patients along those tasks, thereby limiting excursions along other DoFs (maximum torque setting). This facilitation contributed to the patients' understanding and awareness of their motor control to perform a task with myoelectric and torque control. The elbow and the hand joint were fixed and this also facilitated the shoulder tasks. Even severely impaired patients were able to drive the robot into a movement (more easily with myoelectric than with torque control) while mild patients were able to actively perform brighter shoulder movements (with myoelectric and torque control). Therefore, the RehaARM system can be used fortask specific training according to individual needs.

The mild and severe-to-moderate patients were able to use both control modalities and performed better over the four consecutive days. The mild group had higher median and Q1 values over consecutive days with both control modalities. The severe-to-moderate group performed markedly better over consecutive days and significantly better at day 4 in comparison to day 1 with both control modalities $(p<0.05)$. Physiotherapists could adjust the level of difficulty of the training to the patient's ability with both modalities to avoid compensation as much as possible. Therefore, the monotonic increase in the movement completion rate over multiple sessions reflects improvement in motor control.

The mild patients performed similarly well (median CR $\sim 40$ over all days) with both control modalities to the healthy subjects group who all executed 40 task repetitions 
in $20 \mathrm{~min}$. The severe-to-moderate impairment group executed 18.5 and 15 (median) task repetitions over all days with myoelectric and torque control, respectively. That is approximately $46 \%$ and $38 \%$ of the performance of the healthy subjects (mild patients). This indicates that group A had more difficulty using torque control while group B used both modalities equally similar. In addition, according to the questionnaire, group A thought they were able to use myoelectric control more easily than torque control while group B and healthy subjects thought both modalities were similarly ease to use.

Finally, stroke patients had similar synergistic motor control with modalities, myoelectric control and torque control. Even though, for myoelectric control (simple thresholding) just one triggering muscle was used per task, the synergistic activation of muscle groups was possible for executing the task. This indicates that for taskspecific training, the most important muscle can be chosen as triggering muscle for a certain task. This is a very important aspect for the usability of the presented myoelectric control algorithm for upper limb rehabilitation robots.

\subsection{Study 3: Myoelectric Robot-Assisted Rehabilitation for the Upper Limb after Stroke}

\subsubsection{Abstract}

Objective. Robot-assisted therapy is a promising treatment providing high-dosage exercise therapy for improving motor function after stroke and providing likely benefits to generic ADLs. The novel EMG-driven RehaARM for the shoulder (active joint), its therapeutic concept and the results of a pilot clinical study conducted using this system are presented here. Approach. In this pilot study, 20 patients were eligible and each underwent 2 hours of daily therapy delivered 5 days per week, for 17 days ( -3.5 weeks). The 2 hours of therapy consisted of 1 hour of RehaARM robotic-assisted therapy and 1 hour of conventional therapy. The primary outcome 
measures were the Fugl-Meyer Upper Extremity (FMA-UE) and Functional Independence Measure (FIM) scales. In an explorative part of this study, we used a historical comparison group $(n=19)$ from a prior clinical trial which had conventional therapy for 20 days ( 4 weeks), also for 2 hours daily. We compared the effectiveness of the treatment for the RehaARM group with that of the conventional therapy group. The sets of patients were comparable at baseline (age, sex, impairment level, etc.). The objective was to compare the treatments in terms of clinical effect. Main results. The treatment with the RehaARM significantly improved motor control (FMA-UE) and activity (FIM) scores. There was a statistically significant increase in FMA-UE and FIM scores following participation in the training program $(p<0.001)$ with large effect sizes ( $r=0.54$ and 0.55 , respectively). The median score of the FMA-UE increased for pre- to post-treatment (from 12.5 to 16.5 ) and the FIM also increased (from 98 to 109). The scores for the conventional therapy also increased for pre- to posttreatment (from 16 to 20 on the FMA-UE scale) and (from 97 to 107 on the FIM scale). There was no significant difference between the RehaARM and conventional therapy groups in the pre- and post-treatment increments, measured on the FMA-UE and FIM scales. Based on the FMA-UE scores, the sample sizes needed to determine the effectiveness of the treatment were similar for conventional therapy (n $=27, \mathrm{p}<0.05$; power $=0.8)$ and RehaARM therapy $(\mathrm{n}=28, \mathrm{p}<0.05$; power $=0.8)$. There were no occurrences of serious adverse events related to the study. Significance. EMG-controlled robotic therapy for upper limb after stroke was carried out successfully with severely, moderately and mildly impaired hemiparetic stroke survivors. The results of this study indicate that training with an arm robot is safe and improves both body functions and independence in ADLs equally well as 
conventional therapy alone at a comparable dose and dosage. This technique is promising as a new modality for active-assistive training after stroke.

\subsubsection{Methods}

\subsubsection{Phase II Clinical Study with the RehaARM}

This study is a phase II clinical trial - also known as pilot trial in clinical research since it evaluated the efficacy and safety of the therapeutic treatment for the recovery of the upper limb after stroke with the RehaARM system. This study did neither have a control group nor a randomization. It should be used to optimize the design of a subsequent randomized phase III clinical trial - also known as pivotal trial - with one or more control groups and a larger sample size per group in order to compare the efficacy of robotic-assisted therapy plus conventional therapy with other treatments. For example, this study can guide the effective use of limited (financial and nonfinancial) resources essential for a successfully performed phase III trials [69].

The cohort of post-stroke patients considered for the study was selected from admissions to the Cerebrovascular Disease Unit of the Fondazione Ospedale San Camillo. Within this cohort of patients, those suffering from hemiparesis due to a first stroke in the region of the middle cerebral artery (MCA), anterior cerebral artery (ACA) and posterior cerebral artery (PCA) were screened for this study. CT/MRI scan indicated various combinations of brain lesions, i.e. large damage involving most of the vascular territory of the MCA, PCA or ACA or more discrete lesions of the cortical and/or subcortical areas supplied by branches of these arteries. This study included hemorrhagic and ischemic (cerebral infarction) stroke patients and one case of subarachnoid hemorrhage. Moreover, the patients included in the study had a Motor Arm sub-score between 1 and 3 on the Italian version of the National 
Institutes of Health Stroke Scale (It-NIHSS) [70]. The inclusion and exclusion criteria were the same as for study 1 and 2 (see section 3.1.2.2). In addition, the patients were further divided in subgroups based on: (1) the baseline severity of motor impairment detected with the FMA-UE scale, similarly as for study 1 and 2 (group A is severe-to-moderate and group B is mild; see section 3.1.2.2); (2) the duration of stroke to rehabilitation interval, SRI (between 1 and 3 months, between 4 and 12 months, exceeding 12 months); and (3) type of stroke and cerebral infarction. The types of stroke were ischemic stroke, intracerebral hemorrhage (ICH) and subarachnoid hemorrhage (SAH). The types of cerebral infarction were classified according to the Oxford Community Stroke Project classification (OCSP, also known as the Bamford or Oxford classification) using $\mathrm{CT}^{7}$ and $\mathrm{MRI}^{8}$. This classification groups types of cerebral infarction primarily based on clinical symptoms. In this study, there were patients with total anterior cerebral infarct (TACI), partial anterior circulation infarct $(\mathrm{PACl})$ and posterior circulation infarct $(\mathrm{POCl})$. There were no lacunar infarct (LACl) patients. LACl patients present a pure motor stroke, pure sensory stroke or ataxic hemiparesis.

$\mathrm{TACl}$ refers to when a large cortical stroke occurred in both the deep and superficial areas of the MCA/ACA. TACI is diagnosed based on the following three symptoms: (1) homonymous hemianopia; (2) ipsilateral motor and sensory deficits, in which at least two out of three areas of the face, arms and legs are affected; (3) higher cerebral dysfunction (e.g. dysphasia, visuospatial disorder, decreased levels of consciousness). The volume of the infarct in patients with the complete clinical

\footnotetext{
${ }^{7}$ Computer tomography

${ }^{8}$ Magnetic resonance imaging
} 
syndrome is significantly greater than that in patients with more restricted deficits (i.e., LACI or PACI) [71].

The PACl group consists of cerebral infarcts affecting more restricted areas, relative to $\mathrm{TACl}$, of the MCA/ACA area. They are caused by the occlusion of the upper division of the MCA in case there are no visual field deficits or of the lower division in case of minor motor/sensory deficits. Patients show two of the three syndromes of $\mathrm{TACl}$, higher cerebral dysfunction alone, or a motor/sensory deficit less severe than those classified as LACI (e.g. confined to one limb, or to face and hand but not to the whole arm) [71].

$\mathrm{POCl}$ is a group of cerebral infarction associated with the brainstem, cerebellum, and occipital lobes. The symptoms presented by patients in this group are the following: "ipsilateral cranial nerve palsy with contralateral motor and/or sensory deficit; bilateral motor and/or sensory deficit; disorder of conjugate eye movement; cerebellar dysfunction without ipsilateral long-tract deficit (i.e., ataxic hemiparesis); or isolated homonymous visual field defect" (page 1522 in [71]).

The TACl group has a negligible chance of good functional outcome and mortality is high and that $\mathrm{POCl}$ has the best chance of good functional outcome [71]. For the $\mathrm{PACl}$ group, no information regarding this improvement chance was found.

This study is registered with ClinicalTrials.gov, identifier: NCT02321254. Twenty out of the twenty four enrolled patients (characteristics in Table 9) completed the rehabilitation program. Most of the recruited patients were severe-to-moderate in a subacute phase and suffered a cerebral infarct (Table 9). The patients underwent two hours of daily therapy for 17 days (approx. 3.5 weeks). The two-hour daily therapy consisted of one hour of RehaARM robotic-assisted therapy and one hour of conventional therapy. The daily therapy was provided on continuous days when 


\begin{tabular}{|c|c|c|}
\hline Patients & & \\
\hline Sex (M/F) & & $12 / 8(60 \%, 40 \%)$ \\
\hline Age (years) & median(Q3;Q1) & $52.7(67.9 ; 44.4)$ \\
\hline \multirow[t]{2}{*}{ Upper limb motor impairment } & Severe-to-moderate, group A (FMA-UE $\leq 38$ ) & $14(70 \%)$ \\
\hline & Mild, group B (FMA-UE>38) & $6(30 \%)$ \\
\hline \multirow[t]{4}{*}{ SRI (months) } & median(Q3;Q1) & $5.5(8.6 ; 3.1)$ \\
\hline & $\leq 3$ months & $4(20 \%)$ \\
\hline & $3<$ months $<12$ & $13(65 \%)$ \\
\hline & $\geq 12$ months & $3(15 \%)$ \\
\hline \multirow[t]{3}{*}{ Stroke classification } & Ischemic & $13(65 \%)$ \\
\hline & $\mathrm{ICH}$ & $6(30 \%)$ \\
\hline & $\mathrm{SAH}$ & $1(5 \%)$ \\
\hline
\end{tabular}

possible. In case of missed sessions, they were rescheduled the next day, in order to complete the full assigned rehabilitation program of 17 days of therapy. Up to three continuously missed sessions could be rescheduled. The minimum time for each session of conventional or robotic therapy (excluding time for preparation, diagnostics, and documentation) was 50 min. Patient preparation for the robotic therapy took approx. 5-10 min. Four subjects withdrew from the study because of scheduling conflicts.

The conventional therapy was delivered as in regular rehabilitation, as was done for the historical conventional therapy group (see section 3.3.2.2). The RehaARM therapy involved performing single-DoF shoulder tasks (same tasks as in study 1 and 2, section 3.1.2.2) for a period of one hour with the robot's assistance. The therapist was present at every session for the entire duration, as in a standard oneto-one setting. The therapist's role was to manage the robot interface to adapt it to the current patient's physical condition and to guide the patient with verbal instructions in case difficulties arose during the execution of the exercises.

The Fugl-Meyer upper extremity (FMA-UE) and the Functional Independence Measure (FIM) scales were chosen as outcome measures for the upper limb motor 
function and the independence in ADLs, respectively. In addition to the primary outcomes, secondary outcome measurements were taken on a set of clinical scales and kinematic and novel neurophysiological scales (see section 2.3).

The interventions were carried out by two therapists and baseline and post-treatment assessments were carried out by a different therapist. The baseline assessment was carried out before the start of the treatment (session 1) and the post-treatment assessment was carried out after session 17. The baseline and post-treatment assessments included all clinical assessments (see section 2.3.1) and the kinematic measures (see section 2.3.2). The EMG measurements of 16 upper limb muscles were carried out at session one and session 17 in order to obtain the neurophysiological assessment at baseline and post-treatment (see section 2.3.3).

\subsubsection{Comparison of the RehaARM therapy with a Historical Conventional} Therapy (CVT) group

The historical CVT group $(n=19)$ was obtained from the database of Fondazione Ospedale San Camillo. The dataset was constructed from 2003 to 2012 for research purposes. It includes patients who suffered from a first-ever supratentorial stroke (ischemic and/or hemorrhagic) in the right and left hemisphere. The baseline characteristics of these patients (sex, age, impairment level, etc.) were comparable to the baseline characteristics of the RehaARM group (see section 3.3.4.2). The patients in this comparison group underwent conventional therapy for 20 sessions (4 weeks), two hours daily. The interventions were planed continuously if possible and missed sessions were rescheduled to the next day.

The conventional therapy program had common rehabilitation treatment given to patients after stroke in inpatient care. The techniques for restoring upper limb and lower limb motor functions were based on the Bobath principles [40], [72]. The upper-limb therapy included mobilization, motor tasks of increasing difficulty without 
and with postural control depending on residual motor function, games, activities of daily living, or any combination of the four. The amount of upper-limb and lower limb therapy was tailored to the patient's needs and goals.

A subgroup of 19 out of 20 subjects from the RehaARM therapy group were selected in order to assess whether the treatment with the RehaARM reduces motor impairment and improves ability more effectively than conventional therapy does. The subject from the RehaARM therapy group with SAH was excluded since the conventional therapy group did not include SAH patients.

In this study, the true difference in effectiveness between the RehaARM roboticassisted therapy and conventional therapy cannot be deduced. Our comparison is solely explorative. By using historical comparison groups in clinical trials differences in concomitant factors could bias outcomes. However, we strived to minimize phenotype variability and baseline factors (age, sex, impairment level, etc.) were comparable between the groups. Therefore, we believe we have sufficiently reduced potential biases to draw reliable conclusions.

\subsubsection{Data Analysis}

Differences in demographic and clinical characteristics between the CVT and the RehaARM groups at baseline were analyzed using the $x 2$ test, whereas median age differences were analyzed with a Wilcoxon matched-pairs test. Wilcoxon and MannWhitney $U$ tests were used to study the outcome differences within and between CVT and RehaARM treatment groups, respectively. The effect sizes were calculated based on the Wilcoxon matched-pairs test. Effect sizes $\geq 0.5$ are considered strong, $0.3 \leq$ effect sizes $<0.5$ are considered medium and $0.1 \leq$ effect sizes $<0.3$ are considered small [73], [74]. 
The required sample sizes for the CVT and the RehaARM group was calculated as described by Rosner [75] and using the software G*Power Statistical Power Analyses for Windows ${ }^{9}$ assuming a normal parent distribution and using a two-sided Wilcoxon matched-pairs test. The alpha level ( $p$-value) was set to 0.05 and the beta level was set to 0.2 in order to have a statistical power of $80 \%$ for the sample calculation.

As for study 1 and 2, the recorded EMG signals were manually inspected to remove the task repetitions with evident artifacts due to electrode displacements or accidental contacts with the robotic parts. The maximum values of the activation profiles for the triggering and scapular muscles $\left(\max \left(A_{E M G}^{k}\right)\right)$ for each task and subject group were calculated as the median of the maximum activation values for each trial as in study 1 (see also section 3.1.3.2). The correlations between the neurophysiological and clinical measures were calculated using the Pearson's correlation.

As for the previous studies, nonparametric tests were used because the data distributions were not normal and the number of subjects was small for parametric tests, as determined by the Shapiro-Wilk Test. The statistical differences, normality rejections and correlations were tested at a significance level of $p<0.05$. The data and statistical differences are indicated in the text, boxplots and tables as for the previous studies (see also section 3.1.3.2). The statistical tests were done in the software IBM SPSS Statistics 22.

\footnotetext{
${ }^{9}$ http://www.gpower.hhu.de/ from the Düsseldorf University
} 


\subsubsection{Results}

\subsubsection{Phase II Clinical Study with the RehaARM}

The results for all patients indicated that motor function significantly improved after treatment. There was a significant increase $(p<0.001)$ of the FMA-UE score with a large effect size $(r=0.54)$. The median score increased for pre- to post-treatment (from 12.5 to 16.5). See Table 10, overall results.

The motor function significantly improved for group A $(p<0.01)$ and group $B(p<0.05)$ after therapy with a large effect size $(r=0.53$ and 0.59 , respectively). The median score on the FMA-UE increased pre- to post-treatment (from 9.5 to 12) for group A and from 50.5 to 60 for group B. See Table 10, motor impairment.

Subacute patients showed a significant improvement $(p<0.01)$ after treatment with a large effect size $(r=0.52)$. The median score on the FMA-UE increased pre- to post-treatment (from 11 to 17). There was no significant increase for the acute and chronic group because the sample size was too small, resulting in a low statistical power. The median score on the FMA-UE increased pre- to post-treatment both for the acute group (from 31.5 to 36.5 ) and for the chronic group (from 12 to 16). See

\begin{tabular}{|c|c|c|c|c|c|c|}
\hline & & $\mathbf{N}$ & $\begin{array}{l}\text { PRE-Treatment } \\
\text { Median(Q3;Q1) }\end{array}$ & $\begin{array}{l}\text { POST-Treatment } \\
\text { Median(Q3;Q1) }\end{array}$ & $\begin{array}{c}\text { Effect size } \\
r\end{array}$ & $\begin{array}{l}\text { Nover } \\
\text { MCID }\end{array}$ \\
\hline Overall & & 20 & $12.5(41.3 ; 8.3)$ & $16.5(45.5 ; 10.3)^{* * *}$ & 0.54 & $7(35 \%)$ \\
\hline \multirow[t]{2}{*}{ Motor impairment } & Group A & 14 & $9.5(13.8 ; 7.8)$ & $12(17.5 ; 9)^{* *}$ & 0.53 & $3(21 \%)$ \\
\hline & Group B & 6 & $50.5(55.8 ; 41.3)$ & $60(62 ; 43.5)^{*}$ & 0.59 & $4(67 \%)$ \\
\hline \multirow{3}{*}{$\begin{array}{l}\text { Stroke to Rehabilitation Interval } \\
\text { (SRI) }\end{array}$} & $\leq 3$ months & 4 & $31.5(56 ; 9.3)$ & $36.5(61.5 ; 13)$ & I & $2(50 \%)$ \\
\hline & $3<$ months $<12$ & 13 & $11(40.5 ; 7.5)$ & $17(45 ; 9)^{* *}$ & 0.52 & $5(38 \%)$ \\
\hline & $\geq 12$ months & 3 & $12(16 ; 9)$ & $16(19 ; 11)$ & l & 0 \\
\hline \multirow[t]{3}{*}{ Stroke classification Ischemic } & TACI & 5 & $12(27.5 ; 6.5)$ & $13(29 ; 8)$ & / & 0 \\
\hline & PACI & 6 & $13(52 ; 8.8)$ & $16(60.5 ; 10.5)^{*}$ & 0.58 & $2(33 \%)$ \\
\hline & POCI & 2 & $24(27.8 ; 8.3)$ & $31.5(34.5 ; 12.8)$ & I & $2(100 \%)$ \\
\hline \multirow[t]{2}{*}{ Hemorrhagic } & $\mathrm{ICH}$ & 6 & $17(52 ; 7)$ & $18(60.5 ; 8.8)^{*}$ & 0.58 & $2(33 \%)$ \\
\hline & $\mathrm{SAH}$ & 1 & 39 & 44 & / & $1(100 \%)$ \\
\hline
\end{tabular}


Table 10, SRI.

The PACl group showed a significant improvement $(p<0.05)$ after treatment with a large effect size $(r=0.58)$. The median score on the FMA-UE increased for pre- to post-treatment (from 13 to 16). The median FMA-UE score did not significantly change for the TACI group. For the POCI group, due to the small sample size $(n=2)$, and subsequently low statistical power, no statistics are reported. The median score on the FMA-UE increased pre- to post-treatment for the TACl group (from 12 to 13) and for the POCI group (from 24 to 31). See Table 10, ischemic stroke.

The ICH group showed a significant improvement $(p<0.05)$ after treatment with a large effect size $(r=0.58)$. The median score on the FMA-UE increased pre- to posttreatment (from 17 to 18). For the patient with the SAH, the score increased by five points (from 39 to 44). See Table 10, hemorrhagic stroke.

There were 7 out of the 20 patients (35\%) that gained at least 5 points on the FMAUE scale. In group A 3 of the 14 patients (21\%) and in group B 4 of the $6(67 \%)$ patients also achieved at least 5 points. About a third of the patients with ischemic (4/13) and $\mathrm{ICH}(2 / 6)$ also achieved at least five points.

\begin{tabular}{|c|c|c|c|c|c|c|}
\hline & & $\mathbf{N}$ & $\begin{array}{l}\text { PRE-Treatment } \\
\text { Median(Q3;Q1) }\end{array}$ & $\begin{array}{l}\text { POST-Treatment } \\
\text { Median(Q3;Q1) }\end{array}$ & Effect size & $\begin{array}{l}\text { N over } \\
\text { MCID }\end{array}$ \\
\hline Overall & & 20 & $98(110 ; 81.3)$ & $109(117.8 ; 94)^{* * *}$ & 0.55 & $3(15 \%)$ \\
\hline \multirow[t]{2}{*}{ Motor impairment } & Group A & 14 & 98.5(108.5;81) & $104(115.3 ; 91.3)^{* *}$ & 0.58 & $1(1 \%)$ \\
\hline & Group B & 6 & $95.5(112.5 ; 78)$ & 113.5(121.3;97.5) & I & $2(33 \%)$ \\
\hline \multirow[t]{3}{*}{ Stroke to Rehabilitation Interval } & $\leq 3$ months & 4 & $87(97.3 ; 70)$ & $97.5(116.8 ; 86.5)$ & l & $1(25 \%)$ \\
\hline & $3<$ months $<12$ & 13 & $98(108 ; 81)$ & $107(116.5 ; 88.5)^{* *}$ & 0.55 & $2(15 \%)$ \\
\hline & $\geq 12$ months & 3 & $113(119 ; 107)$ & $114(119 ; 111)$ & I & 0 \\
\hline \multirow[t]{3}{*}{ Stroke classification } & TACI & 5 & $99(109 ; 67)$ & 101(112;83)* & 0.65 & $1(20 \%)$ \\
\hline & PACI & 6 & $94(114 ; 78)$ & $105(120.5 ; 91.5)^{*}$ & 0.58 & $1(17 \%)$ \\
\hline & POCI & 2 & $93(78.8 ; 60.8)$ & 106.5(89.3;70.5) & I & 0 \\
\hline \multirow[t]{2}{*}{ Hemorrhagic } & ICH & 6 & 101(117.5;87.8) & 112.5(119.5;99.5) & / & $1(17 \%)$ \\
\hline & SAH & 1 & 98 & 102 & 1 & 0 \\
\hline
\end{tabular}




\begin{tabular}{|c|c|c|c|}
\hline OUTCOME & $\begin{array}{l}\text { PRE-Treatment } \\
\text { Median(Q3;Q1) }\end{array}$ & $\begin{array}{l}\text { POST-Treatment } \\
\text { Median(Q3;Q1) }\end{array}$ & $\begin{array}{c}\text { Effect size } \\
r\end{array}$ \\
\hline Motor FIM (77) & $55.5(66.8 ; 40.8)$ & $63.5(71.5 ; 48.5)^{* *}$ & 0.49 \\
\hline FM (152) & $90(108.5 ; 77.5)$ & $91(124.8 ; 86)^{* * *}$ & 0.54 \\
\hline MAS (20) & $1(4.8 ; 0)$ & $2(4 ; 0)$ & l \\
\hline NHPT (9) & $0(0 ; 0)$ & $0(1 ; 0)$ & I \\
\hline RPS (36) & $6(27 ; 0.3)$ & $11(29.3 ; 3)^{* *}$ & 0.44 \\
\hline Shoulder_ABD $\left({ }^{\circ}\right)$ & $18(52.5 ; 6)$ & $37(69.3 ; 13)^{* * *}$ & 0.53 \\
\hline Shoulder_FLEX $\left(^{\circ}\right)$ & $24(73.5 ; 5.5)$ & $28.5(98 ; 12)^{*}$ & 0.37 \\
\hline Elbow_FLEX $\left({ }^{\circ}\right)$ & $69(141 ; 15)$ & $97(158 ; 20)^{*}$ & 0.4 \\
\hline
\end{tabular}

The results for all patients showed that the score in the activity scale (FIM) significantly improved after the therapy program. There was a significant increase $(p<0.001)$ with a large effect size $(r=0.55)$. The median score increased for pre- to post-treatment (from 98 to 109). See Table 11, overall results.

The severe group A showed a significant increase $(p<0.01)$ after treatment with a large effect size $(r=0.58)$. The median score increased for pre- to post-treatment (from 98.5 to 104). For group B, the median showed a non-significant increase (from 95.5 to 113.5). See Table 11, motor impairment.

The TACl and PACl group showed a significant increase $(p<0.05)$ with large effect sizes ( $r=0.65$ and 0.58 , respectively). The median score increased pre- to posttreatment for the TACl group (from 99 to 101) and for the PACl group (from 94 to 105; See Table X, stroke classification). For the POCI group due to the small sample size $(n=2)$ and low statistical power, no statistics are reported. The median score increased from 93 to 106.5. See Table 11, ischemic stroke.

The FIM scores showed a non-significant improvement for the $\mathrm{ICH}$ group and the SAH patient after treatment. The median scores increased pre- to post-treatment for 
Table 13 effect of therapy on the neurophysiological measurements

\begin{tabular}{|c|c|c|c|c|c|c|}
\hline & & & $\mathbf{N}$ & $\begin{array}{l}\text { PRE-Treatment } \\
\text { Median(Q3;Q1) }\end{array}$ & $\begin{array}{l}\text { POST-Treatment } \\
\text { Median(Q3;Q1) }\end{array}$ & $\begin{array}{c}\text { Effect size } \\
r\end{array}$ \\
\hline \multirow[t]{3}{*}{ EMG-S } & Overall & & 20 & $0.62(0.75 ; 0.51)$ & $0.69(0.8 ; 0.61)^{* * * *}$ & 0.62 \\
\hline & Motor impairment & Group A & 14 & $0.54(0.66 ; 0.5)$ & $0.63(0.75 ; 0.57)^{* * *}$ & 0.52 \\
\hline & & Group B & 6 & $0.74(0.8 ; 0.72)$ & $0.80(0.86 ; 0.77)^{*}$ & 0.35 \\
\hline \multirow[t]{3}{*}{ TORQUE-S } & Overall & & 20 & $0.62(0.72 ; 0.54)$ & $0.68(0.76 ; 0.57)$ & 0.31 \\
\hline & Motor impairment & Group A & 14 & $0.57(0.66 ; 0.51)$ & $0.61(0.72 ; 0.55)$ & 0.29 \\
\hline & & Group B & 6 & $0.72(0.79 ; 0.68)$ & $0.76(0.79 ; 0.70)$ & 0.12 \\
\hline
\end{tabular}

$\mathrm{ICH}$ (from 101 to 112.5) and for the SAH subject (from 98 to 102). See Table 11, Hemorrhagic stroke.

On the FIM scale 3 of the 20 patients (15\%) achieved gains of at least 22 points. In group A just 1 of the 14 patients (8\%) and in group B 2 of the 6 patients (33\%) achieved at least 22 points. For ischemic stroke, 2 of the 13 patients (15\%) and for $\mathrm{ICH} 1$ of the 6 patients (17\%) also achieved at least 22 points.

For the secondary measurements (Table 12), motor FIM, FM, RPS and all kinematic measures (shoulder_ABD, shoulder_FLEX and elbow_FLEX) showed significant improvement post-treatment $(p<0.05)$. Non-significant results were obtained on the activity scale NHPT for finger dexterity. This result is unsurprising considering the upper limb was treated proximally. Non-significant change was found in the impairment scale MAS. This indicates that there is no evidence that spasticity increased after the treatment which is a very positive result.

There was no significant difference between EMG-S and TORQUE-S at baseline. EMG-S significantly improved $(p<0.0001)$ with a large effect size $(r=0.62)$ after treatment. The median score increased pre- to post-treatment (from 0.62 to 0.69 ). Group A $(p<0.001)$ and group B $(p<0.05)$ showed a significant improvement after treatment with a large $(r=0.52)$ and medium effect $(r=0.35)$ size, respectively. The median score increased pre- to post-treatment for group A (from 0.54 to 0.63 ) and for group B (from 0.74 to 0.80). See Table 13. 

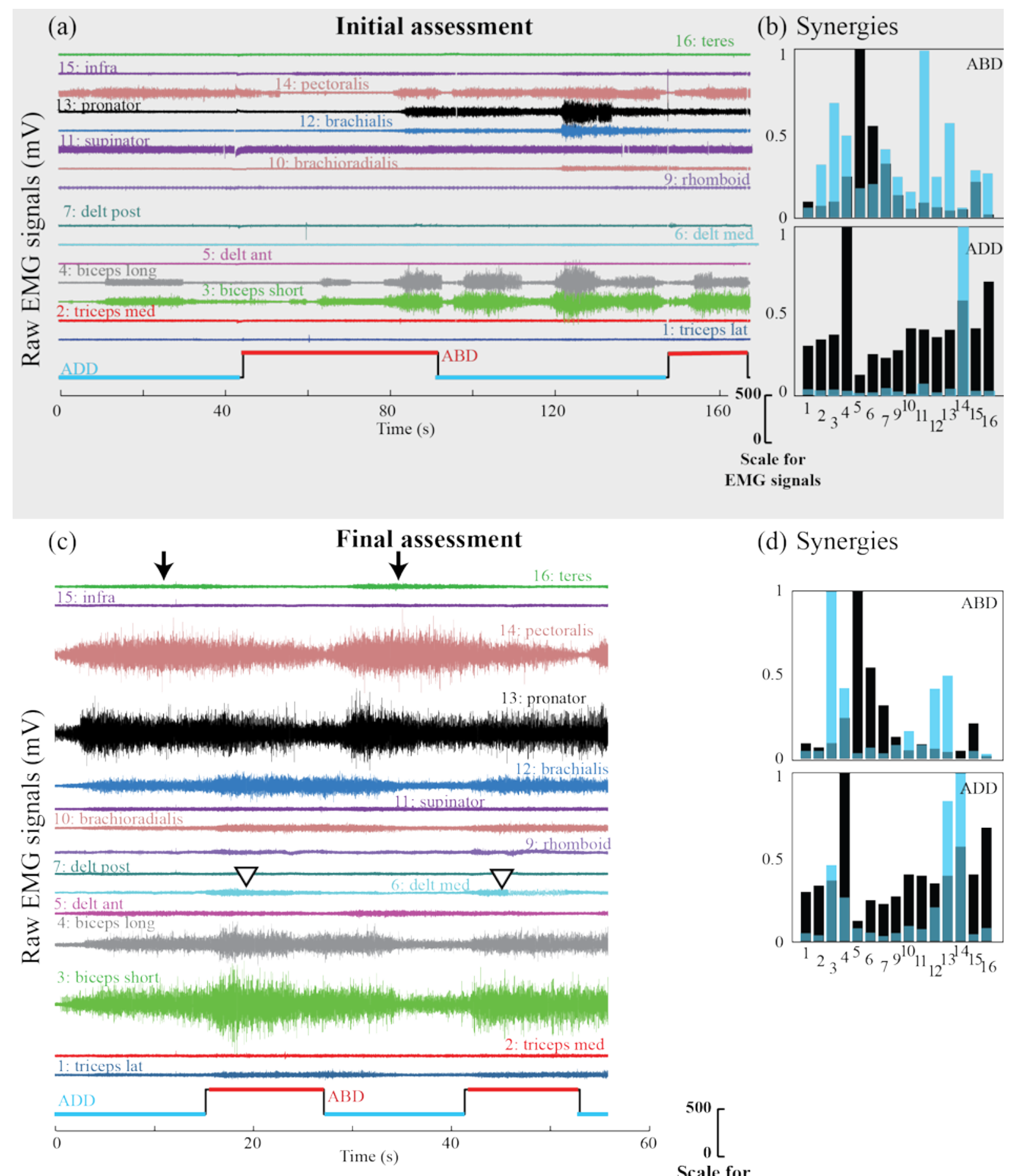

Figure 20: Raw EMG signals (a) and synergies (b) for the task ABDIADD in the initial assessment (upper grey-shaded area) of a representative patient from the severe-to-moderate group A and raw EMG signals (c) and synergies (d) for the same task in the final assessment (bottom white area) of the same patient. The signals from 15 muscles and the corresponding synergy modules are displayed. The muscle 8 (trapezius) was excluded because of strong artifacts. The raw EMG signals and the synergies show an improvement after treatment. In the final assessment, there was activity in the teres $(\downarrow)$ and medium deltoids $(\nabla)$ in contrast to the initial assessment. The black synergy modules in (b) and (d) represent the median muscle activity of the healthy subjects group and the light blue synergy modules represent the muscle activity of the patient. Note that the scale of the EMG signals in (a) and (c) is the same.

The results for all patients showed that TORQUE-S also improved after the therapy 
program. The median score increased non-significantly pre- to post-treatment (from 0.62 to 0.68$)$. The effect size of the treatment was medium $(r=0.31)$. TORQUE-S improved non-significantly for both groups after the therapy program. See Table 13. The neurophysiological measurements EMG-S and TORQUE-S significantly correlated $(p<0.01)$ with the FMA-UE scale in the initial $(0.73$ and 0.69 , respectively) and final assessment (0.83 and 0.69 , respectively). Contrastingly, no significant correlations were found between the neurophysiological scales and the FIM scale, Table 14.

The raw EMG signals for a representative subject from group A before and after the treatment are visualized in Figure 20. During the initial neurophysiological assessment (at day one, grey-shaded area in Figure 20), the patient could not execute the task ABD/ADD because the required muscles teres (for $A D D$ ) and medium deltoids (for $A B D$ ) were inactive. Nevertheless, the therapist allowed the patient to try to execute the task with guidance. As there was not activation, the therapist activated the passive mode and asked the patient to follow the movement with effort. During the final assessment (at day 17; white area in Figure 20), the patient was able to execute the task with complete autonomy and there was activation in the teres $(\downarrow)$ and the medium deltoids ( $\nabla)$ (Figure 20 (b)). The after treatment synergies in myoelectric control for the task ABD/ADD became present and more similar to the median synergies of the healthy subjects group. The median similarity for the task ABD/ADD improved pre- to post-treatment (from 0.26 to 0.43 ). However, the synergies were still markedly different from the synergies of the healthy subjects group.

The maximum activation of the rotator cuff muscles significantly improved for the severe-to-moderate group A after treatment. The maximum activation value of the 


\begin{tabular}{|c|c|c|}
\hline Assessment time & Variable & Pearson correlation \\
\hline \multirow[t]{4}{*}{ Initial } & EMG-S and FMA-UE & $0.734 * *$ \\
\hline & EMG-S and FIM & 0.079 \\
\hline & TORQUE-S and FMA-UE & $0.694 * *$ \\
\hline & TORQUE-S and FIM & -0.074 \\
\hline \multirow[t]{4}{*}{ final } & EMG-S and FMA-UE & $0.83^{* *}$ \\
\hline & EMG-S and FIM & 0.054 \\
\hline & TORQUE-S and FMA-UE & $0.693 * *$ \\
\hline & TORQUE-S and FIM & 0.122 \\
\hline
\end{tabular}

teres improved $(p<0.05)$ for the ADD and the EXT, similarly the maximum value of activation of the infraspinatus improved $(p<0.01)$ for the FLEX task.

No serious side effects occurred in this study. One patient from group B reported mild shoulder pain. Therefore, therapy was interrupted for three sessions and then resumed by narrowing the ROM without further adverse events. No further adverse events were reported.

\subsubsection{Comparison of the RehaARM therapy with a Historical Conventional Therapy group (CVT)}

At baseline (Table 15), the characteristics of the RehaARM therapy group and the CVT group were comparable on the variables: age, sex, SRI, stroke type and FMAUE scores.

Results on FMA-UE (Table 15) showed no significant difference in the score change after treatment between conventional therapy and RehaARM therapy. Conventional therapy and RehaARM had strong effect sizes ( $r=0.58$ and 0.57 , respectively). As a result, the samples sizes needed to show the effectiveness of the treatment were similar for conventional therapy $(n=27$; power $=0.8)$ and RehaARM therapy $(n=28$; power $=0.8)$.

Results on FIM (Table 15) showed no significant difference in the score change after treatment between conventional therapy and RehaARM therapy. Conventional therapy and RehaARM had strong effect sizes ( $r=0.6$ and 0.57 , respectively). In addition, the samples sizes needed to show treatment effectiveness were similar for 
Table 15 effect of therapy on the FMA-UE and FIM scale in the RehaARM and CVT group

\begin{tabular}{|c|c|c|c|c|}
\hline & & Conv Therapy (n=19) & RehaARM (n=19) & $\mathbf{p}$ \\
\hline Age & & $62.4(66.4 ; 53.1)$ & $52(68.3 ; 44)$ & $0.36^{\mathrm{a}}$ \\
\hline $\operatorname{Sex}(M / F)$ & & $14 / 5(74 \% / 26 \%)$ & 12/7(63\% / 37\%) & $0.49^{b}$ \\
\hline SRI & $\begin{array}{c}\text { median(Q3;Q1) } \\
\text { n; } \leq 3 \text { months } \\
\text { n; } 3<\text { months }<12 \\
\mathrm{n} ; \geq 12 \text { months }\end{array}$ & $\begin{array}{c}5.1(10.4 ; 3) \\
5(26 \%) \\
10(53 \%) \\
4(21 \%)\end{array}$ & $\begin{array}{c}5.9(8.7 ; 3.1) \\
4(21)^{\mathrm{b}} \\
12(63) \\
3(16)\end{array}$ & $0.8^{\mathrm{b}}$ \\
\hline Stroke type & Ischemic & $\begin{array}{c}13(68 \%) \\
6(32 \%)\end{array}$ & $\begin{array}{c}13(68 \%) \\
6(32 \%)\end{array}$ & $1^{\mathrm{b}}$ \\
\hline FMA & $\begin{array}{c}\operatorname{median}(\mathrm{Q} 3 ; \mathrm{Q} 1) \\
\mathrm{n} ;<=38 \\
\mathrm{n} ;>38\end{array}$ & $\begin{array}{c}16(44 ; 6) \\
14(74 \%) \\
5(26 \%)\end{array}$ & $\begin{array}{c}12(42 ; 8) \\
14(74) \\
5(26)\end{array}$ & $1^{\mathrm{b}}$ \\
\hline Acc. to FMA & $\begin{array}{c}\text { Median } \Delta \text { (Post-Pre) } \\
\text { Effect size r; p-value } \\
\text { Sample size (power=0.8) }\end{array}$ & $\begin{array}{c}4 \\
0.58 ; \mathrm{p}^{<0.001^{\mathrm{c}}} \\
27\end{array}$ & $\begin{array}{c}3 \\
0.57 ; \mathrm{p}^{<0.001^{\mathrm{c}}} \\
28\end{array}$ & $0.79^{a}$ \\
\hline Acc. to FIM & $\begin{array}{c}\text { Median } \Delta \text { (Post-Pre) } \\
\text { Effect size r; p-value } \\
\text { Sample size (power=0.8) }\end{array}$ & $\begin{array}{c}5 \\
0.6 ; p^{<0.001}{ }^{c} \\
25\end{array}$ & $\begin{array}{c}4 \\
0.57 ; p^{<0.001^{c}} \\
28\end{array}$ & $0.8^{\mathrm{a}}$ \\
\hline $\mathrm{N}$ over MCID & $\begin{array}{c}\text { FMA-UE } \\
\text { FIM }\end{array}$ & $\begin{array}{c}8(47 \%) \\
1(5 \%)\end{array}$ & $\begin{array}{l}6(32 \%) \\
3(16 \%)\end{array}$ & \\
\hline
\end{tabular}

$0.8)$.

Similar numbers of patients in both groups achieved the clinically meaningful change (MCID) in the FMA-UE score: 8 of the 19 patients (42\%) assigned to the CVT and 6 of the 19 patients (32\%) assigned to RehaARM therapy improved by at least five points. Furthermore, similar numbers of patients in both groups achieved the clinically meaningful change in the FIM score: 1 of the 19 patients (5\%) assigned to the RehaARM therapy and 3 of the 19 patients (16\%) assigned to RehaARM therapy improved by at least 22 points, Table 15 .

\subsubsection{Discussion}

The results of this study agree with existing evidence that arm robotic-assisted therapy improves body functions and may be beneficial for improving ADLs [5]. Of the patients treated with the RehaARM, 35\% achieved the clinically meaningful change in FMA-UE score and the effect size of the therapy was strong $(r=0.54$; 
$\mathrm{p}<0.001)$. The FIM score also increased significantly after treatment, with a strong effect size $(r=0.55 ; p<0.001)$. However, the 3 out of $20(15 \%)$ patients reaching the MCID was lower than for FMA-UE.

According to the FMA-UE scale, there is strong evidence that the RehaARM treatment was more effective for the subacute and mild population as well as the $\mathrm{PACl}$ group, in terms of large effect sizes and motor function improvements above the established MCID. The FMA-UE score increased for the POCI group above the MCID, but the sample size was too low $(n=2)$ to be able to draw any conclusions for this group.

According to the FIM scale, there is strong statistical evidence that the treatment was more effective for severe and subacute patients, TACl and PACl group in terms of large effect sizes.

Of severe-to-moderate patients 3 out of $14(21 \%)$ reached the MCID on the FMA-UE scale. Of mild patients a higher percentage, 4 out of 6 patients (67\%), reached the MCID. A higher training dose and dosage of RehaARM robotic-assisted therapy may be necessary for more severely impaired patients. Future clinical trials should aim at determining these quantities. It has been suggested that durable and intense robotic therapy ( 5 days a week of 5 h/day for 12 weeks) leads to an incremental progression and seems to be necessary for patients with severe-to-moderate impairment as shown in the study by Daly and colleagues using the Inmotion shoulder-Elbow robot (the 12 involved severe-to-moderate patients were also chronic, [19]).

The effect of the treatment on EMG-S was larger than on TORQUE-S for all patients and subgroups (group A and group B) likely due to subjects adapting their motor control to and training with myoelectric control. Additionally, myoelectric control allowed subjects to train with less physical and mental fatigue (larger CR and more 
positive ratings in questionnaires for myoelectric than torque control; see section 3.2.4). Nonetheless, the neurological results, based on torque and myoelectric control, revealed muscle activation improvement after treatment.

Correlation analysis revealed that the neurophysiological measures have a significant relationship with the FMA-UE, but not the FIM scale. This strong, positive correlation is a very encouraging result. The FMA-UE is grounded in well-defined, observable stages of motor recovery and is taken as the gold standard against which the validity of other scales is assessed [45]. EMG-S and TORQUE-S can be used to tailor a treatment and infer motor impairment changes.

The improvement in strength of the rotator cuff muscles significantly increased for the severe-to-moderate group A for the tasks ADD and FE. This is a very important result given the 2:1 ratio scapulohumeral rhythm, i.e. the contribution of the scapula muscles to the arm movements (see also section 3.1.2.2). For example, for $90^{\circ}$ flexion, the scapular muscles become active and move in the setting phase, which is the preparatory phase which spans the first 30 degrees of the movement.

No significant improvements were found for the trigger muscles for any of the groups.

Therapists reported that observation of the EMG signals during training was very useful because observing the relative activation of muscles during task execution allowed them to instruct the patient better. The neurophysiological measures can be used to assess abnormal activation patterns and adjust the treatment according to individual needs.

Training with the RehaARM robot combined with conventional therapy improved both motor function and independence in ADLs equally well as conventional therapy alone at a comparable dose and dosage. 
There was no statistical difference between the RehaARM and CVT conditions according to the FMA-UE and FIM scores. Similar numbers of patients in both groups achieved the clinically meaningful change in FMA-UE and FIM scores. Results agree with the available clinical evidence that same dose-matched exercise with robots and conventional therapy are equally effective [8], [26], [34], [76].

Pilot clinical studies and systematic reviews have suggested that one advantage of robot-assisted therapies may be an increase in repetitions during arm training due to a greater motivation to train [17]. Our findings suggest that the increased number of repetitions with the robotic therapy in comparison to conventional therapy within a comparable unit of time did not improve the clinical outcomes. This does not rule out that performance may increase along with training time. The robot is capable of tracking these changes accurately. Furthermore, the conventional therapy group had 20 sessions while the RehaARM therapy group had 17 sessions. The non-significant higher effect of conventional therapy alone - one point higher for both FMA-UE and FIM scales - demonstrates that these 3 additional sessions did not lead to better outcomes for the conventional therapy group.

Robotic devices allow patients to train more independently since patients are engaged in a virtual stimulating environment performing functional tasks at their own pace ([17], [77]). The RehaARM robotic-assisted therapy also permitted patients to train more independently using the robot as a vehicle. Meanwhile, the physiotherapist was able to read and document on the computer the patient's report while supervising the patient. Patients were especially motivated with the RehaARM therapy when they saw that the muscle activation surpassed the threshold more often with training. The RehaARM therapy allows patients with varying impairment levels to train shoulder movements safely and enjoyably. 
The required patient preparation for the RehaARM therapy was short (approx. 5-10 min). Therefore, this therapy can be used as an additional component within therapeutic concepts, such as in an efficiency-focused setting where one therapist treats two $0^{5}$ or more patients [19] or two therapists treat three or more patients with similar impairments. In this way, more high-intensive therapy can be offered with the same amount of personnel. Alternatively, robotic therapy can complement the upper limb therapy in a setting where conventional therapy is carried out by a physiotherapy or occupational therapist and the robotic therapy is performed by an apprentice of either field since the procedure to work with the robot can be simplified to a greater degree, compared with the procedures of conventional therapy. For example, on the first therapy day the therapist adjusts the initial robot settings and the therapy plan and the instructed apprentice executes the following therapy sessions. Supervision should be an important element in order for the apprentice to learn how to adjust the robot if needed. I have developed this upper limb training concept for the therapy assistants together with the head of occupational therapy at my current job in the Rehaklinik Zihlschlacht using the Armeo Spring from Hocoma. The dosage of upper limb therapy has been increased and we can welcome in the clinic young apprentices for their internship period. They learn about the use of robots in neurorehabilitation in combination with conventional therapy. The results are very positive so far. Furthermore, robotic therapy could be offered via telerehabilitation in a home environment for outpatients where the physiotherapist continuous offering therapy remotely to patients who are suitable for the intervention and were instructed on how to use the robot in the inpatient-period. This development has the potential of saving travelling time and incrementing the independent time of training. For the RehaARM therapy a patient's relative can be 
instructed on how to place the electrodes. As the myoelectric interface can be transferred to other robotic applications, the system and therefore the placing of the electrodes could be simplified (e.g. a portable system for the shoulder with less electrodes for assisting shoulder flexion and extension movements which are necessary for reaching tasks).

Some limitations are present in the current study with the RehaARM. This trial is still small in sample size. Therefore, the sample sizes for the subgroups were insufficient to perform statistical tests in some cases - due to low power - and detect significant differences in the primary outcomes (e.g. the subacute, chronic POCl groups). Further clinical trials with the RehaARM therapy with myoelectric control should determine with sufficiently high statistical power to which extent various patient populations profit from the treatment. The results of this study can guide decisions for future research (e.g. as a basis for selection of sub-groups, and power and sample calculations). Another limitation is the inability of the system to adjust the assistance; therefore, arm strength training may have been restricted. Further development on the RehaARM system should aim to automatically adapt this parameter as subjects improve. Alternatively, this parameter could be adaptable online by the therapist. 


\section{Conclusions}

Currently commercially available force sensors and robotic devices are unable to detect the forces/movements of many severely disabled patients. Many of them are still capable of generating residual voluntary EMG, which can be used as the control signal for the rehabilitation robot. This allows those patients to engage in early-stage user-driven rehabilitation training.

Study 1 showed that myoelectric control of the RehaARM robot with thresholding, using multichannel bipolar EMG measurements and single-DoF shoulder tasks, was feasible in a clinical setting. Stroke patients with varying levels of motor impairment were able to use the system safely and without problems. That more severely impaired patients were able to use the system is particularly remarkable. As this means that robotic-assisted therapy becomes another viable treatment option for this stroke population.

Study 2 demonstrated that patients are capable of controlling the RehaARM using interaction forces (torque control). However, the sensitivity and transparency - and by extension the robotic device's responsiveness - depend on the technical characteristics of the robotic device. Myoelectric signals are sensitive control inputs that are generated and measured independently of the robot. Study 2 showed that more severely impaired patients were able to control the assistive RehaARM system more easily via myoelectric signals than via torque control, i.e., with less physical and mental fatigue.

Study 3 showed that the EMG-driven RehaARM in combination with conventional treatment offers an effective treatment alternative for upper limb therapy. Patients of 
all levels of motor impairment engaged in the therapy and trained independently with the therapist's guidance. Robotic systems, such as the RehaARM and associated therapeutic concept proposed in this PhD project, offer new possibilities for efficient therapy with a lower than one ratio of therapists to patients ${ }^{5}$. This could allow for the provision of more intensive therapy and reduction of healthcare costs. Furthermore, the presented neurophysiological and kinematic measurements complement the clinical measurements and can guide and support the physiotherapist in tailoring the therapy to the individual, assessing the level of improvement during therapy and adapting the therapy to that improvement.

This project's scientific and clinical outcomes are the foundation of the scientific achievements in robot-aided rehabilitation within the MYOSENS project. The Fondazione Ospedale San Camillo continues using the RehaARM robot with myoelectric control for upper limb treatment after stroke in its clinical routine. Furthermore, Hocoma ${ }^{10}$ and I are currently exploring the possibility of applying the findings of this $\mathrm{PhD}$ project into a commercially available robotic application. This underlines the continuing impact of the work presented on the field of roboticassisted therapies for upper limb recovery after stroke. This PhD project - through testing the efficacy of upper limb therapy after stroke in studies with stroke patients and a clinical trial - contribute to the advancement of robotic-assisted upper limb therapy.

\footnotetext{
${ }^{10}$ Hocoma is the global market leader for the development, manufacturing and marketing of robotic and sensor-based devices for functional movement therapy (https://www.hocoma.com/world/en/about-us/company/)
} 


\section{$\underline{\text { References }}$}

[1] L. Paredes, S. Dosen, C. Genna, D. Farina, and A. Turolla, "A Novel Pneumatic EMG-driven Robotic System for Shoulder Rehabilitation after Stroke," Submitt. to JNER, BioMed Cent.

[2] L. P. Paredes, D. Farina, Y. Shin, and A. Turolla, "Efficacy of torque versus myocontrol for active, robotic-assisted rehabilitation of the shoulder after stroke: An experimental study," in 2015 7th International IEEE/EMBS Conference on Neural Engineering (NER), 2015, pp. 627-630.

[3] G. A. Donnan, M. Fisher, M. Macleod, and S. M. Davis, "Stroke," The Lancet, vol. 371, no. 9624. pp. 1612-1623, May-2008.

[4] A. G. Thrift, H. M. Dewey, R. a L. Macdonell, J. J. Mcneil, and G. a Donnan, "Incidence of the Major Stroke Subtypes," Popul. (English Ed., pp. 1732-1738, 2001

[5] J. Mehrholz, M. Pohl, T. Platz, J. Kugler, and B. Elsner, "Electromechanical and robot-assisted arm training for improving activities of daily living, arm function, and arm muscle strength after stroke.," Cochrane database Syst. Rev., vol. 11, no. 6, p. CD006876, 2015.

[6] H. S. Jørgensen, H. Nakayama, H. O. Raaschou, and T. S. Olsen, "Stroke. Neurologic and functional recovery the Copenhagen Stroke Study.," Phys. Med. Rehabil. Clin. N. Am., vol. 10, no. 4, pp. 887-906, Nov. 1999.

[7] G. Kwakkel, B. J. Kollen, J. V. Van der Grond, and A. J. H. Prevo, "Probability of regaining dexterity in the flaccid upper limb: Impact of severity of paresis and time since onset in acute stroke," Stroke, vol. 34, no. 9, pp. 2181-2186, 
2003.

[8] C. J. Winstein, J. Stein, R. Arena, B. Bates, L. R. Cherney, S. C. Cramer, F. Deruyter, J. J. Eng, B. Fisher, R. L. Harvey, C. E. Lang, M. Mackay-lyons, K. J. Ottenbacher, S. Pugh, M. J. Reeves, L. G. Richards, L. Otr, W. Stiers, and A. Rp, AHA / ASA Guideline Guidelines for Adult Stroke Rehabilitation and Recovery. 2016.

[9] J. Liepert, H. Bauder, H. R. Wolfgang, W. H. Miltner, E. Taub, and C. Weiller, "Treatment-induced cortical reorganization after stroke in humans.," Stroke., vol. 31, no. 6, pp. 1210-1216, 2000.

[10] G. Nelles, "Cortical reorganization--effects of intensive therapy," Restor. Neurol. Neurosci., vol. 22, no. 3-5, pp. 239-244, 2004.

[11] J. D. Schaechter, "Motor rehabilitation and brain plasticity after hemiparetic stroke," Prog. Neurobiol., vol. 73, no. 1, pp. 61-72, 2004.

[12] C. E. Levy, D. S. Nichols, P. M. Schmalbrock, P. Keller, and D. W. Chakeres, "Functional $\{\mathrm{MRI}\}$ evidence of cortical reorganization in upper-limb stroke hemiplegia treated with constraint-induced movement therapy," Am. J. Phys. Med. Rehabil., vol. 80, no. 1, pp. 4-12, 2001.

[13] N. A. Bayona, J. Bitensky, K. Salter, and R. Teasell, "The role of task-specific training in rehabilitation therapies.," Top. Stroke Rehabil., vol. 12, no. 3, pp. 58-65, 2005.

[14] I. J. Hubbard, M. W. Parsons, C. Neilson, and L. M. Carey, "Task-specific training: evidence for and translation to clinical practice.," Occup. Ther. Int., vol. 16, no. 3-4, pp. 175-89, Jan. 2009.

[15] D. Corbetta, V. Sirtori, G. Castellini, L. Moja, and R. Gatti, "Constraint-induced movement therapy for upper extremities in people with stroke.," Cochrane 
database Syst. Rev., vol. 10, no. 10, p. CD004433, 2015.

[16] L. Ke, S. George, S. Thomas, D. Je, and M. Crotty, "Virtual reality for stroke rehabilitation ( Review )," no. 9, 2011.

[17] G. Kwakkel, B. J. Kollen, and H. I. Krebs, "Effects of robot-assisted therapy on upper limb recovery after stroke: a systematic review.," Neurorehabil. Neural Repair, vol. 22, no. 2, pp. 111-21, 2008.

[18] C. Ochoa Luna, M. H. Rahman, M. Saad, P. S. Archambault, and S. B. Ferrer, "Admittance-based Upper Limb Robotic Active and Active-assistive Movements," Int. J. Adv. Robot. Syst., 2015.

[19] J. J. Daly, N. Hogan, E. M. Perepezko, H. I. Krebs, J. M. Rogers, K. S. Goyal, M. E. Dohring, E. Fredrickson, J. Nethery, and R. L. Ruff, "Response to upperlimb robotics and functional neuromuscular," J. Rehabil. Res. Dev., vol. 42, no. 6, p. $723,2005$.

[20] D. G. Caldwell and N. Tsagarakis, "Biomimetic actuators in prosthetic and rehabilitation applications.," Technol. Health Care, vol. 10, no. 2, pp. 107-20, Jan. 2002.

[21] N. Jarrassé, T. Proietti, V. Crocher, J. Robertson, A. Sahbani, G. Morel, and A. Roby-Brami, "Robotic Exoskeletons: A Perspective for the Rehabilitation of Arm Coordination in Stroke Patients," Front. Hum. Neurosci., vol. 8, no. December, p. 947, 2014.

[22] R. Morales, F. J. Badesa, N. García-Aracil, J. M. Sabater, and C. Pérez-Vidal, "Pneumatic robotic systems for upper limb rehabilitation.," Med. Biol. Eng. Comput., vol. 49, no. 10, pp. 1145-56, Oct. 2011.

[23] H. I. Krebs, N. Hogan, M. L. Aisen, and B. T. Volpe, "Robot-aided neurorehabilitation.," IEEE Trans. Rehabil. Eng., vol. 6, no. 1, pp. 75-87, Mar. 
1998.

[24] G. Kwakkel, B. J. Kollen, and H. I. Krebs, "Effects of robot-assisted therapy on upper limb recovery after stroke: a systematic review.," Neurorehabil. Neural Repair, vol. 22, no. 2, pp. 111-21, 2009.

[25] P. S. Lum, C. G. Burgar, M. Van der Loos, P. C. Shor, M. Majmundar, and R. Yap, "MIME robotic device for upper-limb neurorehabilitation in subacute stroke subjects: A follow-up study.," J. Rehabil. Res. Dev., vol. 43, no. 5, pp. $631-42$.

[26] V. Klamroth-Marganska, J. Blanco, K. Campen, A. Curt, V. Dietz, T. Ettlin, M. Felder, B. Fellinghauer, M. Guidali, A. Kollmar, A. Luft, T. Nef, C. SchusterAmft, W. Stahel, and R. Riener, "Three-dimensional, task-specific robot therapy of the arm after stroke: a multicentre, parallel-group randomised trial.," Lancet Neurol., vol. 13, no. 2, pp. 159-66, Feb. 2014.

[27] P. Culmer, A. Jackson, R. Richardson, B. Bhakta, M. Levesley, and A. Cozens, "Development of a dual robotic system for upper-limb stroke rehabilitation," Proc. 2005 IEEE 9th Int. Conf. Rehabil. Robot., vol. 2005, pp. 61-65, 2005.

[28] S. Balasubramanian, M. Perez, B. Shepard, E. Koeneman, and J. Koeneman, "RUPERT: An exoskeleton robot for assisting rehabilitation of arm functions," in 2008 Virtual Rehabilitation, 2008, pp. 163-167.

[29] R. J. Sanchez, E. Wolbrecht, R. Smith, J. Liu, S. Rao, S. Cramer, T. Rahman, J. E. Bobrow, D. J. Reinkensmeyer, and P. Shah, "A pneumatic robot for retraining arm movement after stroke: Rationale and mechanical design," in Proceedings of the 2005 IEEE 9th International Conference on Rehabilitation Robotics, 2005, vol. 2005, pp. 500-504. 
[30] J. Stein, K. Narendran, J. McBean, K. Krebs, and R. Hughes, "Electromyography-controlled exoskeletal upper-limb-powered orthosis for exercise training after stroke.," Am. J. Phys. Med. Rehabil., vol. 86, no. 4, pp. 255-261, Apr. 2007.

[31] P. S. Lum, C. G. Burgar, P. C. Shor, M. Majmundar, and M. Van der Loos, "Robot-assisted movement training compared with conventional therapy techniques for the rehabilitation of upper-limb motor function after stroke," Arch. Phys. Med. Rehabil., vol. 83, no. 7, pp. 952-959, Jul. 2002.

[32] H. Vallery, M. Guidali, A. Duschau-Wicke, and R. Riener, "Patient-cooperative control: Providing safe support without restricting movement," IFMBE Proc., vol. 25, no. 9, pp. 166-169, 2009.

[33] E. Colgate and N. Hogan, "An analysis of contact instability in terms of passive physical equivalents," Proceedings, 1989 Int. Conf. Robot. Autom., pp. 404409, 1989.

[34] G. Kwakkel and C. G. M. Meskers, "Effects of robotic therapy of the arm after stroke.," Lancet Neurol., vol. 13, no. 2, pp. 132-3, Feb. 2014.

[35] S. Lee and Y. Sankai, "Power assist control for leg with HAL-3 based on virtual torque and impedance adjustment," IEEE Int. Conf. Syst. Man Cybern., vol. 4, no. October, pp. 1499-1504, 2002.

[36] J. Rosen, M. Brand, M. B. Fuchs, and M. Arcan, "A myosignal-based powered exoskeleton system," IEEE Trans. Syst. Man, Cybern. - Part A Syst. Humans, vol. 31, no. 3, pp. 210-222, May 2001.

[37] X. Hu, K. Y. Tong, R. Song, V. S. Tsang, P. O. Leung, and L. Li, "Variation of Muscle Coactivation Patterns in Chronic Stroke During Robot-Assisted Elbow Training," Arch. Phys. Med. Rehabil., vol. 88, no. 8, pp. 1022-1029, 2007. 
[38] X. L. Hu, K.-Y. Tong, R. Song, X. J. Zheng, and W. W. F. Leung, "A comparison between electromyography-driven robot and passive motion device on wrist rehabilitation for chronic stroke.," Neurorehabil. Neural Repair, vol. 23, no. 8, pp. 837-46, Oct. 2009.

[39] F. Daerden and D. Lefeber, "Pneumatic Artificial Muscles: actuators for robotics and automation."

[40] S. Raine and M. Linzi, Bobath Concept, 1 Edition. Oxford, UK: WileyBlackwell, 2009.

[41] R. Paine and M. L. Voight, "The role of the scapula.," Int. J. Sports Phys. Ther., vol. 8, no. 5, pp. 617-29, 2013.

[42] V. C. K. Cheung, L. Piron, M. Agostini, S. Silvoni, A. Turolla, and E. Bizzi, "Stability of muscle synergies for voluntary actions after cortical stroke in humans.," Proc. Natl. Acad. Sci. U. S. A., vol. 106, no. 46, pp. 19563-8, Nov. 2009.

[43] H. J. Hermens, B. Freriks, C. Disselhorst-Klug, and G. Rau, "Development of recommendations for SEMG sensors and sensor placement procedures.," J. Electromyogr. Kinesiol., vol. 10, no. 5, pp. 361-74, Oct. 2000.

[44] A. R. Fugl-Meyer, L. Jääskö, I. Leyman, S. Olsson, and S. Steglind, "The poststroke hemiplegic patient. 1. a method for evaluation of physical performance.," Scand. J. Rehabil. Med., vol. 7, no. 1, pp. 13-31, 1975.

[45] D. J. Gladstone, C. J. Danells, and S. E. Black, "The fugl-meyer assessment of motor recovery after stroke: a critical review of its measurement properties.," Neurorehabil. Neural Repair, vol. 16, no. 3, pp. 232-240, 2002.

[46] R. Salter, Katherine; Campbell, Nerissa; Richardson, Marina; Mehta, Swati; Jutai, Jeffrey; Zettler, Laura; Moses, Matthew; McClure, Andrew; Mays, 
Rachel; Foley, Norine; Teasell, "Outcome measures in stroke rehabilitation," Evidence-Based Rev. Stroke Rehabil., vol. 110, pp. 1-144, 2013.

[47] S. J. Page, G. D. Fulk, and P. Boyne, "Clinically important differences for the upper-extremity Fugl-Meyer Scale in people with minimal to moderate impairment due to chronic stroke.," Phys. Ther., vol. 92, no. 6, pp. 791-8, 2012.

[48] B. Ashworth, "Preliminary trial of carisoprodol in multiplo sclerosis," Practitioner, vol. 192, pp. 540-2, Apr. 1964.

[49] R. W. Bohannon and M. B. Smith, "Interrater Reliability of a Modified Ashworth Scale of Muscle Spasticity," Phys. Ther., vol. 67, no. 2, pp. 206-207, Feb. 1987.

[50] M. F. Levin, J. Desrosiers, D. Beauchemin, N. Bergeron, and A. Rochette, "Development and validation of a scale for rating motor compensations used for reaching in patients with hemiparesis: the reaching performance scale.," Phys. Ther., vol. 84, no. 1, pp. 8-22, Jan. 2004.

[51] R. Keith, "The functional independence measure: a new tool for rehabilitation.," Adv. Clin. Rehabil., vol. 1, pp. 6-18, 1987.

[52] M. Beninato, K. M. Gill-Body, S. Salles, P. C. Stark, R. M. Black-Schaffer, and J. Stein, "Determination of the minimal clinically important difference in the FIM instrument in patients with stroke," Arch. Phys. Med. Rehabil., vol. 87, no. 1, pp. 32-39, 2006.

[53] M. Kellor, J. Frost, N. Silberberg, I. Iversen, and R. Cummings, "Hand strength and dexterity.," Am. J. Occup. Ther., vol. 25, no. 2, pp. 77-83, Mar. 1971.

[54] V. Mathiowetz, G. Volland, N. Kashman, and K. Weber, "Adult norms for the Box and Block Test of manual dexterity.," The American journal of 
occupational therapy.: official publication of the American Occupational Therapy Association, vol. 39, no. 6. pp. 386-391, 1985.

[55] E. Bizzi, A. D'Avella, P. Saltiel, and M. Tresch, "Modular Organization of Spinal Motor Systems," Neuroscientist, vol. 8, no. 5, pp. 437-442, 2002.

[56] A. d'Avella, P. Saltiel, and E. Bizzi, "Combinations of muscle synergies in the construction of a natural motor behavior.," Nat. Neurosci., vol. 6, no. 3, pp. 300-8, Mar. 2003.

[57] D. D. Lee, B. Laboratories, M. Hill, and H. S. S. Ý, "Algorithms for Nonnegative Matrix Factorization," no. 1.

[58] A. Turolla, M. Dam, L. Ventura, P. Tonin, M. Agostini, C. Zucconi, P. Kiper, A. Cagnin, and L. Piron, "Virtual reality for the rehabilitation of the upper limb motor function after stroke: a prospective controlled trial.," J. Neuroeng. Rehabil., vol. 10, no. 1, p. 85, Jan. 2013.

[59] R. . T. Floyd and C. Thompson, Manual of Structural Kinesiology. McGraw-Hill Humanities/Social Sciences/Languages, 2011.

[60] N. Takeuchi and S.-I. Izumi, "Rehabilitation with poststroke motor recovery: a review with a focus on neural plasticity.," Stroke Res. Treat., vol. 2013, p. 128641, Jan. 2013.

[61] S. J. Housman, L. Otr, K. M. Scott, and D. J. Reinkensmeyer, "Hemiparesis," pp. 505-514, 2009.

[62] P. Langhorne, J. Bernhardt, and G. Kwakkel, "Stroke rehabilitation.," Lancet, vol. 377, no. 9778, pp. 1693-702, May 2011.

[63] R. Pfeifer, M. Lungarella, and F. lida, "Self- Organization, Embodiment, and Biologically Inspired Robotics," Science (80-. )., vol. 318, no. 5853, pp. 10881093, 2007. 
[64] B. Cesqui, P. Tropea, S. Micera, and H. I. Krebs, "EMG-based pattern recognition approach in post stroke robot-aided rehabilitation: a feasibility study.," J. Neuroeng. Rehabil., vol. 10, no. 1, p. 75, Jul. 2013.

[65] D. J. Reinkensmeyer, C. D. Takahashi, W. K. Timoszyk, A. N. Reinkensmeyer, and L. E. Kahn, "Design of robot assistance for arm movement therapy following stroke," Adv. Robot., vol. 14, no. 7, pp. 625-637, Jan. 2001.

[66] R. F. Beer, M. D. Ellis, B. G. Holubar, and J. P. A. Dewald, "Impact of gravity loading on post-stroke reaching and its relationship to weakness," Muscle and Nerve, vol. 36, no. 2, pp. 242-250, 2007.

[67] A. Ameri, E. Scheme, E. Kamavuako, K. Englehart, and P. Parker, "Real Time, Simultaneous Myoelectric Control Using Force and Position Based Training Paradigms.," IEEE Trans Biomed Eng, vol. 61, no. 2, pp. 279-287, 2014.

[68] T. E. TWITCHELL, "The restoration of motor function following hemiplegia in man.," Brain, vol. 74, no. 4, pp. 443-80, Dec. 1951.

[69] J. Loscalzo, "Pilot Trials in Clinical Research Of What Value Are They ?," pp. 1694-1697, 2004.

[70] F. R. Pezzella, O. Picconi, A. De Luca, P. D. Lyden, and M. Fiorelli, "Development of the Italian version of the national institutes of health stroke scale it-NIHSS," Stroke, vol. 40, no. 7, pp. 2557-2559, 2009.

[71] J. Bamford, P. Sandercock, M. Dennis, C. Warlow, and J. Burn, "Classification and natural history of clinically identifiable subtypes of cerebral infarction," Lancet, vol. 337, no. 8756, pp. 1521-1526, 1991.

[72] P. M. Davies, Steps to Follow, 2nd ed. Berlin, Heidelberg: Springer Berlin Heidelberg, 2000.

[73] J. Cohen, Statistical power analysis for the behavioral sciences, 2nd ed., vol. 
2nd. 2013.

[74] J. Pallant, SPSS survival manual, 3rd ed., vol. 36, no. 3. 2007.

[75] B. Rosner, Fundamentals of Biostatistics, 7th ed. Boston, MA: Wadsworth Publishing, 2011.

[76] A. C. Lo, P. D. Guarino, L. G. Richards, J. K. Haselkorn, G. F. Wittenberg, D. G. Federman, R. J. Ringer, T. H. Wagner, H. I. Krebs, B. T. Volpe, C. T. Bever, D. M. Bravata, P. W. Duncan, B. H. Corn, A. D. Maffucci, S. E. Nadeau, S. S. Conroy, J. M. Powell, G. D. Huang, and P. Peduzzi, "Robot-assisted therapy for long-term upper-limb impairment after stroke," N. Engl. J. Med., vol. 362, no. 19, pp. 1772-1783, 2010.

[77] G. B. Prange, M. J. A. Jannink, A. H. A. Stienen, H. van der Kooij, M. J. IJzerman, and H. J. Hermens, "Influence of Gravity Compensation on Muscle Activation Patterns During Different Temporal Phases of Arm Movements of Stroke Patients," Neurorehabil. Neural Repair, vol. 23, no. 5, pp. 478-485, 2009.

[78] C. Genna, S. Dosen, L. Paredes, A. Turolla, B. Graimann, and D. Farina, "A Novel Robot-Aided Therapy for Shoulder Rehabilitation after Stroke: ActiveAssisted Control of the RehaArm Robot Using Electromyographic Signals," in 2nd International Conference on NeuroRehabilitation, ICNR, 2014, pp. 383391. 


\section{Annex I: List of disseminations}

As part of this PhD, I have submitted one article about study 1 to BioMed Central (JNER) and contributed to a conference paper. In addition, I presented two posters, poster 1 in $7^{\text {th }}$ International IEEE EMBS conference, Montpellier and poster 2 in the $8^{\text {th }}$ World Congress of Neurorehabilitation WCNR, Istanbul in order to share studies 1 and 2 with the community. Finally, the manuscript on the clinical trial is currently in preparation.

\section{Conferences/Symposia}

\begin{tabular}{|l|l|}
\hline Poster 1 & $\begin{array}{l}\text { This poster has been published as conference paper and it showed } \\
\text { preliminary results of study 2. }\end{array}$ \\
& $\begin{array}{l}\text { L. P. Paredes, D. Farina, S. Member, Y. Shin, and A. Turolla, } \\
\text { "Efficacy of Torque versus Myocontrol for Active, Robotic - } \\
\text { Assisted Rehabilitation of the Shoulder after Stroke : An } \\
\text { Experimental Study," 7th Annu. Int. IEEE EMBS Conf. Neural } \\
\text { Eng., pp. 22-24, 201[2]. }\end{array}$ \\
\hline Poster 2 & $\begin{array}{l}\text { L. P. Paredes, C. Genna, M.L. Bullo, S. Dosen, D. Cattin, D. Farina } \\
\text { and A. Turolla, "Torque and Myoelectric control for Robotic- } \\
\text { Assisted Rehabilitation of the shoulder after stroke: a Clinical and } \\
\text { Experimental Study". }\end{array}$ \\
\hline
\end{tabular}

\section{Journal and conference Publications}

\begin{tabular}{|l|l|}
\hline Submitted & $\begin{array}{r}\text { L. Paredes, S. Dosen, C. Genna, D. Farina, and A. Turolla, "A Novel } \\
\text { Pneumatic EMG-driven Robotic System for Shoulder } \\
\text { Rehabilitation after Stroke," Submitt. to JNER, BioMed Cent.[1]. }\end{array}$ \\
\hline $\begin{array}{l}\text { In } \\
\text { preparation }\end{array}$ & $\begin{array}{r}\text { L. Paredes, S. Dosen, G. Pregnolato, A. Turolla and D. Farina } \\
\text { "Myoelectric Robot-Assisted Rehabilitation for the Upper Limb after } \\
\text { Stroke: a phase II clinical trial," In prep. }\end{array}$ \\
\hline Co-author & $\begin{array}{l}\text { C. Genna, S. Dosen, L. Paredes, A. Turolla, B. Graimann, and D. } \\
\text { Farina, "A Novel Robot-Aided Therapy for Shoulder Rehabilitation } \\
\text { after Stroke: Active-Assisted Control of the RehaArm Robot Using } \\
\text { Electromyographic Signals," in 2nd International Conference on } \\
\text { NeuroRehabilitation, ICNR 2014, pp. 383-391 [78]. }\end{array}$ \\
\hline
\end{tabular}




\section{Annex II: Curriculum Vitae}

LILIANA PATRICIA PAREDES CALDERÓN
Dipl.-Ing.
$\begin{array}{ll}\text { Persönliche } & \text { Nationalität: Kolumbien } \\ \text { Angaben: } & \begin{array}{l}\text { Geburtsdatum: } 18.04 .1985 \\ \text { Ledig }\end{array} \\ \text { Postanschrift: } & \begin{array}{l}\text { Arbonerstrasse } 35 \\ \\ \end{array} \\ & \text { S590 Romanshorn } \\ & \text { Schweiz }\end{array}$

Kontakt: $\quad$ +41-(0)-762811985

liliana.paredes1985@gmail.com

Berufserfahrung

04/2016- Rehaklinik Zihlschlacht, Zihlschlacht, Schweiz

heute Leiterin Robotik

06/2015- Otto Bock Healthcare GmbH, Duderstadt, Deutschland

01/2016 Entwicklungsingenieurin für Neurorehabilitation Engineering und Prothetik.

06/2013- Fondazione Ospedale San Camillo - I.R.C.C.S., Lido di Venezia, Italien

05/2015 Arbeits- und Forschungsaufenthalt an einer anerkannten Rehabilitationsklinik mit Fokus auf neurodegenerative Krankheiten und Schlaganfall im Rahmen eines europäischen Förderprojektes. Zu meinen Aufgaben zählt die Implementierung und Evaluierung von klinischen Studien zum Einsatz von Robotern für die Rehabilitation von Schlaganfällen. Die Ergebnisse dieser Arbeit werden in meine Dissertation an der Universitätsmedizin Göttingen einfließen.

10/2011- Otto Bock Healthcare GmbH, Duderstadt, Deutschland

05/2013 Entwicklungsingenieurin für Neurorehabilitation Engineering und Prothetik. U.a. habe ich an der Entwicklung neuer Mensch-Maschine-Schnittstellen für Handprothesen mitgewirkt und die Ergebnisse in Probandenstudien mit Amputierten evaluiert. Im Rahmen des EU-IAPP Projektes Myosens wurde ich im Juni 2013 nach Italien an das Fondazione Ospedale San Camillo entsendet.

10/2009- Technische Universität Wien, Wien, Österreich

04/2011 Wissenschaftliche Hilfskraft unter der Betreuung von Professor Dipl. Ing, DDDr. Frank Rattay im Fachbereich Computational Neuroscience, Institute of Analysis and Scientific Computing.

Ausbildung

seit Universitätsmedizin Göttingen, Georg-August Universität Göttingen, 10/2013 Deutschland

Promotionsstudiengang Humanwissenschaften in der Medizin zur robotergestützten Rehabilitation von Schlaganfallpatienten. Die formalen Anforderungen habe ich bereits erfüllt und erwarte die Promotion im Jahr 2015 abzuschließen.

10/2009- Technische Universität Wien, Wien, Österreich

06/2011 Masterstudiengang Biomedical Engineering (überwiegend deutschsprachig), Abschluss 
mit Auszeichnung bestanden.

2004- Universidad Nacional de Colombia, Bogota, Kolumbien

2009 Bachelorstudiengang Elektrotechnik mit Vertiefungsfächern in digitaler Signalverarbeitung sowie Steuerungs- und Automatisierungstechnik.

2002- John Bapst Memorial High School, Maine, USA

2003 Schüleraustausch im letzten Jahr der Schulausbildung zur Erreichung des USamerikanischen High-School-Abschlusses.

1996- El Carmen Teresiano High school, Cúcuta, Kolumbien

2003 Erwerb der Hochschulzugangsberechtigung für ein Studium in Kolumbien.

Lehre \& Fachvorträge

11/2015 Bobath-Grundkurs Erwachsene

Theorie und Praxis, 2. Woche: Posturale Kontrolle, Gleichgewicht, Stabilität, Transfers, Aktivitäten der oberen Extremitäten, Neurophysiologie und Neuropathophysiologie, Befund und Behandlung von verschiedenen Krankheitsbildern, Workshops mit Patienten

11/2014- Dozentin im BSc. Orthobionik, PFH Private Hochschule Göttingen, Deutschland

01/2015 Vorlesung Neuroprothetik: Mensch-Maschine-Schnittstellen, Reha-Roboter und Cochlea Implantate

04/2014 World Congress of Neurorehabilitation, Istanbul, Türkei

Posterpräsentation: Torque and Myoelectric Control for Robotic-Assisted Rehabilitation of the Shoulder after Stroke: A Clinical And Experimental Study

03/2013 Technically Assisted Rehabilitation (TAR) Conference, Berlin, Deutschland Vortrag: Towards Robustness in Pattern Recognition based Myoelectric Prosthesis Control

10/2010- Technische Universität Wien, Wien, Österreich

02/2011 Tutorin für den Masterkurs "Computer Simulation in Medicine".

02/2007- Universidad Nacional de Colombia, Bogota, Kolumbien

10/2008 Tutorin für den Kurs "Analogue Electronics I and III" an der Elektrotechnischen Fakultät.

Sonstige Kenntnisse

\begin{tabular}{lll}
\hline Sprachen & - & Spanisch (Muttersprache) \\
& - & Englsch (Verhandlungssicher) \\
& - Italienisch (Verhandlungssicher) \\
PC & - Microsoft Windows \\
Kenntnisse & - Microsoft Office, Tex/Latex/Beamer \\
& - Adobe Creative Suite (Illustrator, Photoshop, Premiere) \\
& - MATLAB, Labview, C/C++
\end{tabular}

Weiterbildung

Otto Bock Academy Seminar "Einstieg in die Service Excellence für Ottobock Guides“

Otto Bock Academy Seminar Produkterlebnistag "ich will's wissen“

Otto Bock Academy Seminar „Lernen Lernen“

Veröffentlichungen

L. P. Paredes, S. Dosen, C. Genna, D. Farina, A. Turolla, "A Novel Pneumatic EMG-driven Robotic System for Shoulder Rehabilitation after Stroke" ( Submitt. to JNER, BioMed Cent)

L. P. Paredes, S. Dosen, F. Rattay, B. Graimann, and D. Farina, "The impact of the stimulation 
frequency on closed-loop control with electrotactile feedback," J. Neuroeng. Rehabil., Dec. 2015. S. Amsüss, P. M. Goebel, N. Jiang, B. Graimann, L. Paredes, and D. Farina, "Self-correcting pattern recognition system of surface EMG signals for upper limb prosthesis control.," IEEE Trans. Biomed. Eng., vol. 61, no. 4, pp. 1167-76, Apr. 2014.

M. M. Vidovic, L. P. Paredes, H.-J. Hwang, S. Amsuess, J. Pahl, J. M. Hahne, B. Graimann, D. Farina, and K.-R. Mueller, "Covariate Shift Adaptation in EMG Pattern Recognition for Prosthetic Device Control," Conf. Proc. Annu. Int. Conf. IEEE Eng. Med. Biol. Soc., 2014.

S. Amsuss, L. P. Paredes, N. Rudigkeit, B. Graimann, M. J. Herrmann, and D. Farina, "Long term stability of surface EMG pattern classification for prosthetic control.," Conf. Proc. IEEE Eng. Med. Biol. Soc., vol. 2013, pp. 3622-3625, Jul. 2013.

L. P. Paredes and B. Graimann, "Advanced Myoelectric Control of Prostheses: Requirements and Challenges," in Converging clinical \& engineering research on NR, vol. 1, 2013, pp. 1221-1224.

N. Ge, P. M. Goebel, S. Amsuess, L. Paredes, R. Pawlik, and D. Farina, "Evaluating Upper-Limb EMGProsthesis User Performance by Combining Psychometric Measures and Classification-Rates *," 6th Int. IEEE EMBS Conf. Neural Eng., pp. 4-7, 2013.

H. Rehbaum, N. Jiang, L. Paredes, S. Amsuess, B. Graimann, and D. Farina, "Real time simultaneous and proportional control of multiple degrees of freedom from surface EMG: Preliminary results on subjects with limb deficiency.," Conf. Proc. IEEE Eng. Med. Biol. Soc., vol. 2012, pp. 1346-9, Jan. 2012.

F. Rattay, L. P. Paredes, and R. N. Leao, "Strength-duration relationship for intra- versus extracellular stimulation with microelectrodes.," Neuroscience, vol. 214, pp. 1-13, Jul. 2012.

C. Wenger, L. Paredes, and F. Rattay, "Current-Distance Relations for Microelectrode Stimulation of Pyramidal Cells," Artif. Organs, vol. 35, no. 3, pp. 263-266, Mar. 2011.

L.P. Paredes, C. Wenger, F. Rattay, "Enhancement of vowel encoding for cochlear implants by adding a high frequency signal: a modelling study", Proc. 10th Vienna International Workshop on Functional Electrical Stimulation, 2010.

Persönliche Interessen

Sport und Bewegung sind für mich der Ausgleich nach der täglichen Arbeit. Daher genieße ich es verschiedene Sportarten zu betrieben, wie z.B. Joggen, Tanzen, Inline-Skaten, Radfahren und Volleyball.

Fremde Sprachen und Kulturen sind meine Leidenschaft, daher genieße ich es zu reisen, neue Orte und Menschen kennen zu lernen, um dadurch andere Kulturen zu erkunden. 